\title{
The CALOCUBE project for a space based cosmic ray experiment: design, construction, and first performance of a high granularity calorimeter prototype.
}

\author{
O. Adriani, ${ }^{a, b}$ S. Albergo, ${ }^{c, d}$ L. Auditore, ${ }^{e, d}$ A. Basti, ${ }^{f, g}$ E. Berti, ${ }^{a, b}$ G. Bigongiari, ${ }^{h, f}$ \\ L. Bonechi, ${ }^{b}$ M. Bongi, ${ }^{a, b}$ V. Bonvicini, ${ }^{i}$ S. Bottai, ${ }^{b}$ P. Brogi, ${ }^{h, f}$ G. Cappello, ${ }^{c, d}$ \\ G. Carotenuto, ${ }^{j}$ G. Castellini, ${ }^{k, b}$ P.W. Cattaneo, ${ }^{l}$ R. Cecchi, ${ }^{h, f}$ C. Checchia, ${ }^{m, n}$ \\ R. D’Alessandro, ${ }^{a, b, 1}$ S. Detti, ${ }^{b}$ M. Fasoli, ${ }^{o, p}$ N. Finetti, ${ }^{q, b}$ A. Italiano, ${ }^{d}$ P. Lenzi, ${ }^{a, b}$ \\ M.G. Pellegriti, ${ }^{d}$ P. Maestro, ${ }^{h, f}$ M. Manetti, ${ }^{b}$ P.S. Marrocchesi, ${ }^{h, f}$ N. Mori, ${ }^{b}$ F. Morsani, ${ }^{f}$ \\ M. Olmi, ${ }^{b}$ A. Orsini, ${ }^{f}$ G. Orzan, ${ }^{i}$ L. Pacini, ${ }^{a} b$ P. Papini, ${ }^{b}$ A. Rappoldi, ${ }^{l}$ S. Ricciarini, ${ }^{k}, b$ \\ A. Sciuto, ${ }^{r}$ P. Spillantini, ${ }^{b}$ O. Starodubtsev, ${ }^{b}$ L. Stiaccini, ${ }^{h, f}$ F. Stolzi, ${ }^{h, f}$ A. Sulaj, ${ }^{h, f}$
}

J.E Suh, ${ }^{h, f}$ A. Tiberio, ${ }^{a, b}$ A. Tricomi, ${ }^{c, d}$ A. Trifirò, ${ }^{e, d}$ M. Trimarchi, ${ }^{e, d}$ E. Vannuccini, ${ }^{b}$

A. Vedda, ${ }^{o, p}$ G. Zampa, ${ }^{i}$ N. Zampa ${ }^{i}$

${ }^{a}$ Department of Physics and Astronomy, University of Florence, Via G. Sansone 1, I-50019 Sesto Fiorentino
(Firenze), Italy
${ }^{b}$ INFN Sezione di Firenze, Via B. Rossi 1, I-50019 Sesto Fiorentino (Firenze), Italy
${ }^{c}$ Department of Physics and Astronomy, University of Catania, Via S. Sofia 64, I-95123 Catania, Italy
${ }^{d} I N F N$ Sezione di Catania, Via S. Sofia 64, I-95123 Catania, Italy
${ }^{e}$ MIFT, University of Messina, Viale F. Stagno d'Alcontres 31, I-98166 Messina, Italy
${ }^{f}$ INFN Sezione di Pisa, Largo Bruno Pontecorvo 3, I-56127 Pisa, Italy
${ }^{g}$ Department of Physics, University of Pisa, Largo Bruno Pontecorvo 3, I-56127 Pisa, Italy
${ }^{h}$ Department of Physical Sciences, Earth and Environment, University of Siena, I-53100 Siena, Italy
${ }^{i} I N F N$ Sezione di Trieste, Padriciano 99, I-34149 Trieste, Italy
${ }^{j}$ IPCB-CNR, P.le Enrico Fermi 1, Portici, I-80055 Napoli, Italy
${ }^{k}$ IFAC-CNR,Via Madonna del Piano 10, I-50019 Sesto Fiorentino (Firenze), Italy
${ }^{l}$ INFN Sezione di Pavia, Via Agostino Bassi 6, I-27100 Pavia, Italy
${ }^{m}$ Department of Physics and Astronomy, University of Padova, Via F. Marzolo 8, I-35131 Padova, Italy
${ }^{n}$ INFN Sezione di Padova, Via Francesco Marzolo 8, I-35121 Padova, Italy
${ }^{o}$ Department of Materials Science, University of Milano-Bicocca, via Cozzi 55, I-20125 Milan, Italy
${ }^{p}$ INFN Sezione di Milano-Bicocca, Piazza della Scienza, 3, I-20126 Milano, Italy
${ }^{q}$ Department of Physics and Chemistry, University of L'Aquila, Via Vetoio 40, I-67100 L'Aquila, Italy
${ }^{r}$ IMM-CNR, Str. VIII Zona Industriale, I-95121 Catania, Italy
E-mail: candi@fi .infn. it

${ }^{1}$ Corresponding author. 
Aвstract: Current research in High Energy Cosmic Ray Physics touches on fundamental questions regarding the origin of cosmic rays, their composition, the acceleration mechanisms, and their production. Unambiguous measurements of the energy spectra and of the composition of cosmic rays at the "knee" region could provide some of the answers to the above questions. So far only ground based observations, which rely on sophisticated models describing high energy interactions in the earth's atmosphere, have been possible due to the extremely low particle rates at these energies.

A calorimetry based space experiment that could provide not only flux measurements but also energy spectra and particle identification, would certainly overcome some of the uncertainties of ground based experiments. Given the expected particle fluxes, a very large acceptance is needed to collect a sufficient quantity of data, in a time compatible with the duration of a space mission. This in turn, contrasts with the lightness and compactness requirements for space based experiments.

We present a novel idea in calorimetry which addresses these issues whilst limiting the mass and volume of the detector. In this paper we report on a four year R\&D program where we investigated materials, coatings, photo-sensors, Front End electronics, and mechanical structures with the aim of designing a high performance, high granularity calorimeter with the largest possible acceptance. Details are given of the design choices, component characterisation, and of the construction of a sizeable prototype (Calocube) which has been used in various tests with particle beams.

KEYwords: Calorimeters, Space instrumentation, Scintillators 


\section{Contents}

1 Introduction and detector concept $\quad 1$

2 Photodiode and scintillator choice 3

2.1 Introduction 3

2.2 Photodiodes 4

$\begin{array}{lll}2.3 & \text { Photodiode single channel readout and calibration } & 6\end{array}$

2.3.1 Optimization and calibration of the set-up $\quad 7$

2.3.2 Absolute charge calibration procedure $\quad 8$

$\begin{array}{lll}2.4 & \text { Scintillating crystal choice and characterisation } & 10\end{array}$

$\begin{array}{lll}2.5 & \text { Full sized crystal calibration } & 14\end{array}$

$\begin{array}{lll}2.6 & \text { Light collection efficiency } & 15\end{array}$

$\begin{array}{lll}2.6 .1 & \text { Simulation } & 15\end{array}$

$\begin{array}{lll}\text { 2.6.2 Measurements } & 17\end{array}$

$\begin{array}{lll}2.7 & \mathrm{CsI}(\mathrm{Tl}) \text { crystal response with cosmic rays } & 20\end{array}$

3 Calorimeter mechanical design studies 22

3.1 Carbon fibre tray prototypes and development 22

$\begin{array}{lll}3.2 & \text { Carbon fibre tray measurements results } & 24\end{array}$

$\begin{array}{ll}3.3 & \text { Calorimeter test beam prototype } \\ \end{array}$

4 Front End electronics and final Test Beam prototype 31

4.1 The CASIS Front End Electronics 31

4.2 The calorimeter assemblies 33

4.3 Prototype Assembly and Readout 36

4.4 Current and final development 39

4.5 Dual readout studies 40

5 Conclusions $\quad 42$

\section{Introduction and detector concept}

Protons and nuclei energy spectra from cosmic rays, show a "knee" structure at around $1 \mathrm{PeV}$ (see figure 1, taken from [9]), which could signal a change of regime due to various possible causes, i.e. production mechanisms, an upper energy limit of galactic accelerators, an energy dependant cosmic ray composition, the presence of cutoffs in the transportation of cosmic rays. Spectral measurements in the knee region are derived from data collected by ground-based shower detectors [1-8] whose reliability would benefit greatly if direct, above the earth's atmosphere, measurements in the PeV region were to be made available. As shown in figure 1, such a measurement faces enormous 
challenges due to the extremely low flux of expected particles at those energies (1 particle per $\mathrm{m}^{2}$ per year). Thus an orbiting detector must provide not only a very good energy resolution for hadrons $(<40 \%)$ and a way to identify the cosmic ray atomic number, but must also have a large acceptance (few $\mathrm{m}^{2} \mathrm{sr}$ ). This last requirement is in direct antithesis to the constraints of space missions where volume and mass are at premium [10]. The design we present in this paper has a high granularity

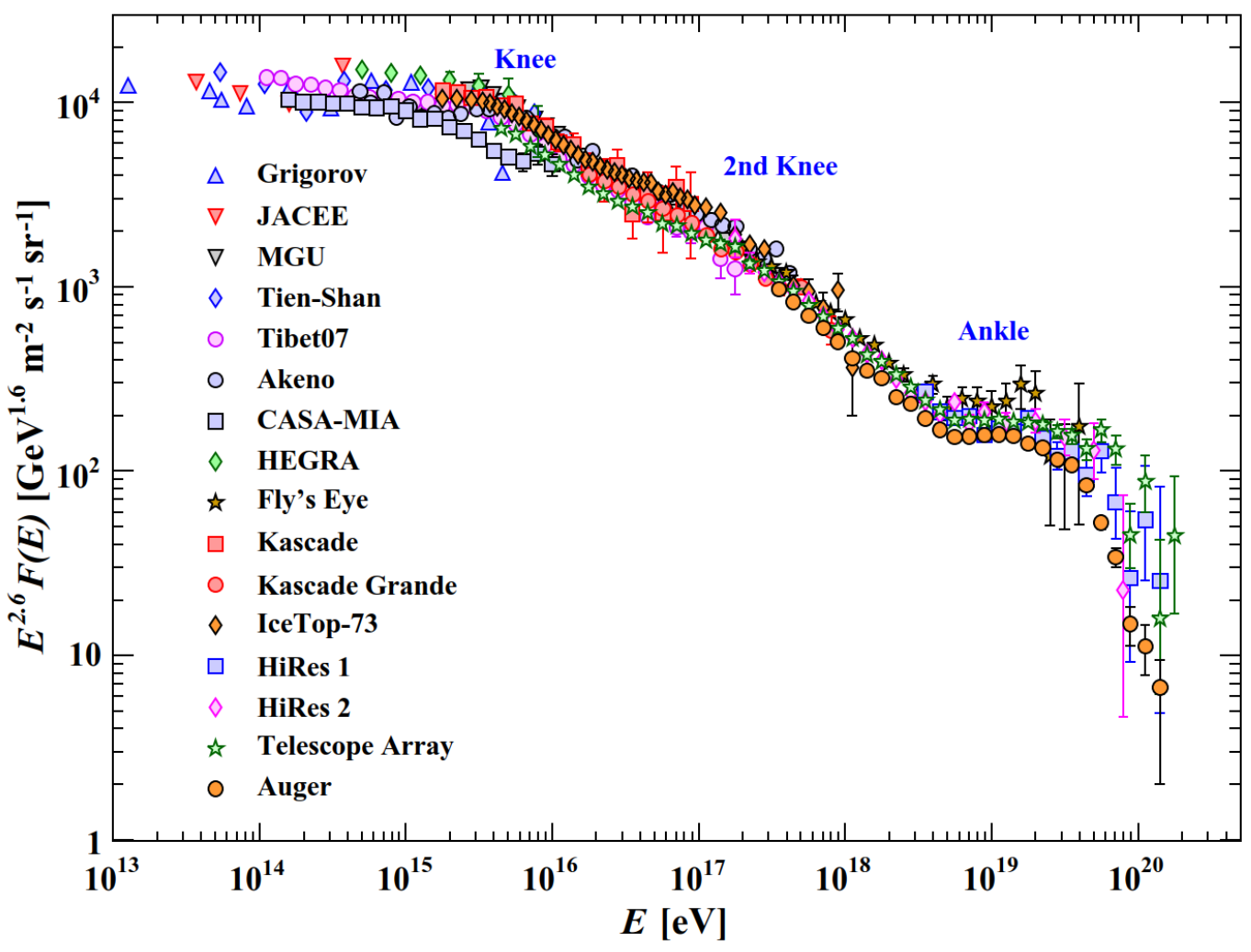

Figure 1. Cosmic ray energy spectrum. In evidence the slope changes (knee, ankle) and expected particle fluxes .

with a homogeneous segmentation both laterally and depthwise. This unique design allows to achieve an excellent energy resolution using shower shape reconstruction algorithms, an enhanced distinction between hadrons and electrons, and a large acceptance obtained by maximising the number of entrance windows to the detector thus keeping a reasonable mass and volume budget (1.6 tons, $<1 \mathrm{~m}^{3}$ ). In order to faithfully reconstruct the most energetic showers while maintaining sensitivity to Minimum Ionizing Particles (MIPs), a dynamic range on the order of $10^{7}$ is required. This has been achieved with a dual photodiode readout and a novel Front End electronics design (see following sections).

From the start, we have proceeded with a calorimeter design optimised for space applications. Thus volume and mass constraints have always been considered of paramount importance (i.e. acceptance increases should rely only on novel approaches that do not necessarily involve a volume increase). The fundamental tenet of the design is a cubic detector in which 5 of the 6 sides are capable of particle detection and measurement in a totally symmetric and interchangeable way. Such a design effectively translates in a five fold acceptance increase while keeping the overall 
volume constant.

Mass constraints, on the other hand, translate in a maximum number of interaction lengths $\left(\lambda_{\mathrm{I}}\right)$ which, depending on the material used, can be at most 2-3. A high granularity approach to the design, allows us to reconstruct a detailed image of each interacting particle shower profile. This not only helps us achieve a high discrimination between electrons and protons/nuclei, but also a relatively high energy resolution for hadrons using partial shower profile fits, given that for obvious limits, hadronic showers will not be fully contained.
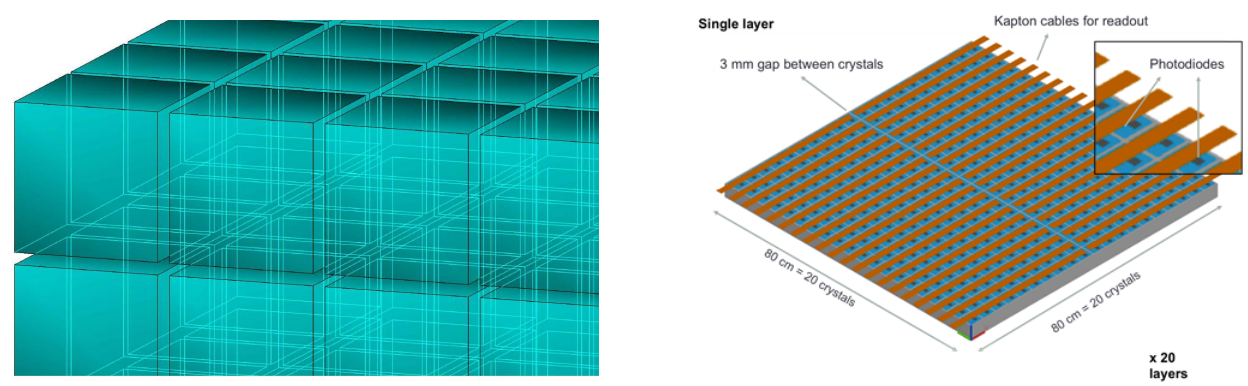

Figure 2. Conceptual mesh design for the Calocube prototype (left). Drawing of a tray holding together a matrix of 20x20 crystals (right). Also depicted the Kapton PCBs bringing out the photodiodes signals.

Conceptually the Calocube calorimeter consists of a mesh of cubic scintillator crystals (see figure 2) arranged as a stack of trays similar to the one depicted in the figure holding together a matrix of small crystals, each small cube having an edge of roughly one Moliere radius. The total number of radiation lengths $\left(\mathrm{X}_{0}\right)$ is of the order of 40 with a corresponding number of $\lambda_{\mathrm{I}}$ between 1 and 2 depending on the final design depth and scintillator material choice. The gaps between each crystal are kept as constant as possible to avoid any non homogenous detector response. Not only are the crystals packed as closely as possible but also the trays.

We have realised a large size prototype (using roughly 700 cubic crystals), with which we have taken data using particle and ion beams at CERN. During its construction, various choices on detector material, mechanical structure, readout electronics, and diode sensors were investigated and validated with the aim of a final design submission for a full scale detector to be flown on the chinese space station (HERD Mission [11]). Translating this design concept into a viable project that could be prototyped has not been an easy passage. To this end, our collaboration has investigated many solutions. We have performed detailed mechanical simulations on a number of design choices [12], we have characterised various scintillator materials, evaluated readout solutions for the scintillators, developed custom chips for the Front End electronics, and constructed a $\sim 700$ crystal protoype that has been tested with particle beams. The following sections will detail these aspects of our R\&D with the results obtained, highlighting the ensuing design choices.

\section{Photodiode and scintillator choice}

\subsection{Introduction}

We based our scintillator choice mainly on the obtainable electromagnetic energy resolution, but also considering both the radiation and interaction lengths of the material. In fact, the need to provide 
even partial hadronic shower containment, while at the same time keeping mass, volume and costs at a reasonable scale, narrows down the available choices. To improve the energy resolution for hadronic showers, we also considered Cherenkov light detection in the same crystal, not unlike the DREAM [13] project at CERN. Unfortunately while we have presented results [14] [15] showing an improvement in the hadronic energy resolution, we have found that this becomes significant only in the case of full shower containment which is not our case. We will show some tests we performed in section 4.5.

As described in the following sections, we performed several studies and tests on inorganic scintillators which we considered as plausible candidates for the Calocube calorimeter. Consequently we also tested the light collection efficiency for various wrapping schemes both reflective and diffusive. We used commercial photodiodes (PDs) as photosensors (see sub-section below). Our single crystal tests relied on a high accuracy spectrometer by Amptek [16], consisting of the charge-sensitive preamplifier A250 coupled to the digital pulse analyser PX5. For these tests, a ${ }^{241} \mathrm{Am}$ source was used for both calibration and signal generation while some measurements were also performed with MIPS using atmospheric cosmic rays. In addition some further calibrations involved the use of LEDs at different wavelengths to illuminate directly the PDs.

\subsection{Photodiodes}
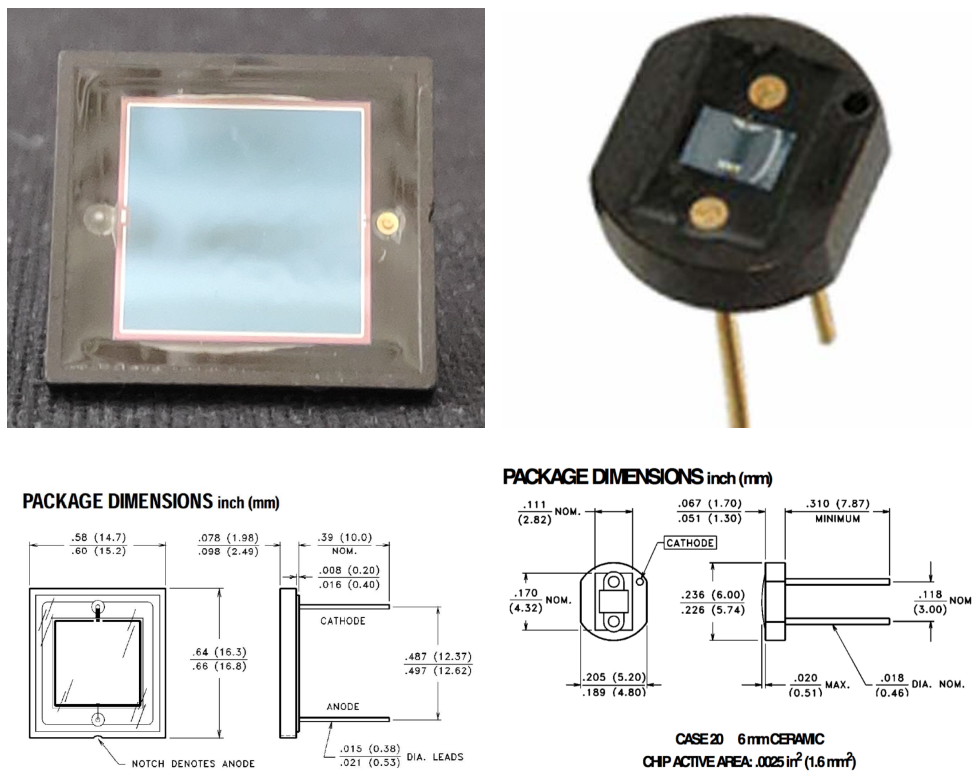

Figure 3. Excelitas VTH2090 and VTP9412 diodes with their dimensions.

There are not that many options available when choosing a photon sensor to convert the scintillator light output to electrical signals. Considering that this calorimeter should be an instrument orbiting in space, we have then stringent overall mass constraints, power requirements and dissipation, and also require compactness of the ancillary calorimeter components. We thus opted for a silicon photodiode (PD) solution, which satisfies all the above criteria and provides a very stable photon to electron conversion efficiency versus temperature and voltage biasing variations. 
Given the physics scope of the calorimeter, namely the need to correctly measure a MIP signal for calibration purposes (typically $10 \mathrm{MeV}$ per crystal), while mantaining linearity even for energy deposits of up to $100 \mathrm{TeV}$ in one crystal, we opted for a solution with two PDs each with different active area. After an extensive market analysis, we chose the following Excelitas [17] PDs: for small signals (such as those from MIPs) a large area silicon PIN photodiode VTH2090 (figure 3), and a small area VTP9412 for large signals from showers. The latter is a standard PD with an area roughly a fifty times smaller that will not saturate the F.E. electronics even in the presence of the most energetic deposits. The VTH2090 is a PIN photodiode with a $9.2 \times 9.2 \mathrm{~mm}^{2}$ active area,

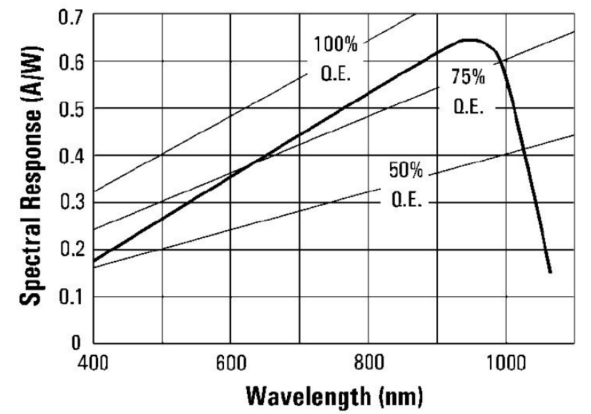

(a)

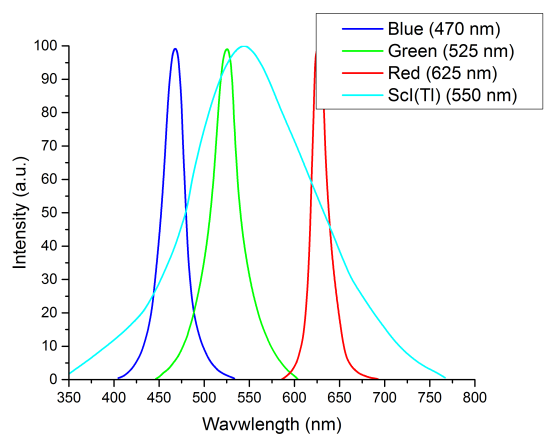

(b)

Figure 4. Spectral response of VTH2090 (VTP9412 has a similar behaviour) 4(a) and the emission spectra for the blue, green and red LEDs and the CsI(Tl) scintillator. 4(b) photodiodes.

mounted in a black ceramic package with an epoxy window. This device is ideal for scintillation detection, spectrophotometry, or other applications requiring a fast, large area, high sensitivity device. Its main features are: high quantum efficiency, excellent uniformity, high shunt impedance,

Table 1. Measured spectral responses.

\begin{tabular}{|c|c|c|}
\hline Wavelength $(\mathrm{nm})$ & VTH2090 & VTP9412 \\
\hline 470 & $0.28 \pm 0.02 \mathrm{~A} / \mathrm{W}$ & $0.33 \pm 0.02 \mathrm{~A} / \mathrm{W}$ \\
525 & $0.30 \pm 0.02 \mathrm{~A} / \mathrm{W}$ & $0.34 \pm 0.02 \mathrm{~A} / \mathrm{W}$ \\
625 & $0.38 \pm 0.03 \mathrm{~A} / \mathrm{W}$ & $0.42 \pm 0.03 \mathrm{~A} / \mathrm{W}$ \\
\hline
\end{tabular}

low junction capacitance, fast response and low noise. The VTP9412 is a fast response silicon photodiode having a $1.6 \mathrm{~mm}^{2}$ active area designed for spectral response between 400 and $1150 \mathrm{~nm}$. This series of photodiodes has been designed for low junction capacitance to achieve faster response time. These photodiodes are suitable for operation under reverse bias, which increases the speed of response, but can also be used in photovoltaic mode. Main features for this PD are: visible to IR spectral range, small active area, 1 to $2 \%$ linearity over 7 to 9 decades, low dark current, high dark resistance, low capacitance, fast response. The spectral response for both VTH2090 and VTP9412 PDs are shown in figure 4. Both are well matched to the CsI (Tl) emission spectrum also shown in the same figure, together with the emission spectra of the light sources we used for testing the PDs. We have tested both diodes with four light sources: blue, green and red LEDs, with a wavelength of 
respectively $470 \mathrm{~nm}, 525 \mathrm{~nm}, 625 \mathrm{~nm}$ and a $\mathrm{CsI}(\mathrm{Tl})$ scintillator with a spectrum maximum at 550 $\mathrm{nm}$.

The main goal of these measurements was to verify the parameters provided by the manufacturer, in particular the spectral response. Comparing the given spectral response with the measured one, we have reproduced the data shown in figure 4 within $8 \%$. Our measurements are shown in table 1.

\subsection{Photodiode single channel readout and calibration}

As stated previously, we have used a low noise DAQ system designed by Amptek for our single crystal/photodiode studies and measurements. The system consists of a charge sensitive preamplifier A250 and a digital pulse analyser PX5.

The A250 [16] is a hybrid Charge Sensitive Preamplifier for use with a wide range of detectors having capacitance from less than one, to several thousand picofarads. The input FET was selected and optimized to our specific application. Usually, this component is chosen with a large transconductance $\left(g_{m}\right)$ as possible, while matching its input capacitance $\left(C_{i s s}\right)$ to the detector capacitance $\left(C_{d}\right)$. Taking into account this fact and the PDs capacitances, we chose a parallel configuration of

Table 2. 2SK152 F.E.T. characteristics.

\begin{tabular}{|c|c|c|c|c|c|c|c|}
\hline Type & $B V_{G S S}$ & $I_{G S S}$ & $V_{G S(o f f)}$ & $I_{D S S}$ & $g_{f s}$ & $C_{i s s}$ & $C_{r s s}$ \\
\hline n-channel & -20 & 0.1 & $-0.5 /-2.0$ & $50 / 20$ & 30 & 15 & 4.0 \\
& & $(-10 \mathrm{~V})$ & $(-10 \mathrm{~V})$ & $(10 \mathrm{~V})$ & $(10 \mathrm{~V})$ & $(0-10 \mathrm{~V})$ & $(0-10 \mathrm{~V})$ \\
\hline Units & $\mathrm{V}(\mathrm{min})$ & $\mathrm{nA}(\max )$ & $\mathrm{V}(\mathrm{min} / \mathrm{max})$ & $\mathrm{mA}(\mathrm{min} / \mathrm{max})$ & $\mathrm{mS}$ & $\mathrm{pF}$ & $\mathrm{pF}$ \\
\hline
\end{tabular}

three 2SK152 FET, which has a good capacitive match with our PDs and an optimal noise performance. The 2SK152 FET characteristics are shown in table 2. Moreover, we used DC coupling between PD and A250 to exclude additional noise sources. The typical coupling circuit is shown in figure 5. The final configuration that has been used for the single channel measurements is

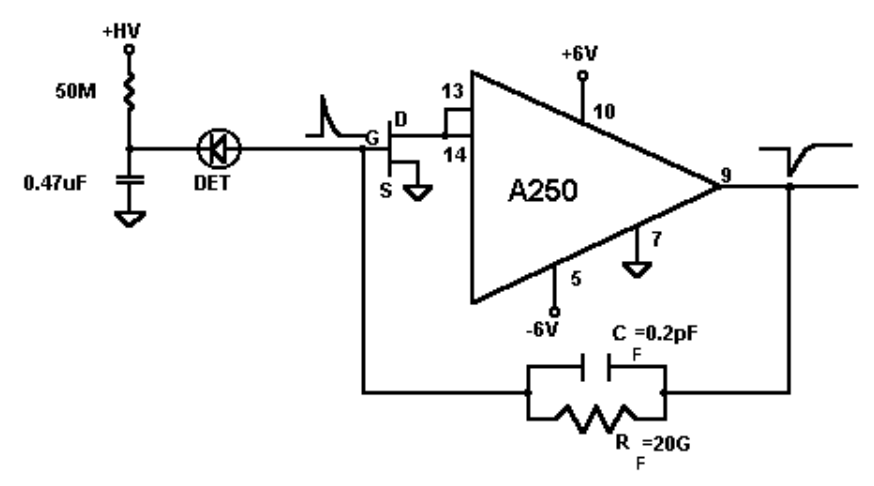

Figure 5. The A250 charge preamplifier circuit used with the DC coupling for the PD.

characterized by $3 \times 2$ SK252 as input FET, standard feedback components $\left(\mathrm{R}_{f}=300 \mathrm{M} \Omega, \mathrm{C}_{f}=1 \mathrm{pF}\right)$, DC coupled photodiode and an RC bias filter $\left(\mathrm{R}_{b}=20 \mathrm{M} \Omega, \mathrm{C}_{b}=27 \mathrm{nF}\right)$.

We used the Amptek PX5 [18] postamplifier to interface the detector (coupled to the A250 preamplifier) to a computer running data acquisition and control software. The PX5 digitizes the 


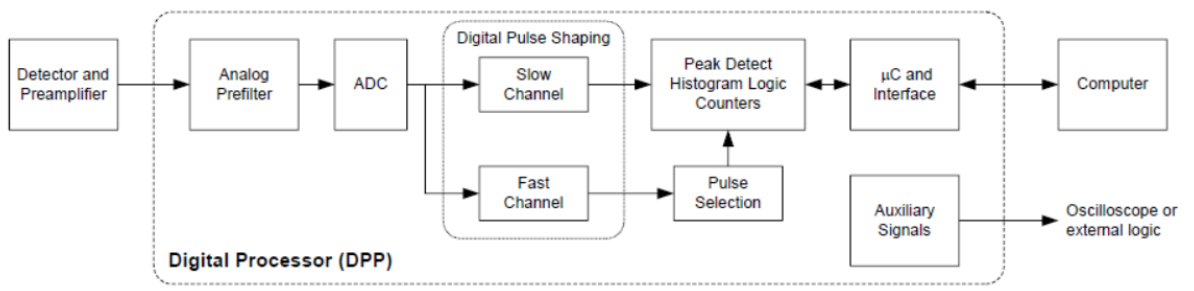

Figure 6. Block diagram of the PX5 Digital Pulse Processor (DPP) in a complete system.

preamplifier output, applies real-time digital processing to the signal, detects the peak amplitude (digitally) and bins this value in its histogramming memory, generating a signal spectrum. The Block diagram of PX5 is shown in figure 6. We used a trapezoidal pulse shaping, with a typical output pulse shape shown in figure 7. This shape provides a near optimum signal to noise ratio for many detectors. The pulse selection logic rejects pulses for which an accurate measurement cannot

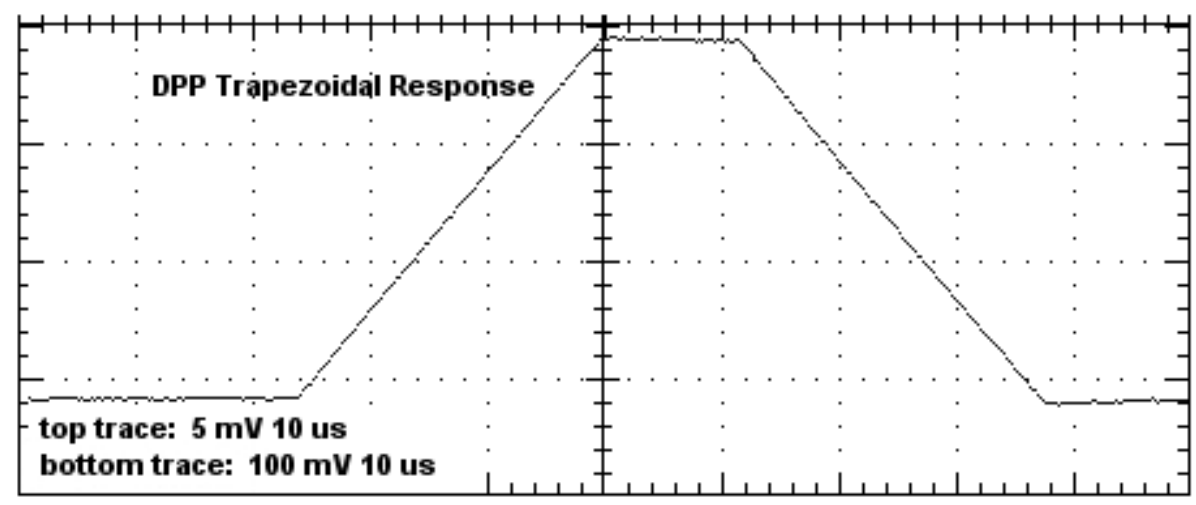

Figure 7. Trapezoidal shaped pulse of DPP.

be made. It includes pile-up rejection, rise time discrimination, logic for an external gating signal, etc. The histogram memory operates as in a traditional multichannel analyser (MCA). When a pulse occurs with a particular peak value, a counter in a corresponding memory location is incremented. The primary output of the DPP is a histogram (an array), with a bin content that follows the signal spectrum.

\subsubsection{Optimization and calibration of the set-up}

We have optimised the shaping time by performing various noise measurements [19] for the VTH2090 diode. The noise has been estimated using the Full Width Half Maximum of the pulse signal distributions (in ADC channels) for various shaping times. Noise dependence on the shaping time $\tau$ [20], is described in terms of Equivalent Noise Charge by eq. (2.1), where $C_{i n}$ is the input capacitance (sum of the detector capacitance plus the 3 FET in parallel), $R_{s}$ is the sum of the series resistance of the input FET (from source to gate), the cables arriving to the gate and the other parasitic series resistances (usually few $\Omega$ ), $R_{f}$ is the feedback resistance, $\Gamma$ is a numerical coefficient equal to $2 / 3$ for JFET, flattop is the smaller base of the trapezoid, $g_{m}$ is the transconduttance of the FET (in our case it is the sum of 3 FETs), $e_{\text {pink }}$ is the pink noise term $\left(1 / f\right.$ noise term) and $I_{\text {leak }}$ is 
the leakage current through the detector. We have fitted the experimental data using eq. (2.1). The red line present in figure 8 shows the result of the fit, with the value of $4 k T$ fixed at $0.1 \mathrm{eV}$ (room temperature) and with all other parameters free to vary within an appropriate range.

$$
E N C=\frac{1}{q} \sqrt{\left(\frac{\tau}{3}+\frac{\text { flattop }}{2}\right) \cdot\left(2 q I_{\text {leak }}+\frac{4 k T}{R_{f}}\right)+C_{i n}^{2} 4 k T\left(\frac{\Gamma}{g_{m}}+R_{s}\right) \cdot \frac{1}{\tau}+C_{i n}^{2} q e_{\text {pink }}}
$$

From the fit of figure 8 , we derive an optimal shaping time in the range $3.8 \div 4.2 \mu \mathrm{s}$. As a cross

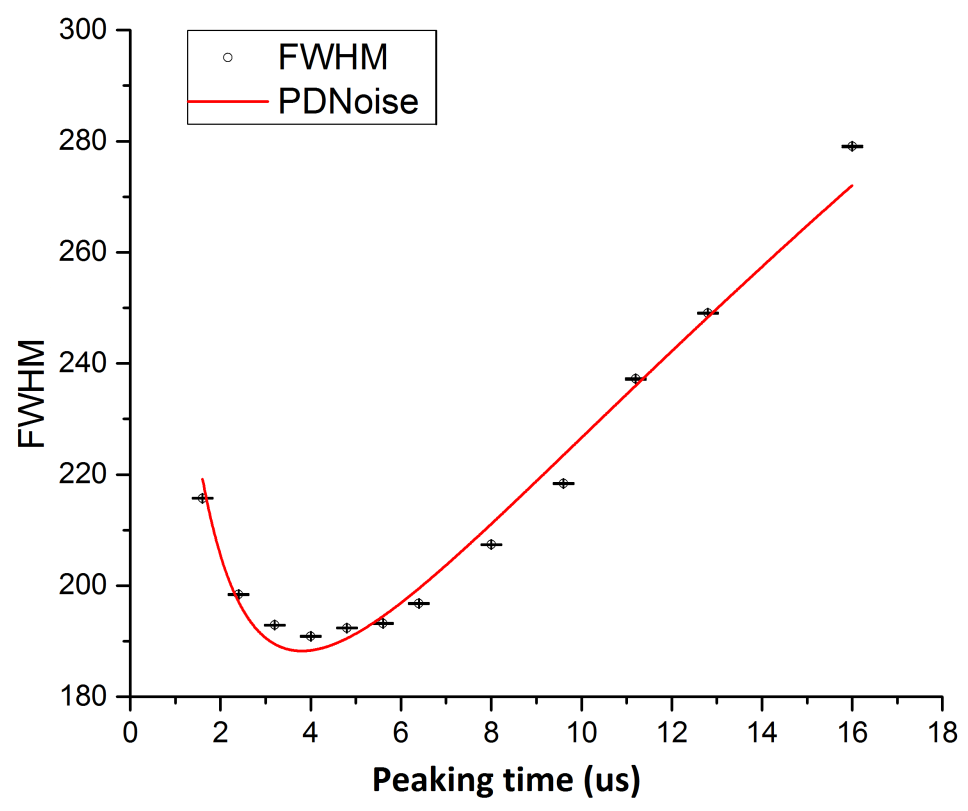

Figure 8. Noise dependence on shaping time.

check we have compared nominal and fit derived values for the free parameters as shown in table 3 .

Table 3. Parameter fit values.

\begin{tabular}{|c|c|c|}
\hline Parameter name & Nominal value & Fit value \\
\hline$C_{i n}(\mathrm{~F})$ & $1.15 \times 10^{-10}$ & $1.13 \times 10^{-10}$ \\
$R_{S}(\Omega)$ & few Ohms & 1 \\
$g_{m}(\mathrm{mS})$ & 0.09 & 0.1 \\
$R_{f}(\Omega)$ & $3 \times 10^{8}$ & $3.05 \times 10^{8}$ \\
$I_{\text {leak }}(\mathrm{A})$ & $\sim 1 \times 10^{-9}$ & $8.4 \times 10^{-10}$ \\
\hline
\end{tabular}

\subsubsection{Absolute charge calibration procedure}

To calibrate the ADC scale versus energy or charge we used a ${ }^{241} \mathrm{Am}$ source which decays into ${ }^{237} \mathrm{~Np}$ emitting a $5.5 \mathrm{MeV} \alpha$ particle. Such a source emits also $\mathrm{X}$ and $\gamma$ lines which originate mostly from decay products of ${ }^{237} \mathrm{~Np}$. We have used two of these lines ( $8 \mathrm{keV}$ and $\left.59.6 \mathrm{keV}\right)$ to calibrate 


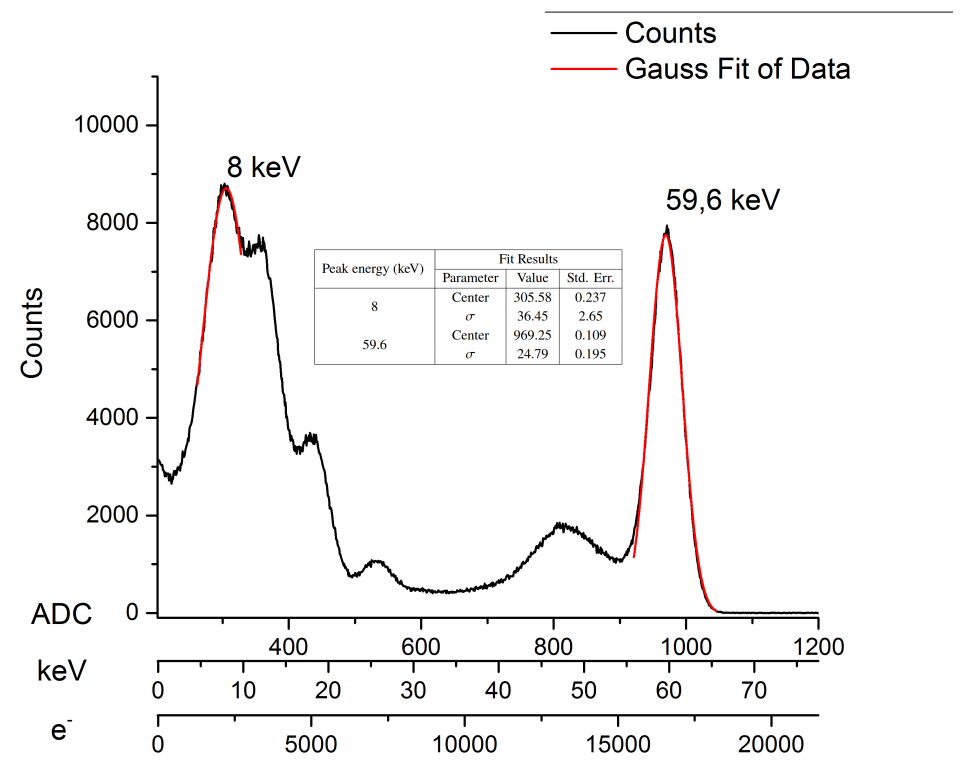

Figure 9. ${ }^{241} \mathrm{Am}$ source spectrum, with the fit results on the $8 \mathrm{keV}$ and $59.6 \mathrm{keV}$ peaks.

the system. The ${ }^{241} \mathrm{Am}$ spectrum measured directly by the VTH2090 photodiode coupled to the source, is shown in figure 9. The two calibration peaks can be easily identified and both of them have been fitted with a Gaussian function. The resulting Gaussian fits are shown with red solid lines in the figure. The energy difference between the two calibration peaks is $51.6 \mathrm{keV}$, corresponding
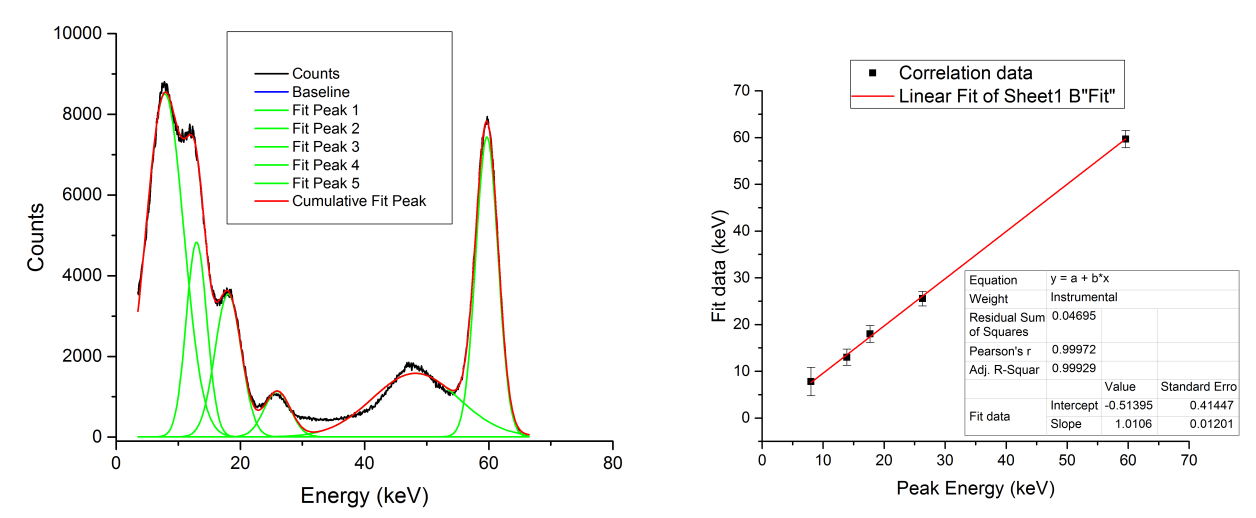

Figure 10. Multiple peak fits (left) and correlation between nominal and derived values of the ${ }^{241} \mathrm{Am}$ spectrum peaks (right).

to $663.7 \mathrm{ADC}$ channels from which we obtain a calibration factor of $77.75 \mathrm{eV}$ per ADC channel. Dividing this value by the energy needed for electron-hole pair creation in silicon, which is equal to $3.67 \mathrm{eV}$ at room temperature [21], we obtain a charge calibration factor of $21.19 \mathrm{e}^{-}$per ADC channel. Using the position of the two peaks both in energy and ADC channels, the offset can be evaluated to be about 202.7 ADC channels. In the same figure, both the energy and charge scale obtained are shown at the bottom. We have extended this procedure to the other peaks present in the ${ }^{241} \mathrm{Am}$ spectrum. The measured energy spectrum of ${ }^{241} \mathrm{Am}$ with multiple fits is shown in figure 
10 , with the result of the correlation fit using the previous scale factors to predict the peak energies. The correlation slope coefficient is 1.01 , indicating an excellent agreement when using the scale factors derived above.

\subsection{Scintillating crystal choice and characterisation}

The materials that have been considered for the Calocube detector are $\mathrm{CsI}(\mathrm{Tl})$ [22], $\mathrm{BGO}\left(\mathrm{Bi}_{4} \mathrm{Ge}_{3} \mathrm{O}_{12}\right)$ [23] and $\mathrm{LYSO}\left(\mathrm{Lu}_{2(1-x)} \mathrm{Y}_{2 x} \mathrm{SiO}_{5}(\mathrm{Ce})\right)$ [24]. All are high density materials with a small radiation

Table 4. Scintillator properties.

\begin{tabular}{|c|c|c|c|}
\hline Scintillator & CsI(Tl) & BGO & LYSO \\
\hline Density $\left(\mathrm{g} / \mathrm{cm}^{3}\right)$ & 4.51 & 7.13 & 7.30 \\
Radiation length $\mathrm{X}_{0}(\mathrm{~cm})$ & 1.85 & 1.12 & 1.16 \\
Decay time $(\mathrm{ns})$ & 1000 & 300 & 50 \\
Light yield $\left(\mathrm{e}^{-} / \mathrm{keV}_{\gamma}\right)$ & $5.5 \cdot 10^{4}$ & $\sim 8 \cdot 10^{3}$ & $\sim 2.5 \cdot 10^{4}$ \\
Wavelength of maximum emission $(\mathrm{nm})$ & 550 & 480 & 428 \\
\hline
\end{tabular}

length, capable of containing showers in a relatively small volume, a critical issue as stated before, in space applications. In particular $\mathrm{CsI}(\mathrm{Tl})$, which is a common scintillator used in nuclear and

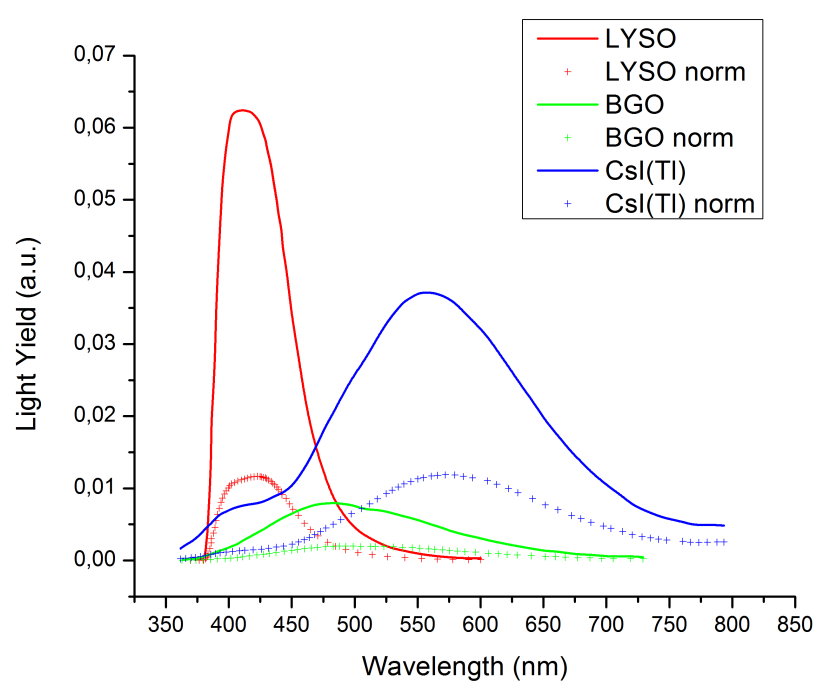

Figure 11. Scintillator emission spectra [25] and their convolution with the VTH2090 response (norm).

particle physics with a very high light yield, became our final choice for the prototype given its wide availability with many producers in the market, at an affordable price which is a very important parameter since the propotype involved the use of 1000 such crystals. Another advantage (from a space mission point of view) is that it suffers plastic deformation when stressed but is not brittle. Also the spectral response of $\mathrm{CsI}(\mathrm{Tl})$, which peaks at around $580 \mathrm{~nm}$, is very well matched to normal silicon photodiodes that are available in a wide variety of formats and electrical parameters. We summarise the main properties of our chosen crystals in table 4. 
We have found consistent discrepancies in the available literature on LYSO crystals. In fact LYSO is a relatively new material and its characteristics can change depending on the sources, this can also happen for more established scintillators such as BGO (cf. [25-27]). A comparison of the emission spectra of the three scintillators was performed by the group of Mao Rihua [25] from which the emission spectra shown in figure 11 are taken. We convoluted these with the spectral response of our photodiodes to obtain the curves shown in the same figure. The VTH2090 spectral response can be approximated by a line with offset and slope respectively equal to $-0.1779 \mathrm{~A} / \mathrm{W}$ and 8.815 . $10^{4} \mathrm{~A} / \mathrm{Wnm}$ in the range up to $800 \mathrm{~nm}$. These values have been used to convolute the scintillators emission spectra. After convolution $\mathrm{CsI}(\mathrm{Tl})$ shows the highest light yield indicating a better spectral

Table 5. Relative signal level for the scintillators.

\begin{tabular}{|c|c|c|}
\hline Scintillator & Integral value (A.U.) & \% of CsI(Tl) \\
\hline CsI(Tl) & 2.44 & 100 \\
LYSO & 0.85 & 35 \\
BGO & 0.37 & 15 \\
\hline
\end{tabular}

match to the photodiodes used. The results of the integral of the convoluted curves are presented in table 5 relative to the $\mathrm{CsI}(\mathrm{Tl})$ signal. The result is very dependent on the manufacturers data, and suffers from the data discrepancies mentioned before. These depend on the details of crystal growth, on the exact composition, and of course on the spectral response of the photon detectors used. For example according to data from "Saint-Gobain Ceramics \& Plastics, Inc." the ratio of the signals for CsI(Tl)/BGO/LYSO is 1/0.59/0.18 [22-24]. We thus performed laboratory tests of these scintillators coupled to our chosen photodiodes, to obtain the data that was used afterwards as input for the physics simulations of our calorimeter. We performed these measurements on cubic crystals

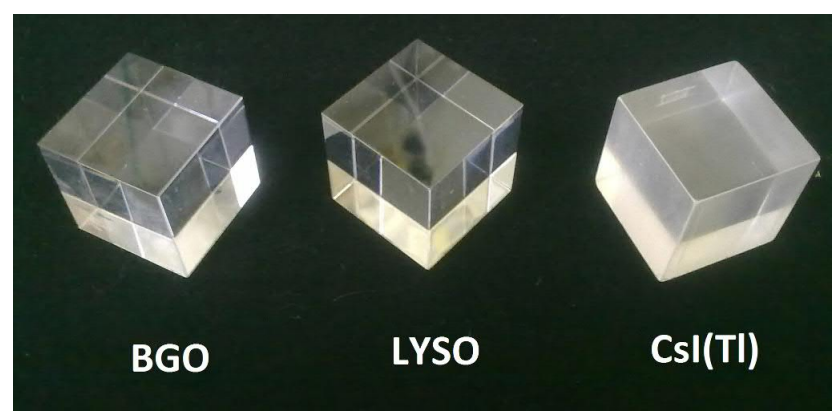

Figure 12. Cubes of CsI(Tl), BGO and LYSO with different surface finish (sanded for the CsI).

of $20 \times 20 \times 20 \mathrm{~mm}^{3}$, as shown in figure 12 . These are smaller than the dimensions needed for the prototypes but allowed us to keep the scintillator costs down without sacrificing the measurement siginficance. The crystals were wrapped with a $400 \mu \mathrm{m}$ thickness of Teflon tape. All scintillators were coupled to VTH2090 diodes. We used $\alpha$ particles from ${ }^{241}$ Am source. To minimize the external energy loss of the $\alpha$ particles in the wrapping material, a $1 \mathrm{~mm}^{2}$ window in the Teflon cover was opened and the source was placed in contact with the crystal surface. The range of $\alpha$ particle in $\mathrm{CsI}(\mathrm{Tl})$ versus energy, shown in figure 13, has been obtained using ASTAR software, developed by National Institute of Standards and Technology (NIST) [28, 29]. From the figure, a $5 \mathrm{MeV} \alpha$ 
particle has $0.054 \mathrm{~cm}$ range in $\mathrm{CsI}(\mathrm{Tl})$ (likewise for the other crystals under study), thus it is totally contained within the scintillator cubes used for the measurements and the energy deposited depends only on the $\alpha$ particle energy independently of the scintillator under examination.

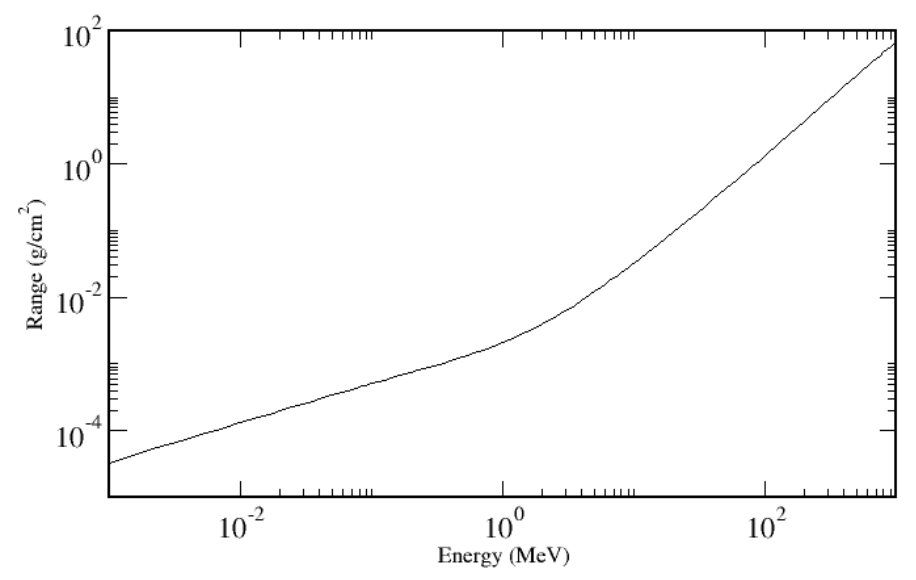

Figure 13. $\alpha$ particles range in CsI(Tl) obtained with the use of the ASTAR software from NIST.

CsI(Tl) We performed the first measurements with the ${ }^{241} \mathrm{Am}$ source on the $\mathrm{CsI}(\mathrm{Tl})$ crystal. The PD signal is shown in figure 14. The low energy tail is due to the $\alpha$ particles that have interacted with the Teflon wrapping. In addition there are also interactions with the air $(2 \mathrm{~mm})$ separating the source from the crystal. We have fitted only the right part of the peak with a Gaussian, as shown in blue in figure 14. From the fit we derive the mean value and the width of the full energy $\alpha$ particle,

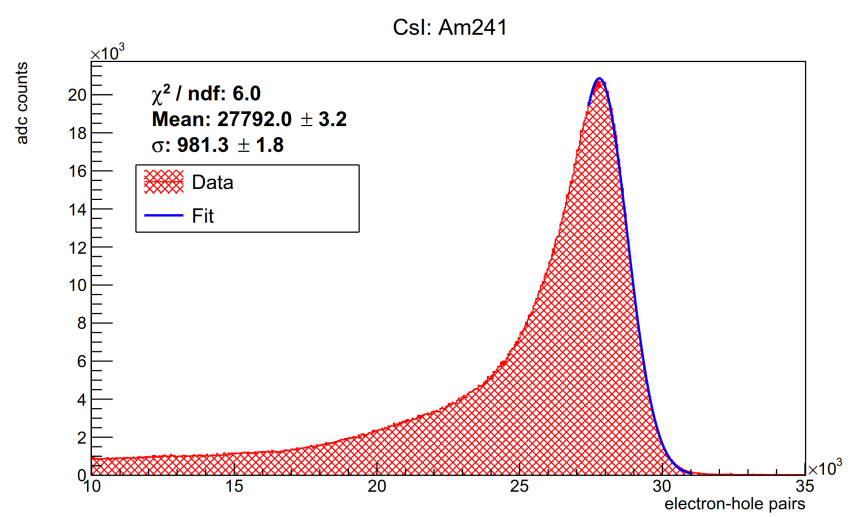

Figure 14. Measured $\alpha$ particles energy in the CsI(Tl) crystal (red) and Gaussian fit of the peak (blue).

with the resolution evaluated as the ratio between the $\sigma$ and the mean value of the Gaussian fit. For $\mathrm{CsI}(\mathrm{Tl})$ the results are: mean value equal to $27792 \pm 3$ electrons and resolution of $3.5 \%$.

BGO We performed the same measurement for the BGO scintillator. The analysis is more complicated because the signal is lower and superimposed with the background pedestal. We thus acquired two sets of data, one of only the background spectrum (i.e. without the source) and the other of the background and the $\alpha$ source signals. We fitted the background only spectrum with a Gaussian to find the pedestal distribution parameters. We then fitted the second spectrum with 
a sum of two Gaussian, in which only the $\alpha$ peak parameters were left free while the background parameters were fixed to the values obtained from the first fit. The results of the second fit are shown

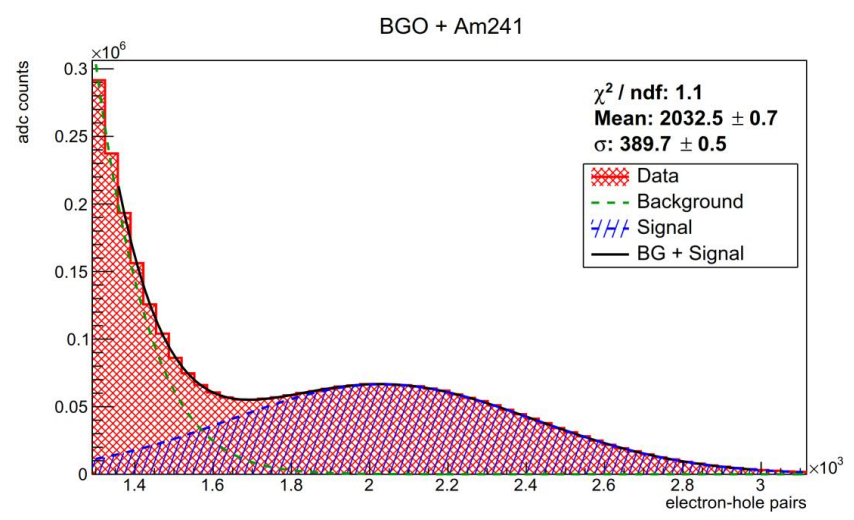

Figure 15. Measured $\alpha$ particles energy in the BGO crystal (red), pedestal Gaussian fit (green), $\alpha$ particle Gaussian fit (blue) and sum of both fits (black).

in figure 15 , in which the measured total spectrum is the red hatched plot. The green, blue and black lines show respectively the background distribution estimated with the first fit, the $\alpha$ particle fit estimated with the second fit and the sum of both fits. For BGO the results are: mean value equal to $2032 \pm 1$ electrons and resolution of $19.2 \%$.

LYSO The LYSO scintillator measurement procedure is the same as the one for the BGO crystal. In addition to the low signal level, LYSO has also a natural radioactivity caused by the lutetium present in the scintillator. This radioactivity generates signals even in absence of an
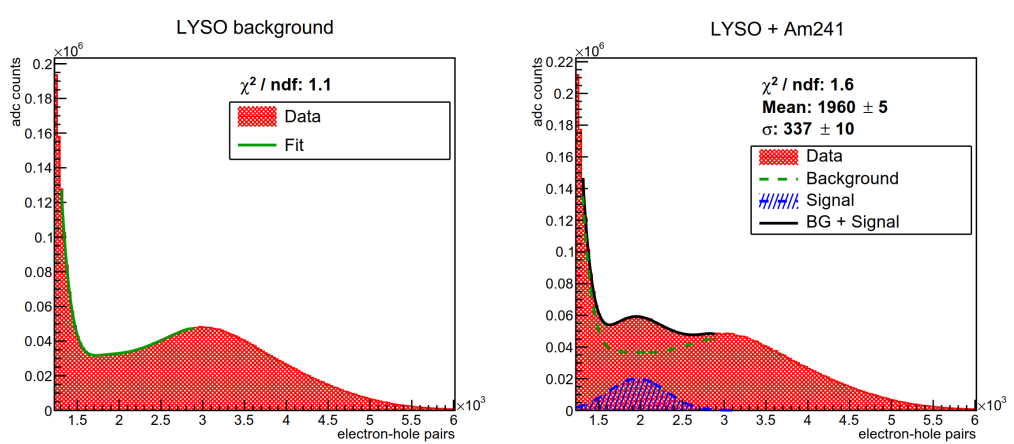

Figure 16. Measured pedestal and natural radioactivity spectrum for the LYSO crystal (red) and in green the region where the source signal is expected to be present (left). Measured $\alpha$ particles energy in the LYSO crystal with the $\alpha$ energy Gaussian fit (blue) and sum of both (black) (right).

external source and has a distribution that can not be fitted by a Gaussian. The spectrum measured in the absence of the ${ }^{241} \mathrm{Am}$ source is shown in figure 16 where in addition to the pedestal, there is a second peak at a higher value due to the natural radioactivity. The spectrum generated by the internal radioactivity of LYSO is asymmetrical and of not obvious parametrisation. We opted to fit the additional part of the spectrum with a polynomial function. The result of such fit with a six degree polynomial plus a Gaussian for the pedestal, is shown at left in figure 16. For the analysis 
of the ${ }^{241} \mathrm{Am}$ spectrum, we then subtracted the green part shown in figure. In practice we fitted an additional Gaussian function in the green region of the spectrum were the ${ }^{241} \mathrm{Am}$ signal manifests itself. The results of the fit are illustrated at right figure 16, in which the signal of the $\alpha$ particles (blue) falls in between the pedestal and natural radioactivity peaks. On this figure, the green and red line represents the background fit, the blue line presents the signal fit and the black one is a sum of all functions. For LYSO the results are: mean value equal to $1960 \pm 5$ electrons and resolution of $17.2 \%$.

The summary of all measurements is reported in table 6 . The measured amplitude summarised in table 6 are not in good agreement with the values presented in table 5. As pointed out in the

Table 6. Amplitude and resolution of the signal generated by $\alpha$ particle $5.5 \mathrm{MeV}$ of ${ }^{241} \mathrm{Am}$ in three scintillators.

\begin{tabular}{|c|c|c|c|}
\hline Scintillator & Mean (electrons) & Mean (\% of CsI(Tl)) & Resolution for a 5.5 MeV $(\alpha)$ \\
\hline CsI(Tl) & $27792 \pm 3$ & 100 & $(3.5 \pm 0.01) \%$ \\
BGO & $2032 \pm 1$ & $(7.31 \pm 0.01) \%$ & $(19.2 \pm 0.1) \%$ \\
LYSO & $1960 \pm 5$ & $(7.05 \pm 0.02) \%$ & $(17.2 \pm 0.5) \%$ \\
\hline
\end{tabular}

presentation of the scintillators characteristics, the literature has different values depending on the sources. An additional reason for the disagreement could be due to the particle source: the light yield of scintillators usually refers to $\gamma$ rays and can be different for other kind of particles. There are few works with direct comparison of these three scintillators and even in those where LYSO is mentioned, it is not possible to deduce the absolute value of the light yield [30].

The light yield measured with $\alpha$ particles can be useful for comparisons, but measurements with MIPs are more pertinent to our calorimetric application. In fact an alpha releases a large amount of energy in a very small volume at the surface of the crystal, in a way that is substantially different from that of a MIP or a particle shower. Cosmic rays provide an easy to use source, but given the crystal dimensions, such measurements are limited by a low rate of the order of $0.01 \mathrm{~Hz}$. For this reason we performed cosmic ray measurements only on full sized final crystals $\left(36 \times 36 \times 36 \mathrm{~mm}^{3}\right)(\mathrm{see}$ section 2.7). Results on the many high energy particle test beams we performed during these years on the various prototypes will be reported in a dedicated paper that is in preparation.

\subsection{Full sized crystal calibration}

The light yield results presented in the previous section, together with the considerations on costs and availability, have led us to choose $\mathrm{CsI}(\mathrm{Tl})$ as the scintillator for the full sized CaloCube crystals $\left(36 \times 36 \times 36 \mathrm{~mm}^{3}\right)$. We procured roughly 700 of these crystals in order to build the prototype to be used on test beams. We performed some preliminary tests on the light yield of a few of these full sized crystals with the same ${ }^{241} \mathrm{Am}$ source used in the previous tests. The crystals were covered with two layers of Teflon for a total thickness of $100 \mu \mathrm{m}$ and the source was placed on the opposite side with respect to the position of the PD. The crystal under test with attached PD is shown in figure 17.

The measured spectrum is shown in the right part of figure 17: the green line shows measured data and the red line is the gaussian fit. The fit has been done for the right part of the peak only, because of the non-Gaussian tail on the left, which is due to the energy losses in the wrapping 


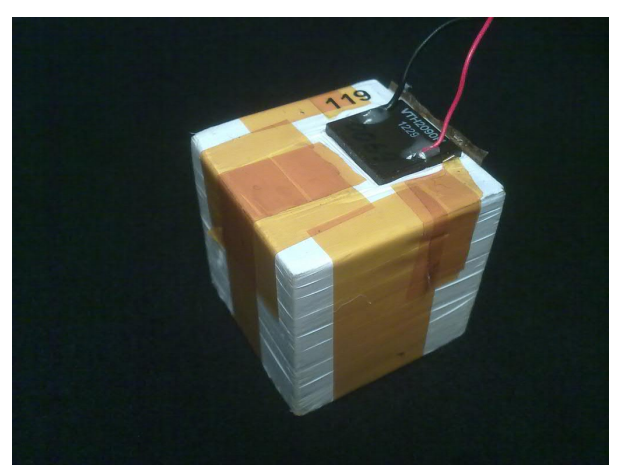

(a)

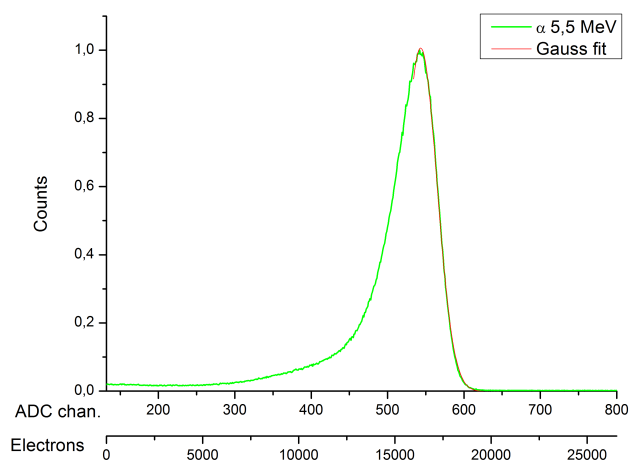

(b)

Figure 17. Full sized CsI(Tl) cubic crystal with the $100 \mu \mathrm{m}$ Teflon wrapping and the VTH2090 photodiode (a). ${ }^{241} \mathrm{Am} \alpha$ particle spectrum measured with a full sized CsI(Tl) crystal (b).

material (cf. section 2.4). Accordingly we estimate a signal of about 16300 photoelectrons with an energy resolution of $5.2 \%$.

We derive the number of expected photoelectrons from the following equation:

$$
S_{e}=L_{p h} \cdot E \cdot L \cdot F_{g} \cdot Q_{P D}
$$

where $\mathrm{S}_{e}$ is the signal level in photoelectrons, $\mathrm{L}_{p h}$ is the light yield of the scintillator, $\mathrm{E}$ is the energy deposited in scintillator (5.5 $\mathrm{MeV} \alpha$ particle), $\mathrm{L}$ is the light collection efficiency in the scintillator, $\mathrm{F}_{g}$ is the geometric factor (i.e. the ratio of the scintillator side area and the photodiode active area), and $\mathrm{Q}_{P D}$ is the quantum efficiency of the photodiode.

We obtained an estimate of the order of 15000 photoelectrons, indicating a qualitative agreement with the measured value. In fact there are many uncertainties in this estimate, not least the light yield of the scintillator and the light collection efficiency, that make it very difficult if not impossible to achieve a reliable quantitative agreement. While the light yield depends on the chosen manufacturer, the light collection efficiency value and reproducibility depend on the crystal surface finish and chosen wrapping material. We thus peerformed an extensive study starting with simulations that were then compared with actual measurements.

\subsection{Light collection efficiency}

We have performed a systematic study of light collection efficiency for various wrapping materials on our full sized crystal. We have performed various simulations, following previous studies [31-34], and focusing our efforts on Teflon, aluminized mylar, and an enhanced specular reflector (Vikuiti [35]). We have also varied the crystal surface finish from matt to fully polished. The actual measurements were made with a full sized CsI(Tl) cube coupled to a VTH2090 PD using an ${ }^{241} \mathrm{Am}$ $\alpha$ source to generate the scintillation signal.

\subsubsection{Simulation}

To acquire a feeling on the issue at hand, we performed optical ray tracing simulations using GEANT. The initial photons were produced in the crystal following either a MIP interaction from 
any arbitray direction or an electromagnetic shower from $100 \mathrm{GeV}$ electrons incident on a nonscintillating absorber situated in front of the crystal. The following parameters were varied in the simulations: surface finish (roughness $\sigma_{\alpha}$ in the range $0.0 \div 0.5$ radiant) and wrapping material (no wrapping, Teflon, aluminized mylar). The light detection efficiency $\epsilon$ seen in the plots of figure 18 is defined as the ratio of the number of photons detected by the PD to the number of photons produced in the scintillation process (thus it includes also the quantum efficiency of the PD). In

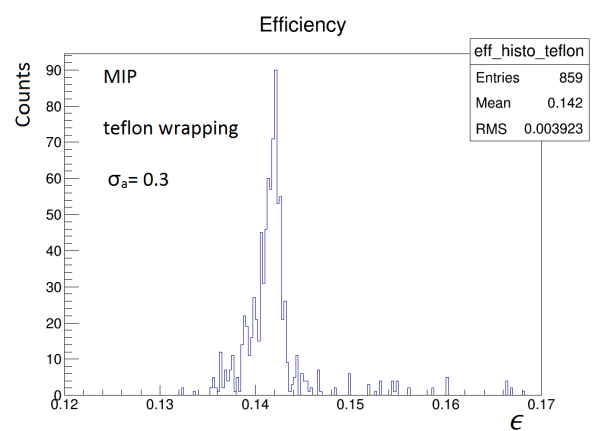

(a)

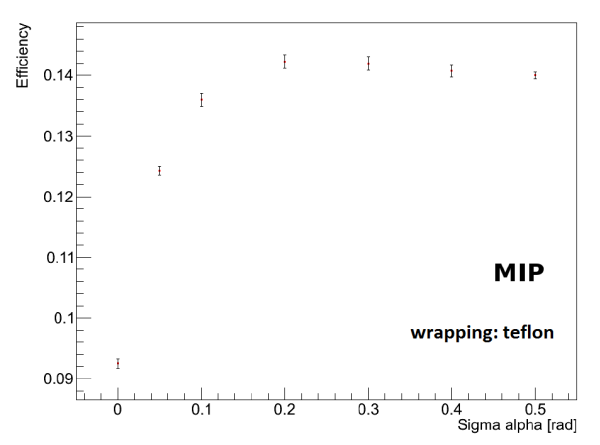

(c)

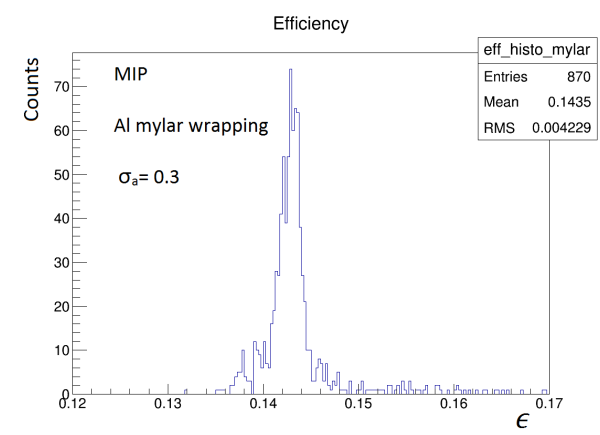

(b)

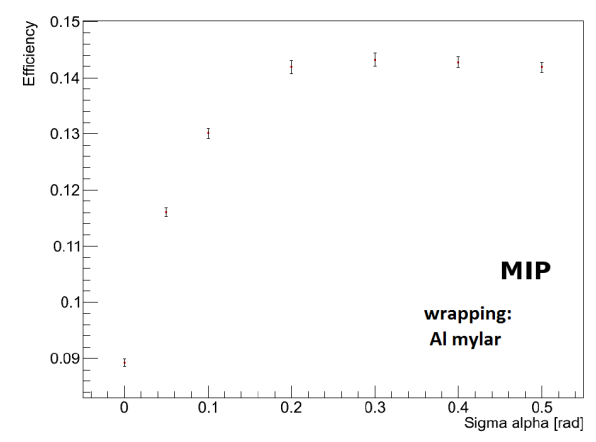

(d)

Figure 18. Efficiency distributions generated by MIPs in the crystal with Teflon and Aluminised Mylar wrapping and with roughness $\sigma_{\alpha}=0.3$ (Top). Efficiency as a function of roughness $\left(\sigma_{\alpha}\right)$ for Teflon and Aluminised Mylar (Bottom).

figure 18 a few examples of the results obtained are shown. The top two plots in the figure, are typical efficiency distributions obtained by considering the photons collected in the PD area from all simulated particle interactions. These distributions were then fitted with Gaussians to obtain the respective central values. The values obtained are shown in the bottom part of figure 18 as a function of roughness $\sigma_{\alpha}$ for two choices of wrapping material.

For Teflon or aluminized mylar wrapping, the light collection efficiency starts from $9 \%$ for a polished surface and grows rapidly with $\sigma_{\alpha}$ up to $14 \%$. This behaviour is consistent with the fact that a rougher surface diffuses the photons and thus reduces the amount of reflections needed to arrive at the PD entrance window [36]. This effectively reduces the probability that the photon will be absorbed by the wrapping (because of the lower number of reflections) or in the volume of the crystal (because of the shorter path travelled).

We have found, that in the case of a crystal without wrapping, the efficiency descends from roughly 
$6 \%$ for a polished crystal to a stable value of less than $2 \%$ with increasing surface roughness. This is expected, since an internal photon will be more likely to undergo total reflection if the crystal surfaces are polished than if they are sanded (causing diffusion to the outside to occur).

The results of the many simulations we performed, are in agreement with previous data [36] available for the crystals with comparable dimensions. Without wrapping, crystals with polished surfaces (low $\sigma_{\alpha}$ ) show the highest efficiencies, whereas crystals with rough (diffusive) surfaces show better performance when using both reflective and diffusive wrappings.

\subsubsection{Measurements}

Using our simulations as a guiding input, we chose to have a matted crystal surface finish. Thus all the full sized crystals used for the Calocube project were sanded on five sides and polished only on the side where the PDs are glued. On some of the CsI(Tl) crystals we have performed various light collection efficiency measurements with different wrapping materials (Teflon, aluminised mylar, Vikuiti [35]). These were then repeated for various Teflon thicknesses. We also measured the uniformity of response as a function of the ${ }^{241} \operatorname{Am} \alpha$ source position.

\section{Aluminised Mylar foil and Teflon}

For this measurement we reverted to small sized crystals used in the scintillator comparison. The CsI(Tl) cubes were wrapped with aluminised Mylar and with Teflon wrapping. Each crystal was coupled to VTH2090 PDs and tested with an ${ }^{241} \mathrm{Am} \alpha$ source. An aperture of $2 \mathrm{~mm}^{2}$ was opened in the wrapping to let the $\alpha$ particles penetrate inside the crystal. Typical spectra measured and

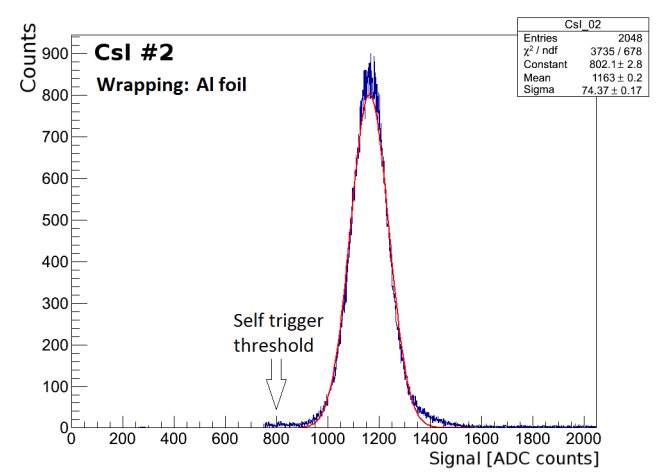

(a)

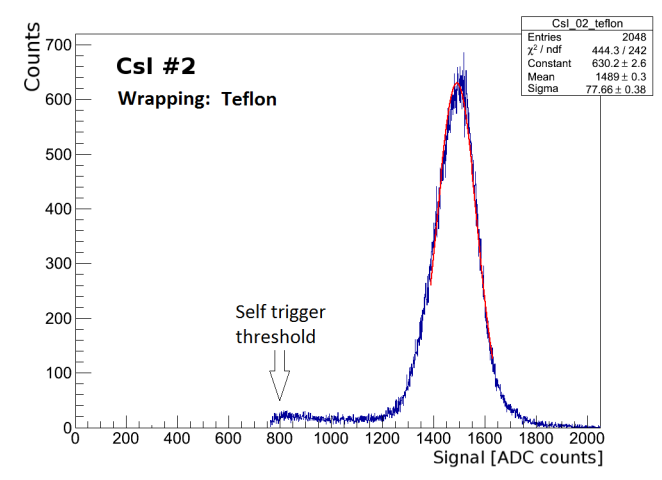

(b)

Figure 19. ${ }^{241} \mathrm{Am} \alpha$ particle spectrum from $\mathrm{CsI}(\mathrm{Tl})$ crystal with aluminum foil (a) and $50 \mu \mathrm{m}$ Teflon (b) wrapping (blue line) and Gaussian fit of the peak (red line). A relatively high threshold was used in these plots to eliminate random self triggered events due to external pickup. The pedestal value of 203 ADC channels was taken from figure 10 .

their Gaussian fits, are shown in figure 19(a) and 19(b) for aluminised Mylar and Teflon wrapping respectively. We found that a Teflon wrapping gives a signal increase of about of $35 \%$, while the energy resolution is improved up to roughly $\sim 6 \%$ for both crystals. The results for two crystals with both wrappings are presented in table 7 . Both show a marked improvement from aluminised Mylar to Teflon. 
Table 7. Gaussian fit results (amplitude and resolution) for two similar crystals, using the $5.5 \mathrm{MeV}$ emission peak of of ${ }^{241} \mathrm{Am} \alpha$ particle. Results are consistent within $10 \%$.

\begin{tabular}{|c|c|c|c|c|}
\hline CsI(Tl)+PD & Wrapping & Centroid [ADC ch.] & $\sigma$ [ADC ch.] & Resolution \% \\
\hline 2 & Al & 960 & 74 & 7.7 \\
& Teflon & 1286 & 78 & 6.1 \\
3 & Al & 1068 & 90 & 8.4 \\
& Teflon & 1435 & 90 & 6.2 \\
\hline
\end{tabular}

\section{Light collection efficiency for different Teflon thicknesses}

We used full sized $\mathrm{CsI}(\mathrm{Tl})$ cubic crystals to study the influence of Teflon wrapping thickness. We started with two layers of Teflon tape $(\sim 100 \mu \mathrm{m})$ which was a standard configuration used on a small (roughly 100 crystals) Calocube protoype used to collect preliminary electromagnetic shower data. The $\alpha$ spectrum was measured every time after adding two additional layers of Teflon, till

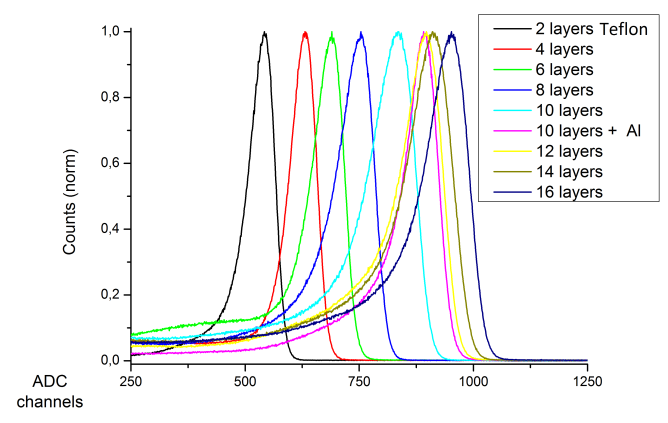

(a)

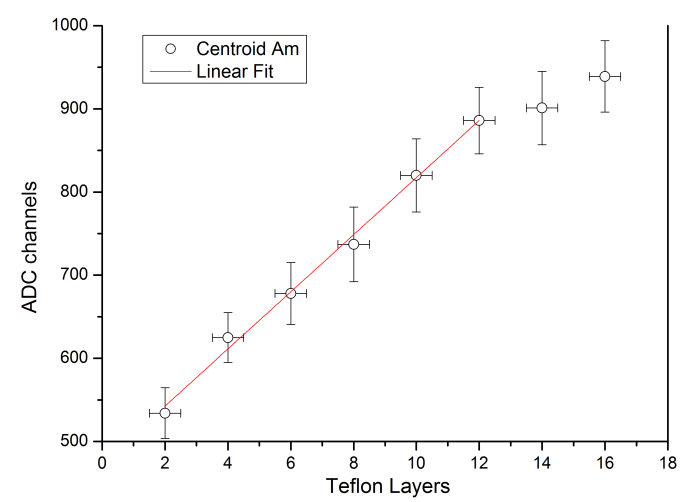

(b)

Figure 20. ${ }^{241} \mathrm{Am} \alpha$ particle spectra measured by $\mathrm{PD}+\mathrm{CsI}(\mathrm{Tl})$ for different thicknesses of Teflon wrapping (a). Signal amplitude (centroid of the previous spectra) dependence on Teflon thickness (b).

we reached a 16 layers $(800 \mu \mathrm{m})$ thickness. After 10 layers we also added an aluminised Mylar external wrapping which further increased the signal measured. All the spectra are shown in figure 20. Each peak has been fitted with a Gaussian function and plotted as a function of Teflon thickness. The peak and width values are listed in table 8 .

Table 8. Gaussian fits (amplitude and width in ADC channels) for different thicknesses of Teflon wrapping. Peak values are different from what previously shown due to different crystal size and different scintillator manufacturer.

\begin{tabular}{|c|c|c||c|c|c|}
\hline Teflon layers & Peak $($ ADC ch. $)$ & $\sigma($ ADC ch. $)$ & Teflon layers & Peak & Width \\
\hline 2 & 534 & 30.6 & 10 & 820 & 43.9 \\
4 & 625 & 30.1 & 12 & 886 & 40 \\
6 & 678 & 37.3 & 14 & 901 & 44.1 \\
8 & 737 & 44.8 & 16 & 939 & 43 \\
\hline
\end{tabular}


We have found that the signal with 16 Teflon layers wrapping increases by a factor $\sim 1.76$ respect to the signal with only 2 Teflon layers. The signal amplitude rises linearly with the number of Teflon layers up to 12 layers $(600 \mu \mathrm{m})$. After 12 layers, the signal increase becomes less pronounced and begins to flatten out even 14-16 layers. The measurements indicate that thicker layers of Teflon wrapping result in better performance. Also the resolution increases reaching values of the order of 5\%. In practice though, this is difficult to implement as the wrapping with so many layers (plus the aluminised Mylar) is a time consuming procedure that also increases dead space in the actual calorimeter.

\section{Comparison between Teflon and Vikuiti}

Vikuiti Enhanced Specular Reflector (ESR) is an ultra-high reflectivity, mirror-like optical enhancement film that is widely used in the industry [35]. The thin, non-metallic, $100 \%$ polymer film, built with multi-layer polymer technology, has a $98 \%$ reflectance across the visible spectrum. Vikuiti ESR is an excellent specular surface reflector. We decided to test this type of wrapping on one of the full sized crystals to see how it performed respect to the Teflon wrapping. The signal distribution measured with the crystal wrapped with two Teflon layers and then with Vikuiti is shown in figure 21(a) and 21(b) respectively. The Gaussian fits to the spectra give a peak value of 230 ADC channels for the 2 layer Teflon wrapping and 418 ADC channels for Vikuiti. While the absolute ADC values are different from the other measurements presented in this section (534 8) because of a different gain setting on our AMPTEK amplifier, the increase by a factor of 1.8 is the same as that obtained previously with a 16 layer Teflon wrapping. This result was confirmed with more measurements on

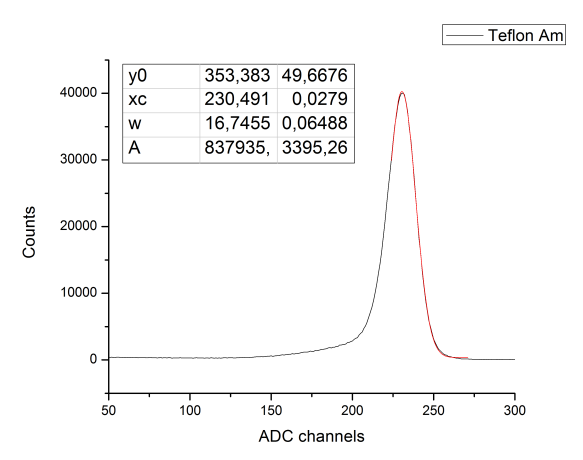

(a)

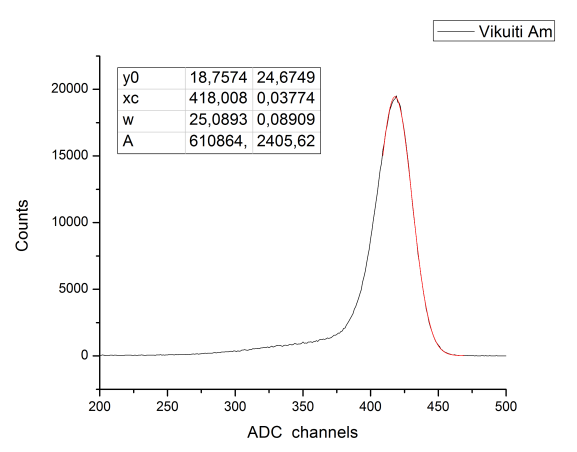

(b)

Figure 21. $\quad{ }^{241} \mathrm{Am} \alpha$ particle spectrum from CsI(Tl) crystal with $\sim 100 \mu \mathrm{m}$ Teflon (a) and Vikuiti (b) wrapping (black line) and Gaussian fit of the peak (red line).

more full sized crystals, we thus chose to use Vikuiti wrapping for all the CsI(Tl) crystals (roughly 700) used for the Calocube protoype.

\section{Light collection uniformity for the different crystal faces}

We also performed various measurements regarding the uniformity of light collection as a function of the $\alpha$ source placement, in particular the crystal face used. We measured the signal amplitude, with the source placed in front of each different face of the crystal, using a full sized CsI(Tl) crystal with a 100 micron Teflon wrapping. A schematic view of the PD-crystal setup and the six positions of the ${ }^{241} \mathrm{Am}$ source used for the measurements is shown in figure 22(a). For each data acquisition the source was placed in the centre of the corresponding face (with an aperture of 2 


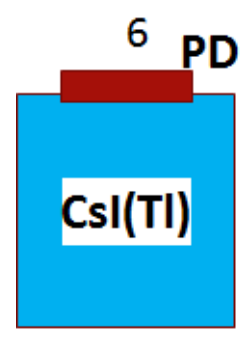

1

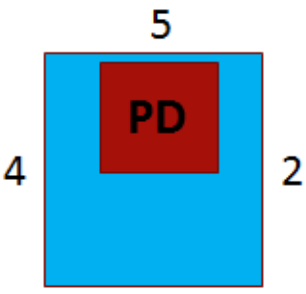

3

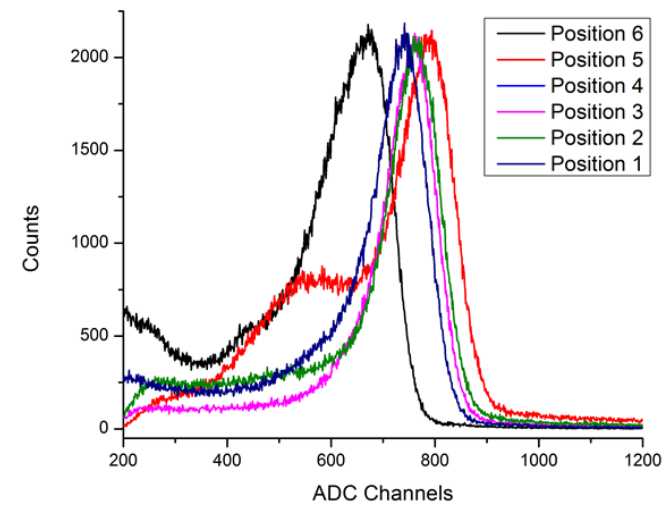

(b)

Figure 22. Schematic view of PD and source positions with respect to the scintillating cubic crystal (a). Signal amplitude dependence on source position. Position 5 has a significant shoulder which is probably due to a slight misalignement of the source respect to the aperture in the wrapping material. The ADC scale changes from the one of table 8 due to a different F.E. gain (b).

$\mathrm{mm}^{2}$ ). The acquired spectra are shown in figure 22(b). Position 5, which is the lateral face closest to the PD, gives the highest signal (810 ADC channels). The other faces anyway show similar peak values (order of 780 ADC channels) except for position 6 which shows the lowest signal (650 ADC channels). In fact this is the same face where the PD is glued and photons from the alpha particle have to travel much further in order to reach the PD. These results demonstrate a very good uniformity of response especially when considering that they were obtained with a two layer Teflon wrapping. With the Vikuiti wrapping chosen for the prototype the uniformity improves further.

\subsection{CsI(Tl) crystal response with cosmic rays}

All the measurements described so far have used a ${ }^{241} \mathrm{Am} \alpha$ particle source. This has obvious advantages in terms of particle rate and ease of measurements in general but needs to be compared to the crystal response to MIPs since the crystals will be used to build a calorimeter for highly energetic particle showers. We have thus performed some comparisons using a cosmic ray bench whose schematic diagram is shown in figure 23. An external trigger system consisting of two fast plastic scintillators $30 \times 30 \mathrm{~mm}^{2}$, coupled to PMTs and placed above and below the CsI(Tl) scintillator cube selects the cosmic ray events. This trigger is used to drive the Amptek data acquisition. The same system is used in self triggering mode with the $\alpha$ source.

A MIP in the cube with $36 \mathrm{~mm}$ side releases $\sim 20 \mathrm{MeV}$ of energy ${ }^{1}$. This is roughly a factor 4 higher than the energy of our $\alpha$ particle source. From the spectra shown in figure 24 the ratio is a factor two higher than what expected. The Landau fit to the cosmic ray spectrum gives a apeak value of 260000 electrons, while with the same electronic chain and Amptek gain settings the $\alpha$ spectrum peaks at 31000 electrons.

${ }^{1} \mathrm{CsI}(\mathrm{Tl})$ provides the following energy loss for a unit of distance: $1 \mathrm{MIP} / \mathrm{cm}=1.25 \mathrm{MeV} /\left(\mathrm{g} / \mathrm{cm}^{2}\right) \cdot 4.5 \mathrm{~g} / \mathrm{cm}^{3}=5.62$ $\mathrm{MeV} / \mathrm{cm}$. For a CsI cubic crystal of $36 \mathrm{~mm}$ side, $1 \mathrm{MIP}$ has energy loss equals to $5.62 \mathrm{MeV} / \mathrm{cm} \cdot 3.6 \mathrm{~cm} \approx 20 \mathrm{MeV}$ (for orthogonal tracks). 


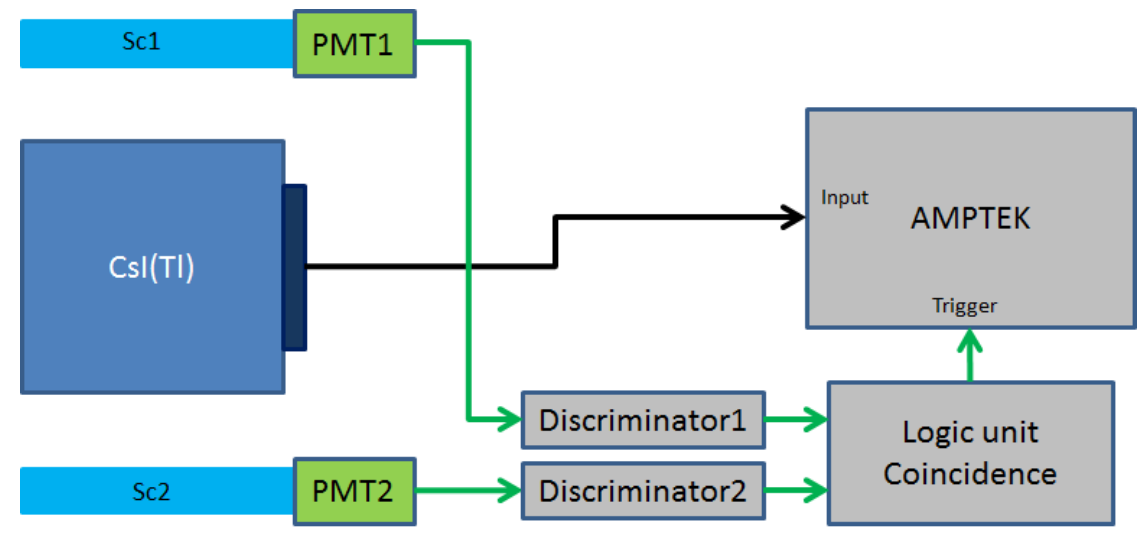

Figure 23. Block diagram of the Cosmic rays measurement bench.

Regarding the disagreement between signals for MIP and $\alpha$ particles, one should consider that a MIP produces scintillation within the whole crystal volume, whereas an $\alpha$ particle only in the small local area near the interaction point at the surface. For a MIP this difference can increase the number of the photons detected per unity of deposited energy because of a higher average light collection efficiency. Other factors that could contribute are: at low energies the light response of a

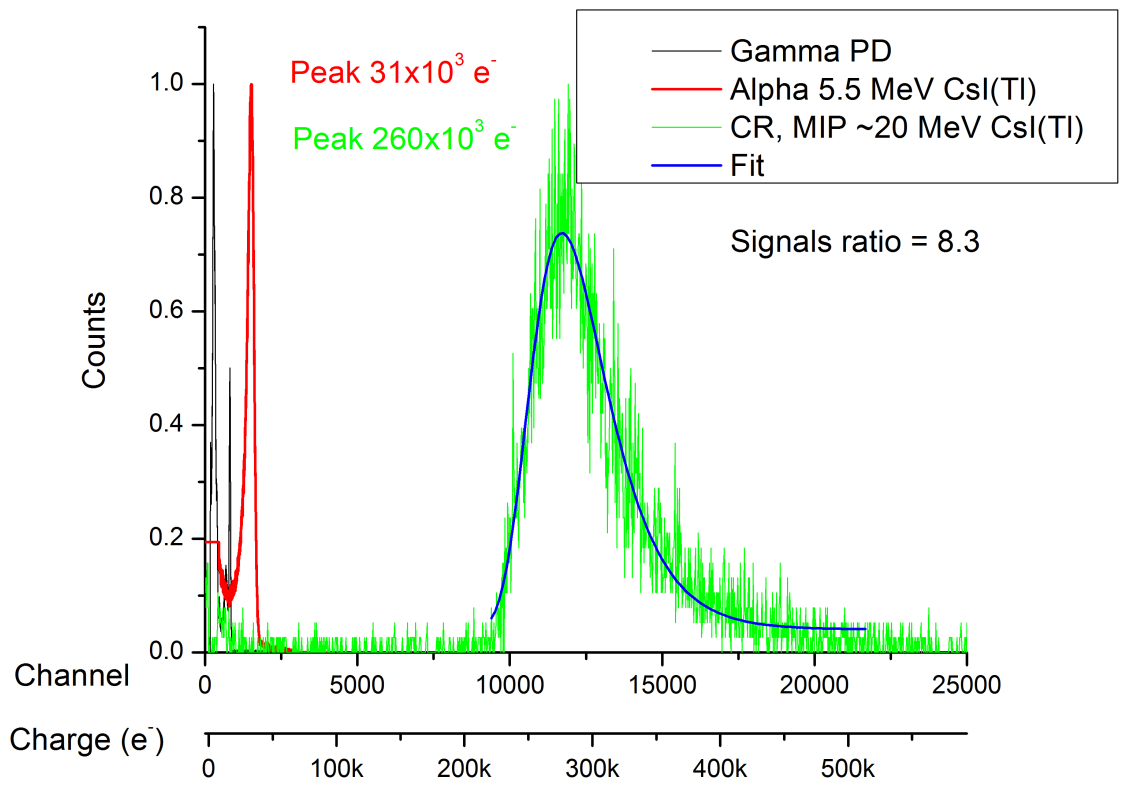

Figure 24. Cosmic rays spectrum (green), compard to the $5.5 \mathrm{MeV} \alpha$ particle (red) response. See text for an explanation of the differences observed. Also plotted is the PD response to the ${ }^{237} \mathrm{~Np}$ lines used for calibration of the electronic chain (see Subsec. 2.3.2).

$\mathrm{CsI}(\mathrm{Tl})$ is known to show a non-linear correlation with deposited energy especially for heavy ions, and a dependence of such correlation on both the charge $\mathrm{Z}$ and mass $\mathrm{A}$ of the detected particle $[33,37]$. These effects also depend on the details of the Tallium doping of the crystal. We have 
used this last measurement as the reference value for the typical signal level and light collection efficiency to be expected for all the Calocube crystals.

\section{Calorimeter mechanical design studies}

We have investigated the mechanical properties of a carbon fibre tray structure large enough to hold a 28 x 28 crystal array. This was an initial foray for a space qualified design that could be brought into orbit by a rocket. We also designed and realised a smaller tray structure using common polyoxymethilene that was used to build a prototype for particle test beam measurements.

\subsection{Carbon fibre tray prototypes and development}

The final Calocube mechanics must use a lightweight support material, structurally compatible with integration in a standard payload for a space mission, so the decision was taken to use trays to support the crystals, made out of space qualified, high modulus carbon fibre (CF) and epoxy resin. The design of these fibre trays was made in collaboration with the LOSON Company[38]
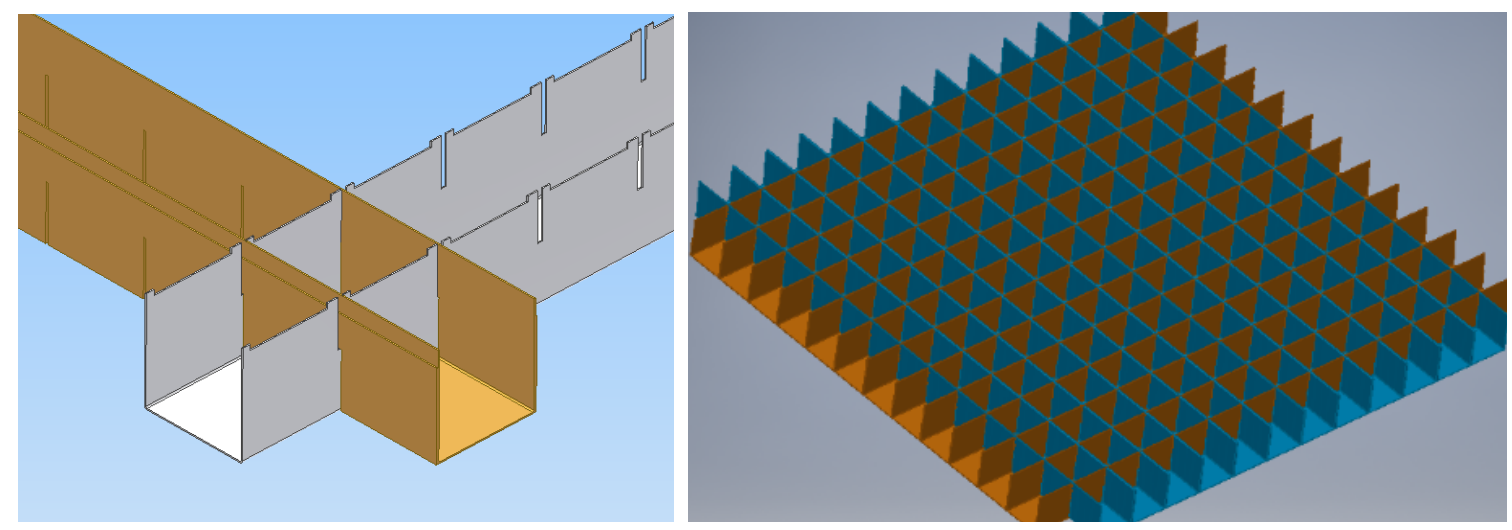

Figure 25. Two U carbon fibre profiles intersecting each other (left), a tray consisting of many intercrossing U profiles (right).

(an Italian company that produces CF components for aerospace and that produced for us the first $6 \times 6$ prototype and the last $28 \times 28$ CF trays). A suitable design was developed that can be used in a production run using a glueing process between basic carbon fibre U-profiles (see figure 25). Each $\mathrm{U}$ profile is obtained by laminating the carbon fibre using custom designed metal moulds. In case the trays require a high number of cells one can group together in a single mould many $U$ profiles reducing the number of steps in the work flow. After lamination but before glueing, the profiles must be reworked (cut-outs, grooves, etc.) using specific custom made tools that not only support the piece, but prevent the eventual de-lamination of the carbon fibre.

In the final calorimeter assembly all these trays are stacked on top of each other and above a support base plate. Four lateral columns run along the four edges to further rigidify the structure (see figure 26). Finally, each tray is shifted, in the $\mathrm{X}$ and $\mathrm{Y}$ directions, by half a crystal width respect to each other, to eliminate unintended "escape corridors" for the impinging particles.

We realised a scaled down 6x6 carbon fibre tray, following the above described construction procedure and drawings (see figure 27), with each cell measuring $38 \times 39 \mathrm{~mm}^{2}$ (x and y), and with 


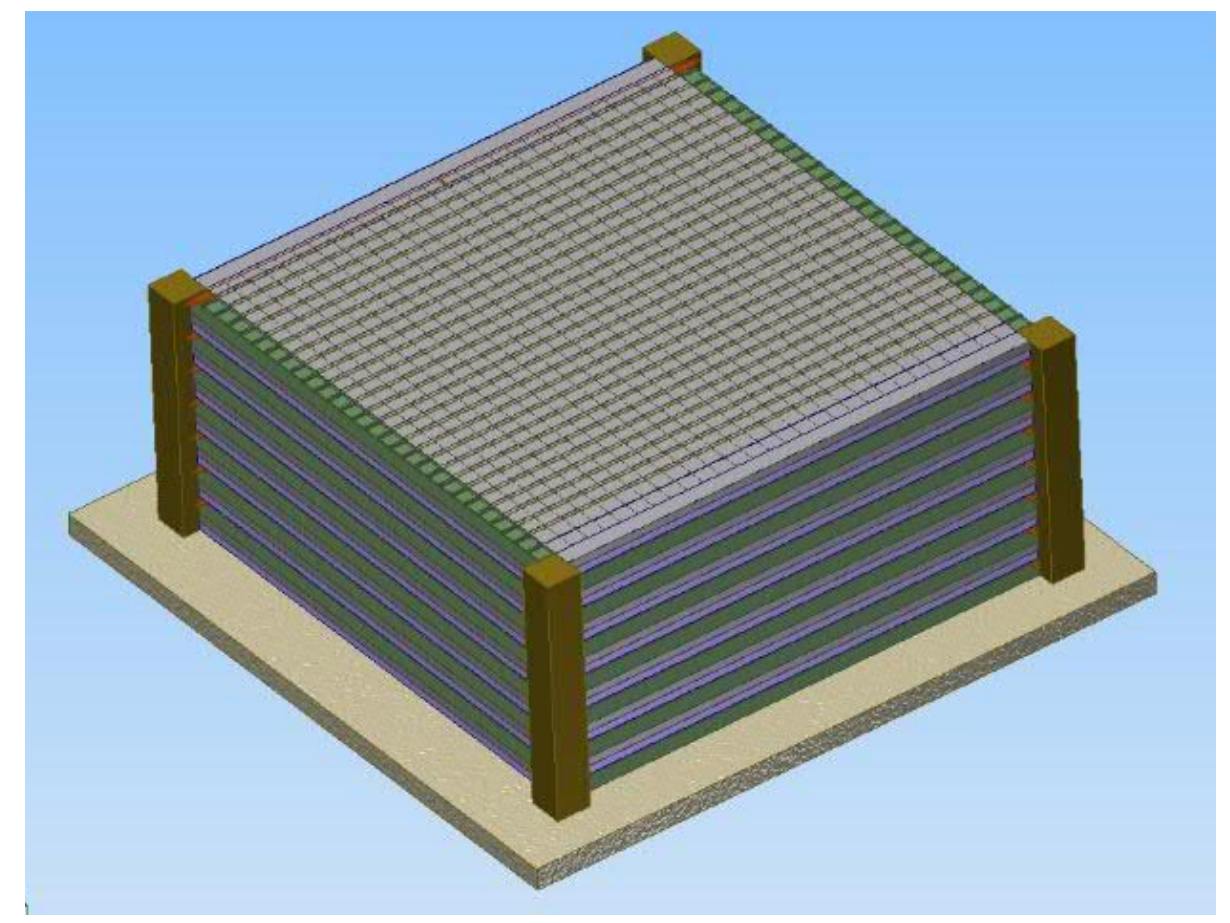

Figure 26. A rendering showing how the carbon fibre trays would be stacked on top of each other in a calorimeter assembly.

a depth of $38.5 \mathrm{~mm} \mathrm{(z).} \mathrm{From} \mathrm{these} \mathrm{drawings,} \mathrm{we} \mathrm{also} \mathrm{derived} \mathrm{a} \mathrm{detailed} \mathrm{simulation} \mathrm{model} \mathrm{used} \mathrm{for}$ verification (see following sections).
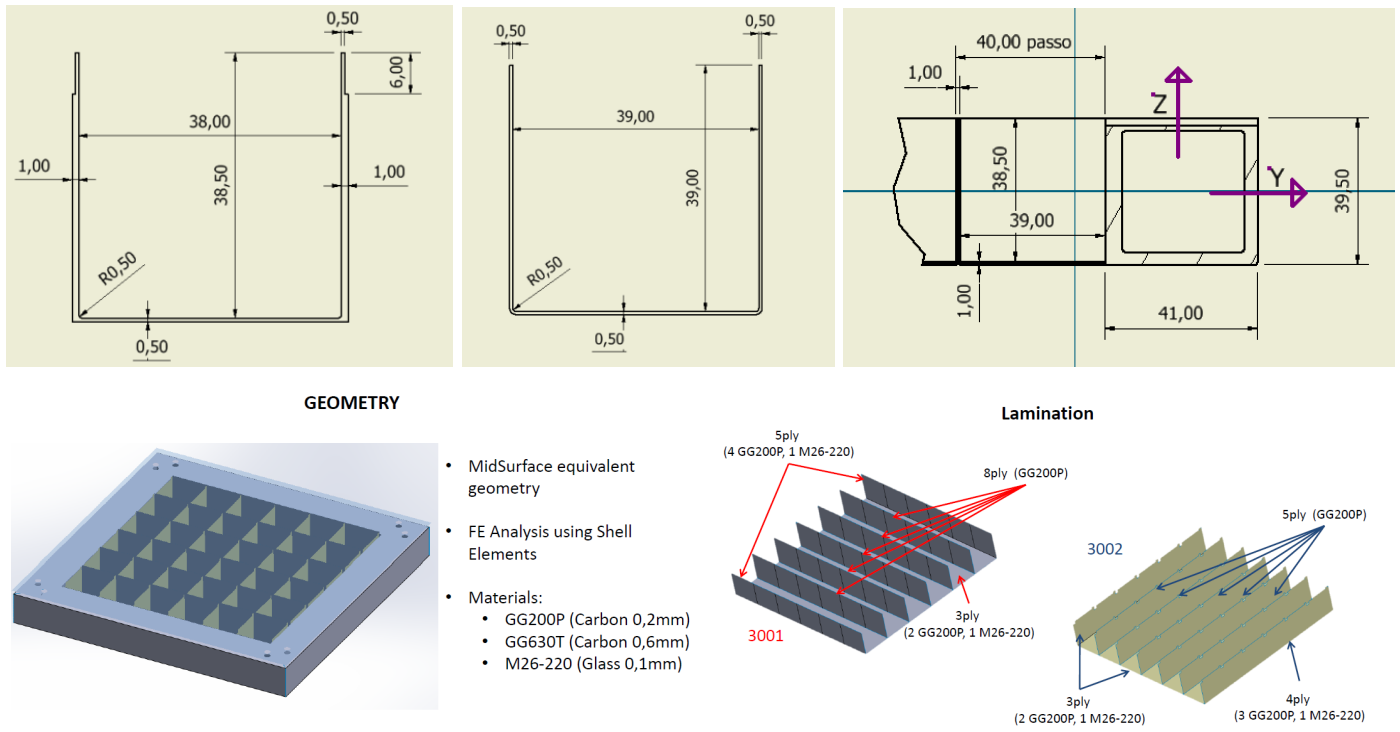

Figure 27. The drawings (TOP), with the relevant dimensions (mm) of the two $\mathrm{U}$ profiles and of the stiff border rim used for the $6 \times 6$ carbon fibre cell prototype. Detailed simulation drawings (BOTTOM) used for verification. 
The border was stiffened with a $40 \times 40 \mathrm{~mm}^{2}$ empty square section (see figure 28 ) of $3 \mathrm{~mm}$ thickness. The prototype built by LOSON was precisely measured at the INFN laboratories in Pisa and the results compared to the original drawing parameters.

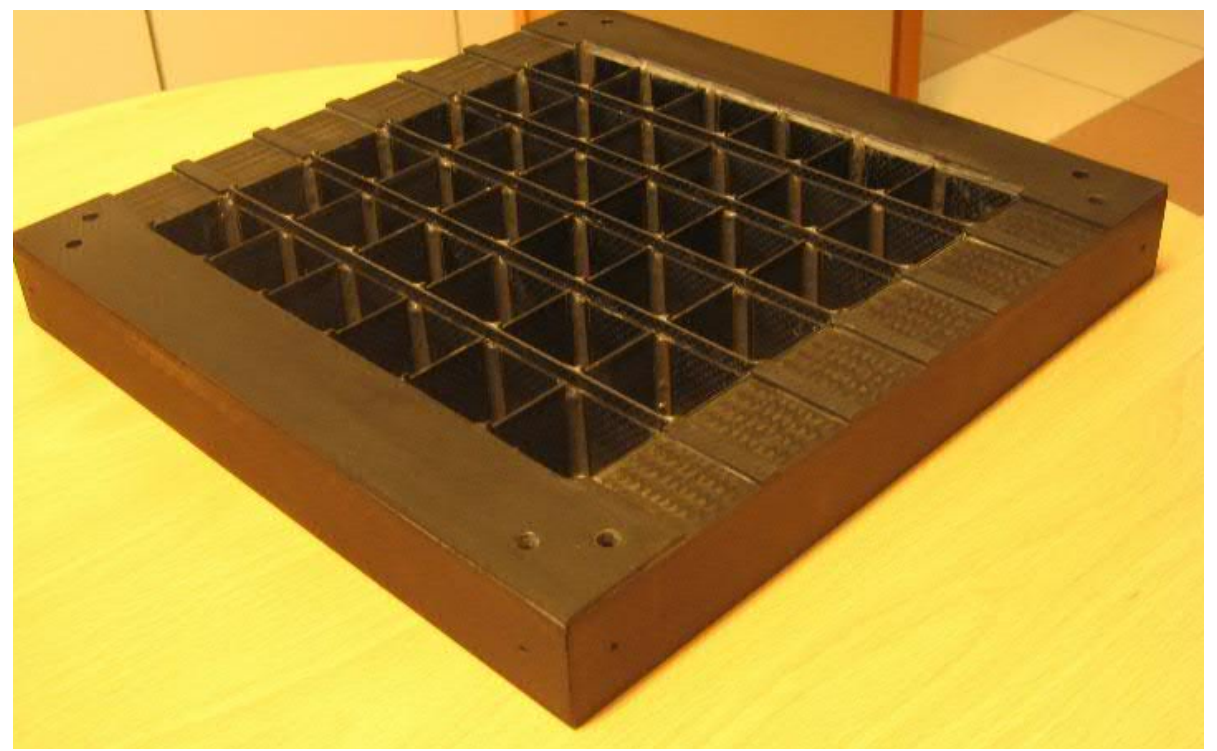

Figure 28. Photo of the $6 \times 6$ carbon fibre prototype used for the verification tests.

\subsection{Carbon fibre tray measurements results}

The measurement results on cell size uniformity and depth of the 6x6 tray are shown in the following plots. From these measurements, obtained with a precision coordinate-measuring machine (CMM) in our clean rooms (see figure 29), it appears that the depth of the cells is less than the design value $(37.5 \mathrm{~mm} \pm 0.5 \mathrm{~mm}$ instead of $38.5 \mathrm{~mm}$ ) which is probably due to the glueing procedure followed during assembly. Also a border effect in the X-Y plane is manifest both in the cell sizes and their pitch (not shown). This prototype was then used for vibration tests in order to find the
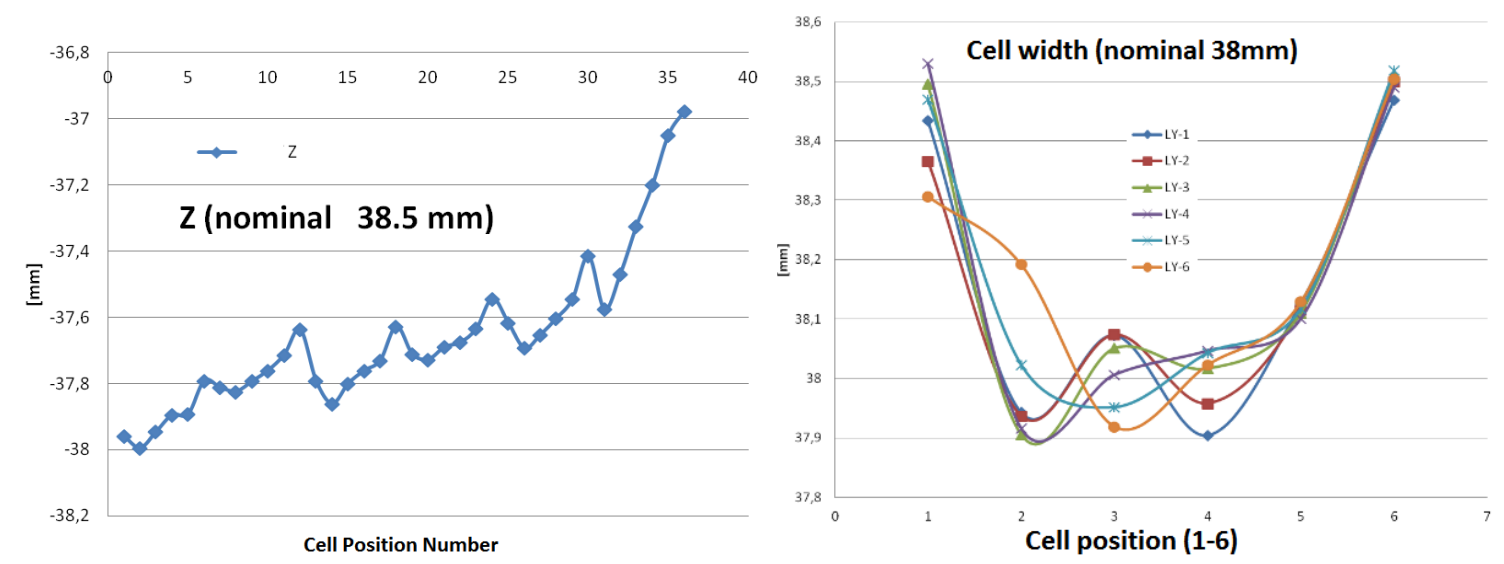

Figure 29. Uniformity measurements: Plots showing the 36 cells depths (left), and their widths (6x6 matrix, cell position along the row for six different columns). 
resonant frequencies, in particular those concerning the $\mathrm{z}$ axis orthogonal to the tray. These tests were performed at the SERMS [39] facility. A drawings and photo of the installation are shown below (see figure 30). The tests performed were first used to check the validity of our Finite Element
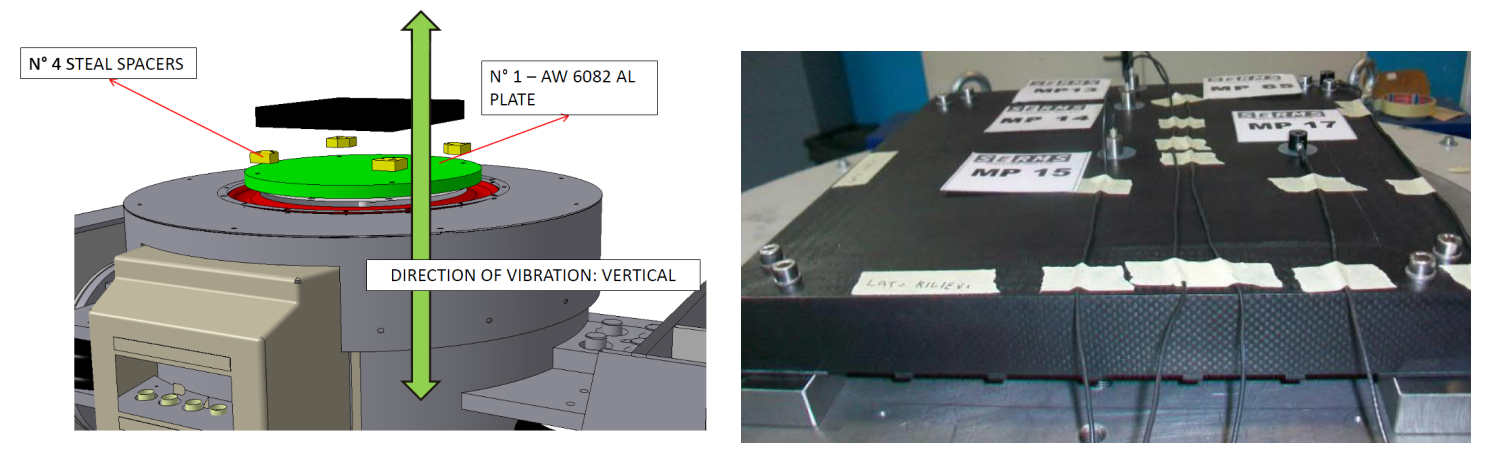

Figure 30. Drawing of the vibrating fixture (left), and photo of the fixation plate and carbon fibre tray with the accelerometers.

Modelling (FEM). The frequency graph (see figure 31) shows a fundamental resonance at $1018 \mathrm{~Hz}$ evident also from the phase change. This frequency was measured with more or less the same intensity on all accelerometers. The test was repeated many times, checking the reproducibility of the results. Sometimes we also exchanged the accelerometers. All measurements gave consistent results. These results are to be compared with the first simulations performed on the $6 \times 6$ cell

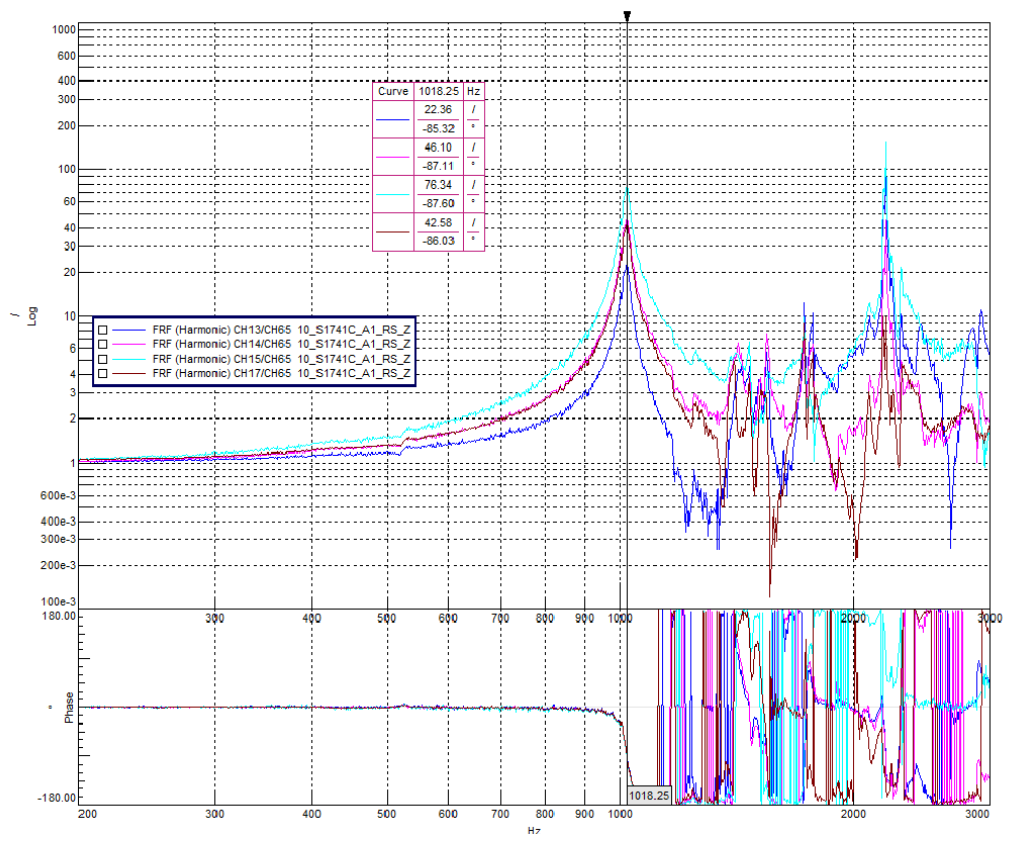

Figure 31. Graph of the resonant frequencies obtained from the $6 \times 6$ cell CF prototype.

protoype. Using the drawings shown in figure 27, the structure was simulated implementing the detailed knowledge of the carbon fibre types and lamination processes used. The tray was simulated with and without a cover lid, which anyway has little effect on the resonant frequency being very 

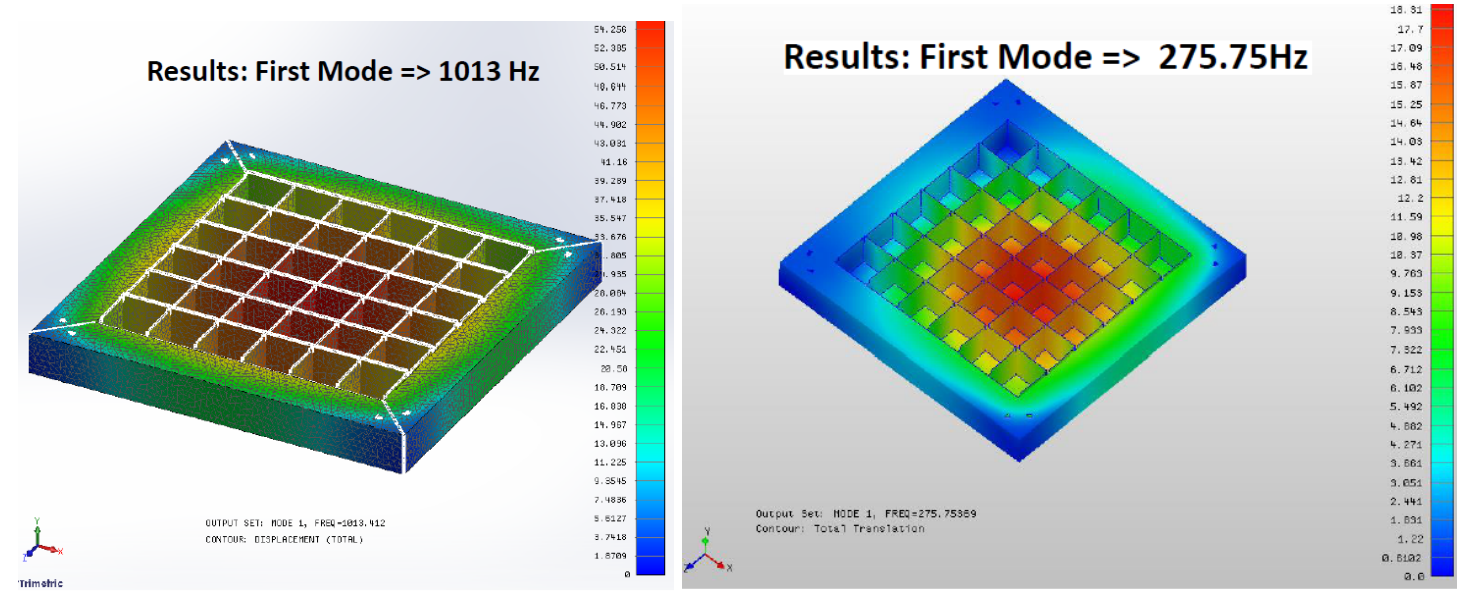

Figure 32. First mode resonant frequency of the simulated tray: with no crystals (left), and loaded with crystals (right). The weight of the 36 crystals is roughly $8 \mathrm{Kg}$. Both configurations are without cover lid.

thin $(\sim 1 \mathrm{~mm})$. The results indicate, as expected, a strong vertical displacement at the centre of the tray with the resonant frequency increasing from $882 \mathrm{~Hz}$ to $1013 \mathrm{~Hz}$ depending on the boundary conditions implemented (see figure 32 ) in the simulations. The results match very well what was found in the tests at SERMS in Terni (figure 30) where the tray borders were fixed to the supporting plate. In simulations, the tray was also loaded with the crystals which provide an increase in mass of the structure with no additional structural support or added rigidity. As shown in the right picture of figure 32 the resonant frequency, in this case, drops dramatically to less than $300 \mathrm{~Hz}$ with a significant doubling of the vertical displacement at the centre. Given the typical power-spectraldensity vibration levels of a rocket, such a displacement would translate into certain destruction of the tray as it is way beyond the deformation that the CF structure can tolerate. These results
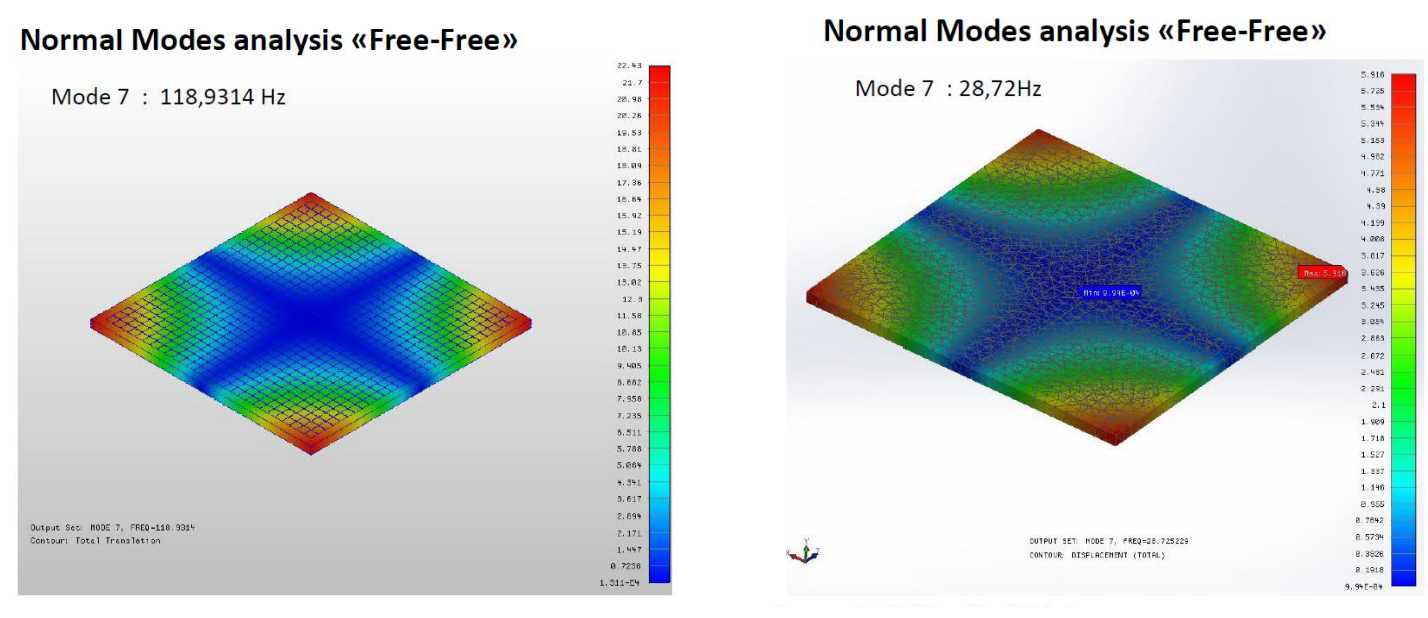

Figure 33. First mode resonant frequency of a simulated full sized $28 x 28$ cell tray: with no crystals (left), and with crystals (right). The weight of the crystals in the simulation is $165 \mathrm{Kg}$.

were expanded to the simulation of a full scale $28 \times 28$ cell CF tray, where the first mode resonant frequency dropped to around $100 \mathrm{~Hz}$, (figure 33, left). Once loaded with crystals the frequency 
dropped further to 29 Hertz (figure 33, right). Vertical displacements were reduced by lowering the input stimulus. Such a low frequency poses a serious risk during the launch phase of the experiment. The trays suffer considerable deformation and will break and impact one on top of the other. The finite element simulations performed by LOSON have confirmed that the stiffness of the $28 \times 28$ tray is too low to cope with the load of the crystals, totaling about $165 \mathrm{~kg}$.

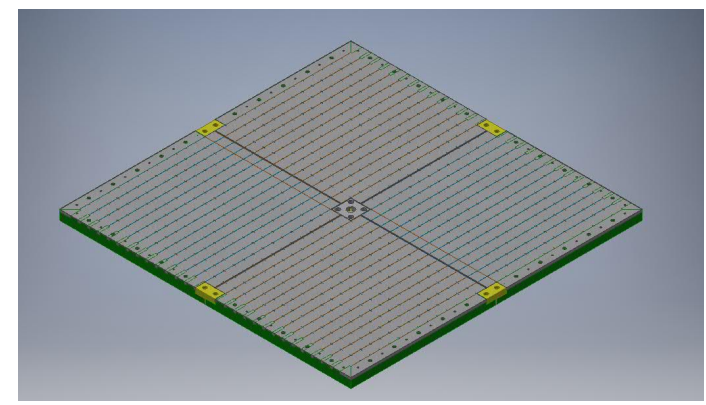

(a)

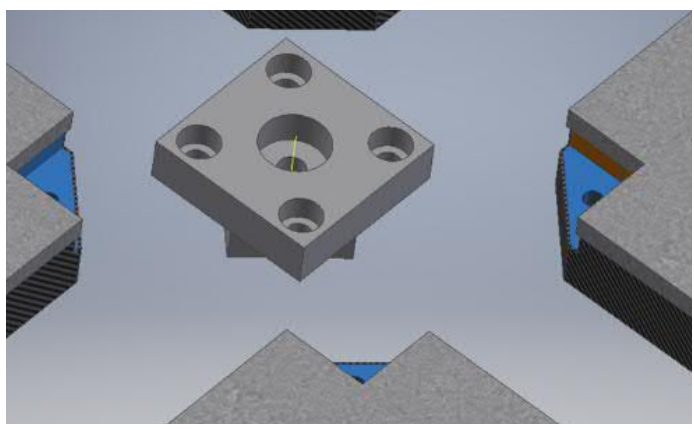

(c)

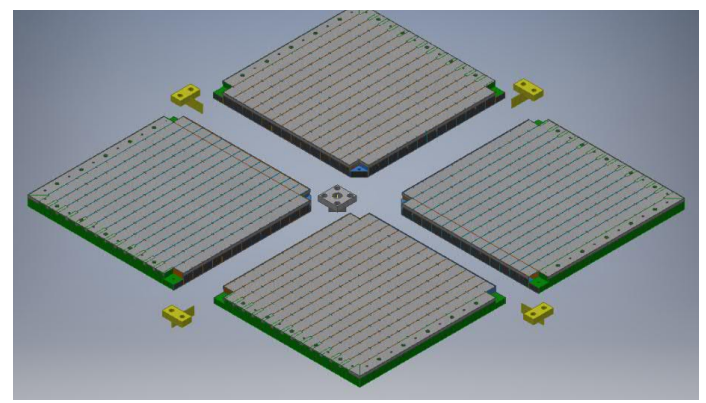

(b)

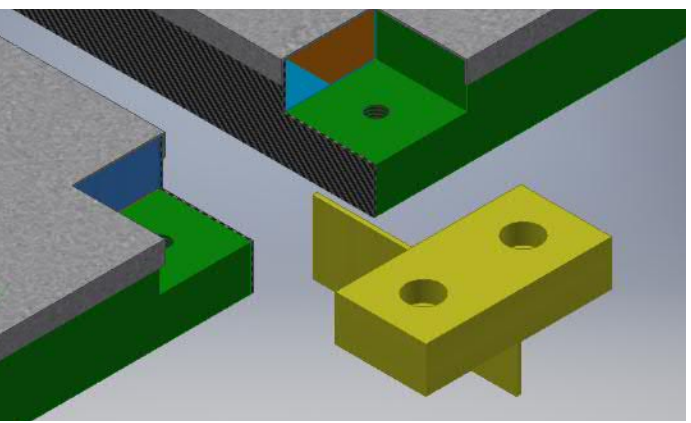

(d)

Figure 34. Improved version of the $28 \times 28$ cell CF tray. The tray is now subdivided in four smaller trays, and there is a further anchor point in the middle of each lateral section. From top left: the assembled tray (a), an exploded view (b), detail of the central tie (c), and detail of the lateral binding (d).

We have made various modifications, in order to increase the rigidity of the tray in the simulation. We have investigated the possibility of increasing the thickness of the base, of the lid (up to $1.5 \mathrm{~mm}$ ), we have also toyed with the idea of having two rigidly coupled trays, but even in this case, once the crystals are loaded, the resonant frequencies drops to roughly $30 \mathrm{~Hz}$. All these models were made extrapolating the parameters from the FEM model of the $6 x 6$ tray that was tested experimentally, as described in the previous sections. Given the results, we decided to change the design and to split the $28 \times 28$ tray into four $14 \times 14$ sub-trays, in an effort to increase the first mode resonance frequency. We further improved the $\mathrm{CF}$ tray structure robustness, by implementing an anchor at the centre with a thick CF tie (see figure 34). To study the stiffness of this consame figuration we implented using Ansys a FEM model of a panel the size of the $28 \times 28$ tray with the same overall thickness, with two skins of $0.5 \mathrm{~mm}$ thick carbon on the outside of an internal honeycomb core with the pattern of the crystal housings; to simulate the crystals we considered a distributed load of $165 \mathrm{~kg}$ total over the entire surface. We then divided this model into the 4 sub-parts and bound it at the centre and at the corners. With this solution, the first resonant 
frequency obtained (loaded tray) was $=125 \mathrm{~Hz}$ (see figure 35), so these simulations were then used as an initial input for the much more detailed simulations performed by LOSON that confirmed our results. The full sized CF trays were then built, following this updated design (see figure 36).
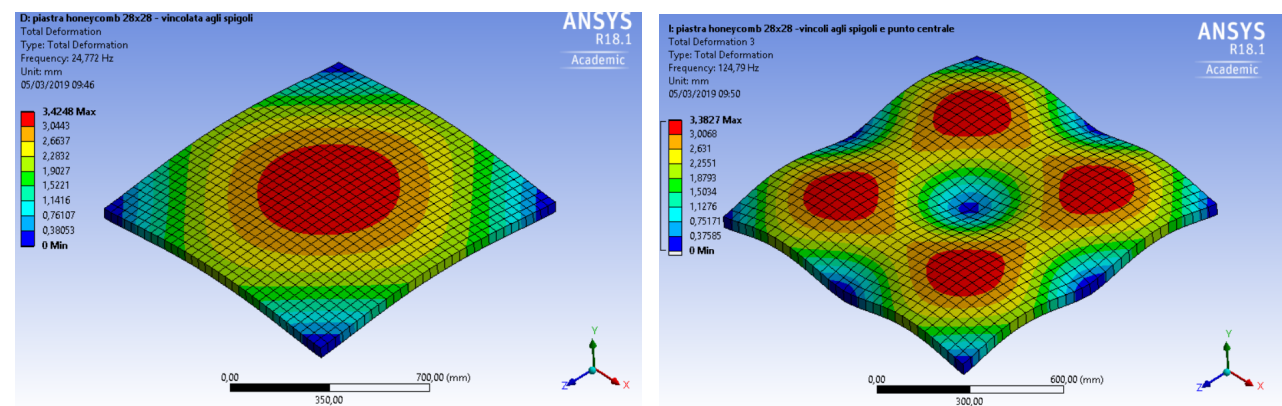

Figure 35. First mode resonant frequency of a simulated full sized $28 \times 28$ cell tray loaded with crystals. Before subdividing it into 4 sections ( $25 \mathrm{~Hz}$ resonance), after subdividision (resonance $125 \mathrm{~Hz}$ ).

There are four sub-trays with a pattern of $14 \times 14$ cells that can be joined in the centre with a CF tie and on the outer border of each frame. In the centre there is enough space to house 4 crystals with half the standard dimensions, minimizing the impact of the hole in the middle. The sub-trays

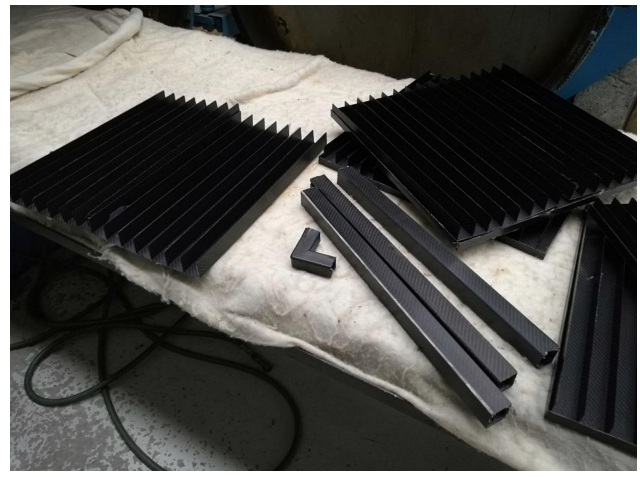

(a)

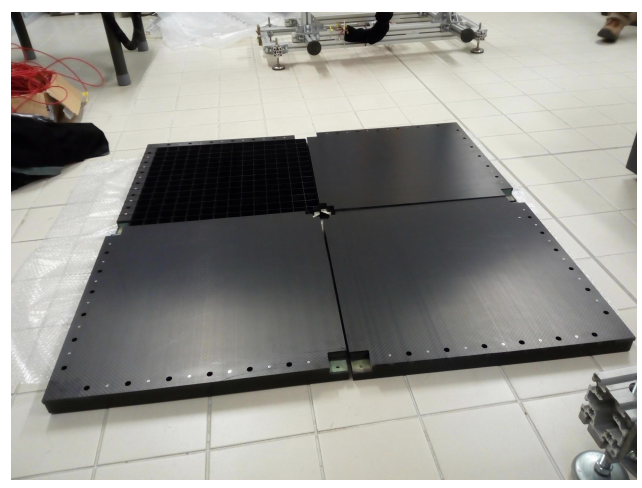

(c)

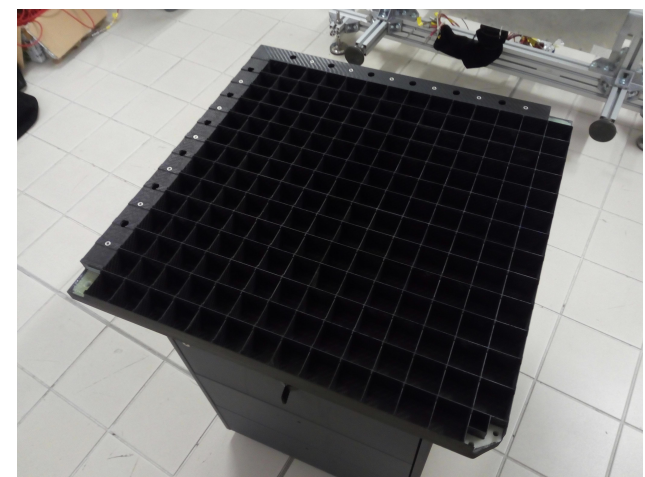

(b)

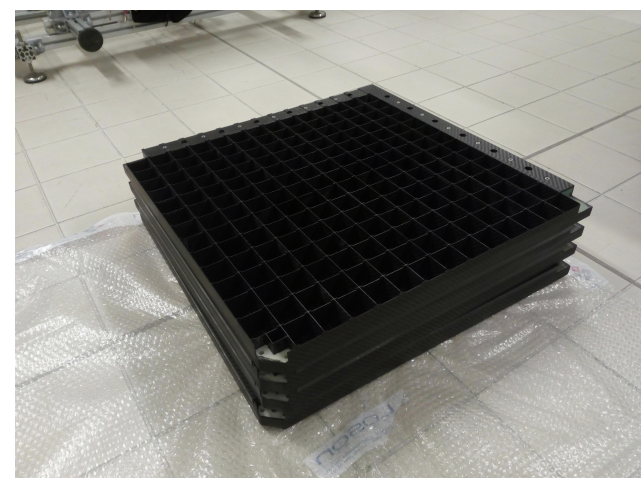

(d)

Figure 36. From top left: CF elements before the glueing procedure (a), a single sub-tray (b), full size tray with lid (c), and four stacked sub-trays (d). 
are independent and can even be stacked together. We have built a total of eight sub-trays with $14 \times 14$ cells that can also be stacked to form an eight layer calorimeter prototype that can be used at test beams.

\subsection{Calorimeter test beam prototype}

We have used the knowledge gained with all the measurments and studies performed on single crystals to build various calorimeter protoypes. The first prototype was built with only a few crystals of $\mathrm{CsI}(\mathrm{Tl})$ ( 20), coupled to large area VTH2090 PDs, with iron cubes used to fill the empty spaces without crystals, as shown in figure 37(a). These small trays were then piled together

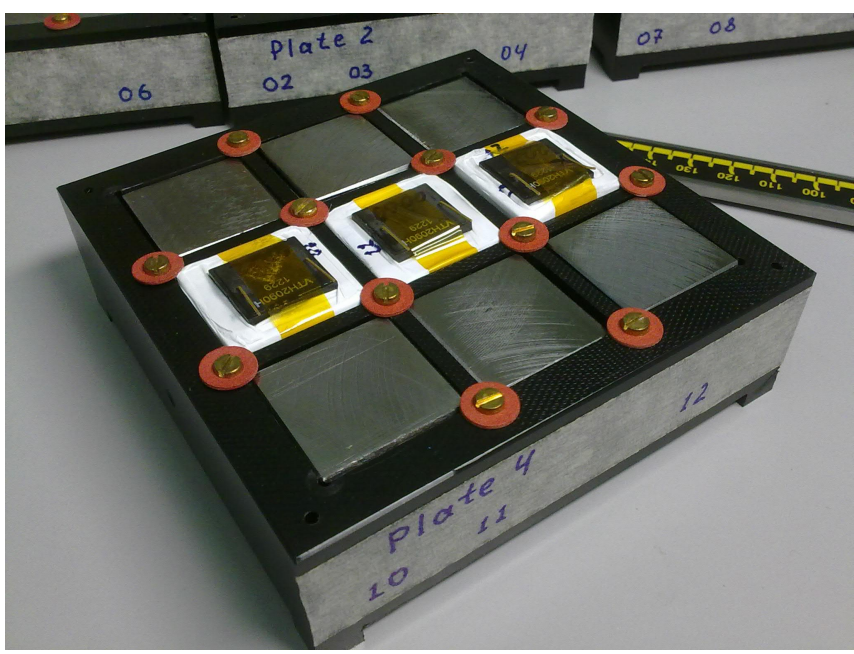

(a)

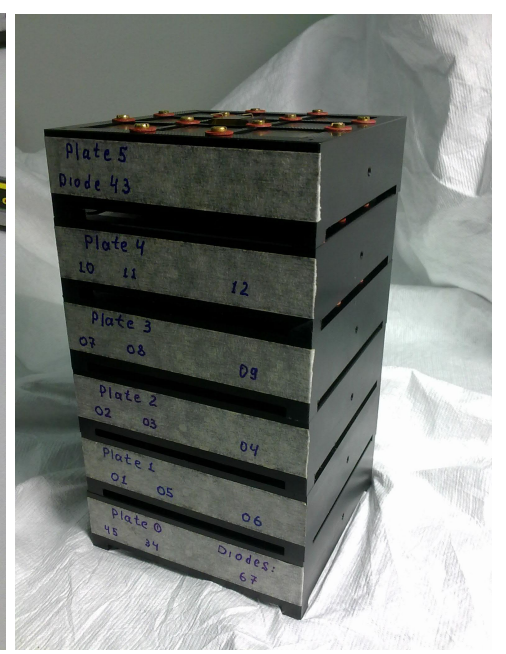

(b)

Figure 37. Photo of a small tray with $3 \mathrm{CsI}(\mathrm{Tl})$ crystals and iron cubes used as filler (a). Photo a the first small size calorimeter prototype used for our preliminary tests on elcromagnetic showers (b).

to form small calorimetric towers that we tested with high energy electron beams to study shower profiles (figure 37(b)). This first small size prototype was build to test the main ideas and basic characteristics of the calorimeter. The prototype was tested with $50 \mathrm{GeV}$ electrons and muons at Super Proton Synchrotron (CERN). Its $12 \mathrm{CsI}(\mathrm{Tl})$ crystals of $25 \tilde{\mathrm{A} U} 25 \tilde{\mathrm{A}} \mathrm{U} 25 \mathrm{~mm}^{3}$ were the first crystals acquired by our group. The size was chosen because we had some CsI(Tl) bars left from a previous experiment and they could be recycled using these dimensions. In fact, these first scintillators were cut and polished at our workshop in Florence (INFN). The mechanical support structure consisted of 6 plates with 9 elements in 3ÃU3 matrix, 54 elements in total. The gap between crystals was $3 \mathrm{~mm}$ in all directions. The PDs were read out by specially designed Front End Boards developed by us for this project. This paved the way for the full size prototype which we built once we optimised the scintillator material, size, wrapping, and PD layout.

The development of the Calocube mechanics continued then with a design used for a test beam prototype in 2012. To support the $\mathrm{CsI}(\mathrm{Tl})$ scintillator cubes $\left(36 \times 36 \times 36 \mathrm{~mm}^{3}\right)$ and the electronic read-out boards we chose a structure based on polyoxymethylene trays with a pattern of $6 \times 6$ cells, each cell with a transverse dimensions of $37 \times 37 \mathrm{~mm}^{2}$ ( $\mathrm{x}$ and $\mathrm{y}$ ), and a depth of $36 \mathrm{~mm}(\mathrm{z}$ ). The cell walls are $3 \mathrm{~mm}$ thick, resulting in a $40 \mathrm{~mm}$ pitch in the $\mathrm{x}, \mathrm{y}$ directions for the crystals (see figure 38). 
These trays have been used for all tests performed by our collaboration both at test beam facilities

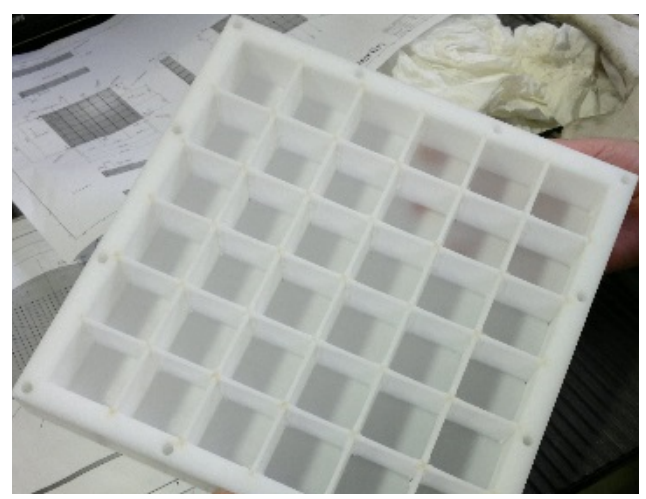

(a)

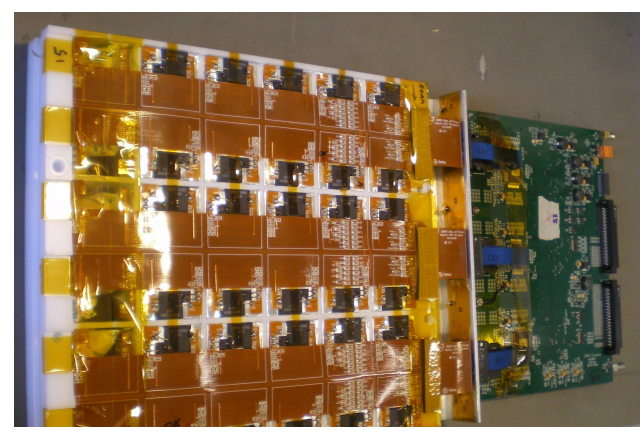

(c)

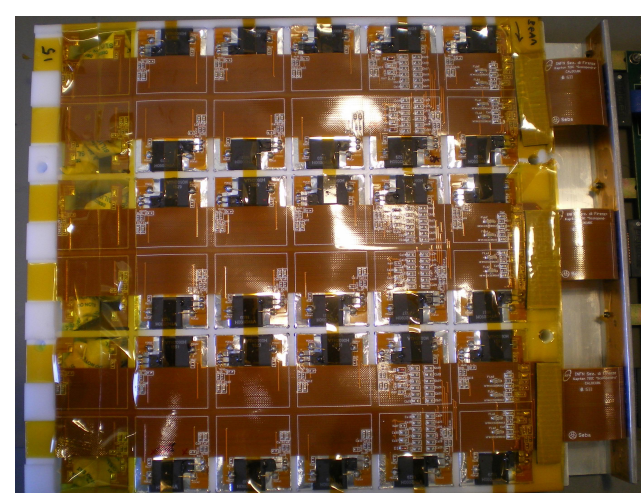

(b)

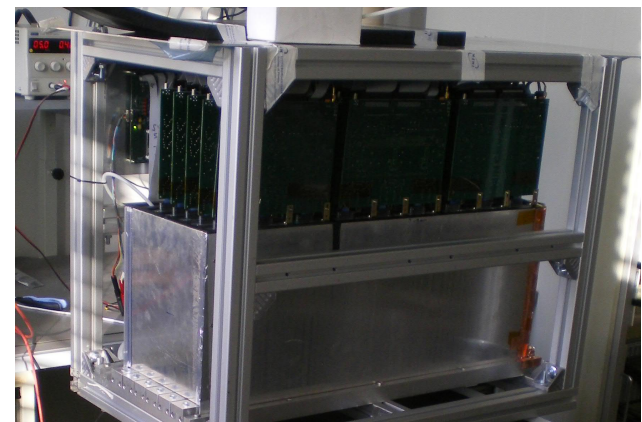

(d)

Figure 38. From top left: One of the first polyoxymethylene trays developed for the Calocube prototype (a), loaded with CsI (Tl) crystals and with the aluminium $\mathrm{C}$ section bolted at the end (b), with the F.E. boards fixed to the $\mathrm{C}$ section (c), fully assembled prototype calorimeter (d). Also visible are the kapton cables used to route the photodiodes signals to the F.E. boards

and in our laboratories. Each tray has a $\mathrm{C}$ section in alumium attached to the end that allows us to interconnect the crystals inside with the prototype Front End electronic boards (F.E.) developed by our collaboration in Trieste [40, 41]. Fifteen loaded trays have been stacked together, with a $4 \mathrm{~mm}$ spacing inbetween trays in order to have a $40 \mathrm{~mm}$ pitch also in the $\mathrm{z}$ direction. This assembly is what constitutes our Calocube calorimeter prototype that has been subject to many test at various beam facilities, (see bottom of figure 38 where a photo of a fully assembled tray with its F.E. and of the complete calorimeter protype are shown). A total of 25 trays were produced at the INFN Pisa workshop using a CNC milling machine from a single $40 \mathrm{~mm}$ thick plate.

In order to experiment with different crystal assemblies of varying sizes (i.e. CsI(Tl) $36 \times 36 \mathrm{~mm}^{2}$, LYSO 30x30 $\mathrm{mm}^{2}$ ), we have developed a new design based on a honeycomb plastic matrix suitable for 3D printing (figure 39). This will be our baseline for future tests of new calorimeter designs. The honeycomb structure allows us to achieve a very high rigidity while keeping the weight of the tray and plastic consumption at a minimum. The printing takes only a couple of hours and it is relatively easy to change cell dimensions to accomodate different crystal sizes. 

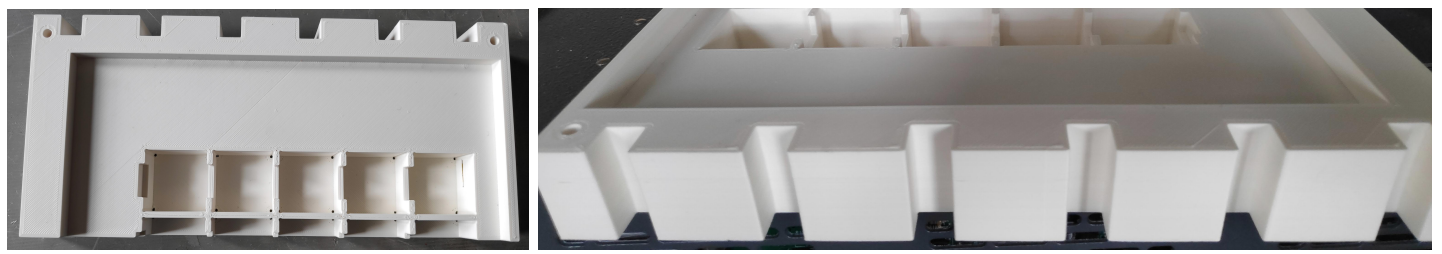

Figure 39. The first 3D printed tray protoype developed for future Calocube crystal tests with different scintillator materials (i.e. LYSO). The tray is nearly $40 \mathrm{~mm}$ thick.

\section{Front End electronics and final Test Beam prototype}

While the A250 by Amptek provides exceptional performance in terms of signal to noise ratio, it remains a very expensive single channel device with a limited dynamic range. For our calorimeter $R \& D$ we pursued the development of a dedicated very low power consumption Front End amplifier.

\subsection{The CASIS Front End Electronics}

The CASIS (CAlorimetry in SIlicon for the Space ) chip [40, 44] is an Application Specific Integrated Circuit (ASIC) specifically designed for space calorimetry by the INFN section of Trieste. For this scope the chip has been designed with a high dynamic range ( $\sim 50 \mathrm{pC}$ maximum input charge with a ENC of 2000-3000 electrons), that allows to study the interactions of high energy particles, keeping a low power consumption (2-3 $\mathrm{mW}$ per channel). The chip consists of 16 front-end channels with

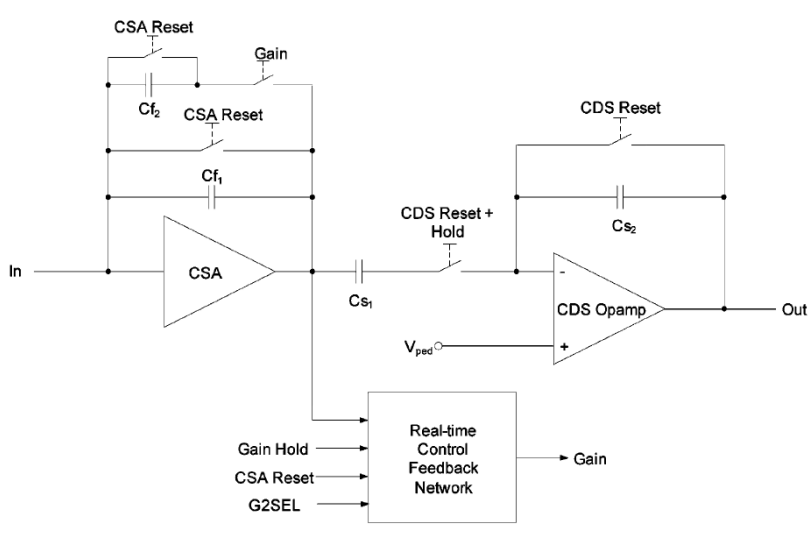

(a)

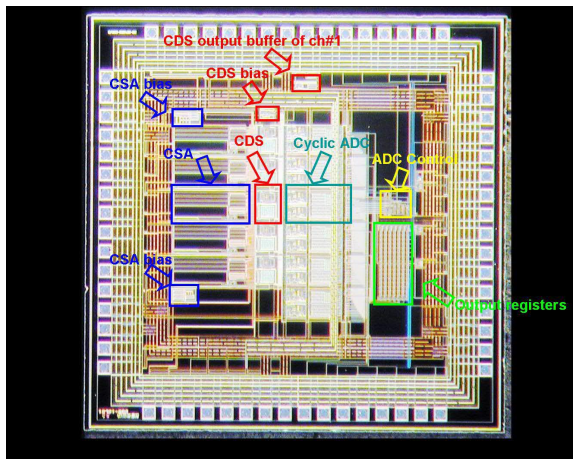

(b)

Figure 40. Circuit block diagram of the Front End amplifier (a) and silicon layout of a complete 16 channel chip (b).

double correlated sampling and one multiplexed ADC. The control circuitry uses external clock, convert and reset lines to generate all the required signals to operate the sampler and then the ADC. The chip has been designed and realized with the 0.35 Âtm C35B4 CMOS technology of Austria Micro Systems [45], which provides two polysilicon and four metal layers, with a power supply voltage of $3.3 \mathrm{~V}$.

The schematic block diagram of the front end section is shown in figure 40(a). A charge sensitive amplifier (CSA) is followed by a correlated double sampling filter (CDS). To achieve the very large 
required dynamic rage, a new architecture of the CSA feedback was implemented. It consists of a double gain loop with a real-time selection circuitry: a $1.6 \mathrm{pF}$ capacitor is permanently connected to a folded cascode amplifier which sets the high gain, while a second larger capacitor ( $30.4 \mathrm{pF})$ is ready to be automatically inserted in parallel whenever the input signal exceeds a given threshold. A switch is used to reset periodically the preamplifier. To minimize the power consumption and guarantee a fast response, the comparator of the feedback control circuit is implemented with a Schmidt trigger with a value of the threshold of about $2 \mathrm{pC}$. Since the charge integrated on the small capacitor is not destroyed when the system switches to low gain, a precise calibration of the total range can always be performed.

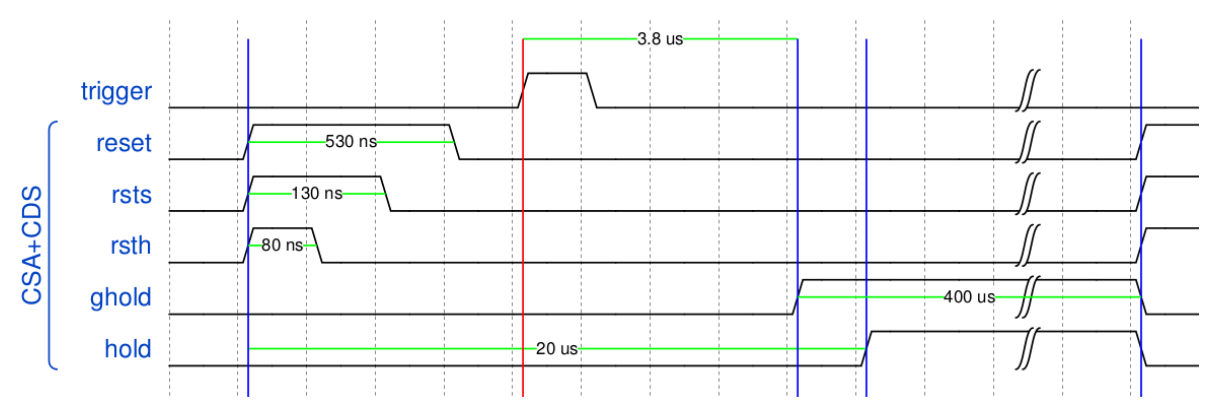

Figure 41. Timing sequences for the CSA and CDS blocks.

The voltage swing at the CSA output is about $1.5 \mathrm{~V}$ and the nominal sensitivities for the high and low gains are $0.67 \mathrm{mV} / \mathrm{fC}$ and $0.03 \mathrm{mV} / \mathrm{fC}$, corresponding respectively to roughly $50 \mathrm{pC}$ of maximum charge on the input before saturation sets in. This large range coupled to the previously mentioned low power consumption, is one of the main benefits of the switched integrating capacitor design. This feature coupled to the dual PD readout (large area and small area) ensures the $10^{7}$ dynamic range that we aimed for in the Calocube project. The CSA output is sampled by the CDS

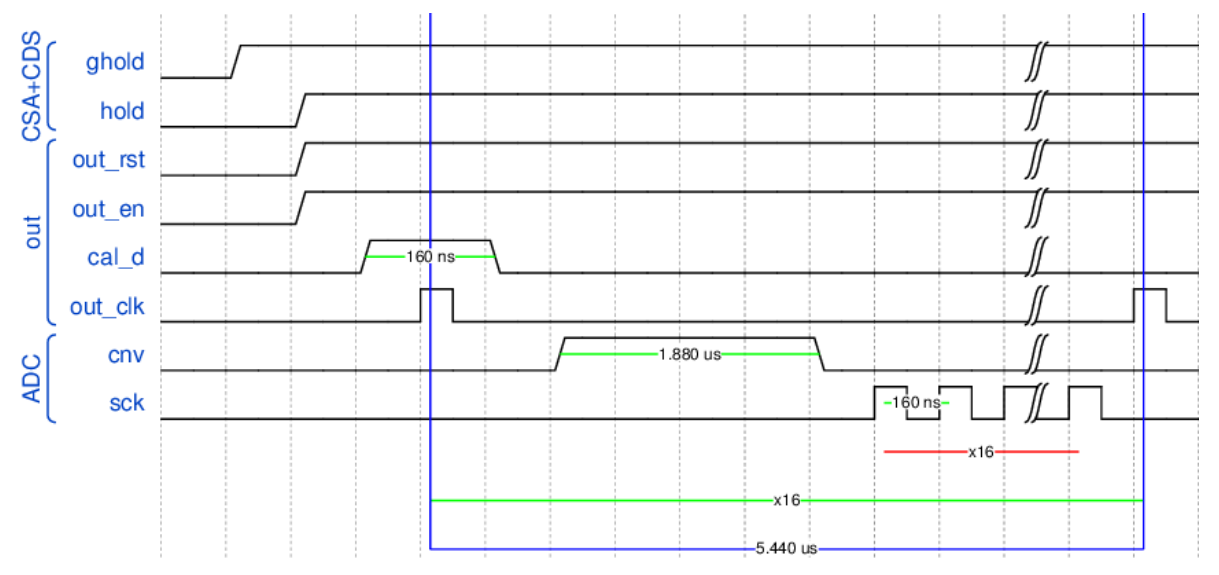

Figure 42. Timing sequences fot the Hold and ADC part.

filter. This element executes a baseline substraction, using two samples for each event. The first one acquired before the trigger (periodically on every reset pulse falling edge) and the second one with the trigger itself. When a trigger is present, the reset is inhibited, a HOLD signal is generated after 
a fixed (adjustable) delay, and the CSA outputs the difference between the baseline and the trigger events. The DC pedestal of the CDS output (normally set at $900 \mathrm{mV}$ ) can be adjusted externally by means of the Vped bias. The timing sequences are shown in figure 41 .

The signals for the CSA+CDS control are: RST H, RST S, RESET, GHOLD, and HOLD. The first tree signals perform a periodic reset of both the CSA (RST H, RST S) and of the CDS (RESET). In fact the chip must be reset periodically to remove the charge accumulated on the capacitors. All three signals are synchronized on the rising edge whereas their width are determined by the CASIS setup times. The HOLD and GHOLD lock the sampled voltage and automatic gain switch, respectively, before clocking the ouptut and ADC circuitry. The chip has one analogue output that provides a multiplexed output for all 16 channels. This multiplexing operation is driven by the the OUT CLK and the OUT RST lines. Thus it is possible to connect to the output all 16 channels, one by one, with 16 clock pulses. The OUT EN should be high to enable chip output.

The complete diagram of the output control signals is shown in figure 42. The SCK (ADC clock) width is $80 \mathrm{~ns}$ with a period of 5.44 Âţs and drives the conversion of each single output. These values were chosen on the basis of the ADC timing characteristics. More details are available both in [44] and in [19].

\subsection{The calorimeter assemblies}

For the first prototypes we designed and built a few boards using the CASIS chips (see figure 43(a)) which were coupled to the PDs through custom built flexible PCBs designed by us. A first version of these circuits are shown in figure 43(b)). Once decided to opt for CsI(Tl) $36 \times 36 \times 36 \mathrm{~mm}^{3}$ crystals,

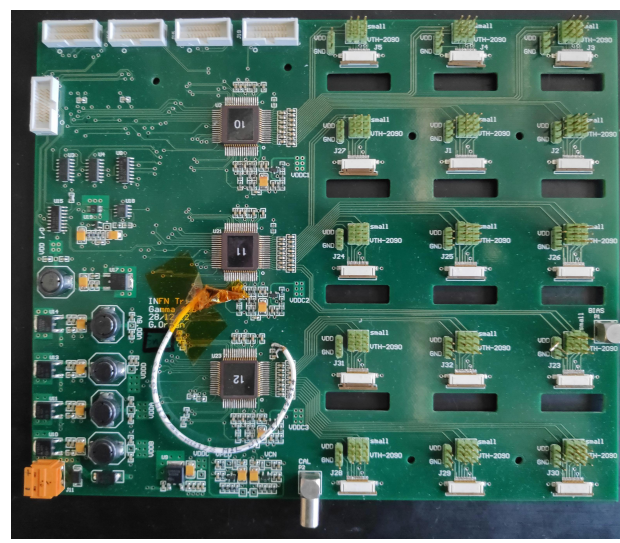

(a)

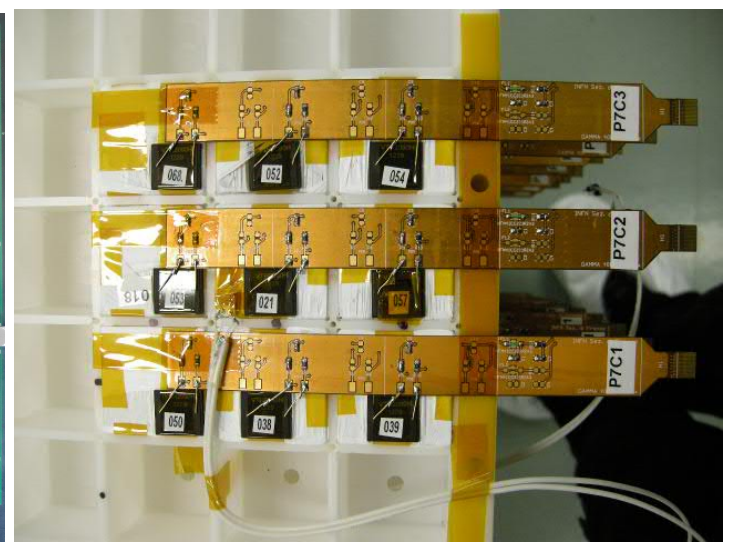

(b)

Figure 43. Photo of a CASIS board with three chips (a). The board was designed to allow the connection of the chips to the PDs of the scintillator crystals with flexible circuits (see text), which were threaded through holes in the PCB itself. Front view of a prototype assembly with an early version $(3+3$ PDs) of the flexible circuits in full view with their tails ready for insertion (b).

we used the polyoxymethylene trays described in section 3.3 for the prototype mechanics. The crystals, wrapped in Vikuiti, are loaded inside the trays with their PDs glued to them.

The connection to the F.E. electronics is realised with flexible PCBs, which provide not only a shielded connection from the PDs to the F.E. electronics, but have also on board components for 
the biasing and decoupling of the High Voltage used by the diodes. A partial circuit diagram that exemplifies this is shown in figure 44(a). The circuit referes to our latest implementation with each diode being biased through a $10 \mathrm{~K} \Omega$ resistor with a $100 \mathrm{nF}$ blocking capacitor placed in proximity of the PD itself. Two common biasing section are shared between 12+12 diodes, one for the large area and one for the small area ones. The anode signal from the diodes is sent to the F.E. electronics. The common reference is not ground but the $3.3 \mathrm{~V}$ power plane. This is due to internal details of the chip and is the configuration that minimises common mode noise $(\mathrm{CMN})$ pick up. The component layout for our final version is shown in figure 44(b) using a $10+10$ design, where we kept two channels of the F.E. electronics connected to normal diodes soldered on the flex circuit, used exclusively for CMN subtraction. The total measured ADC value can be written as:

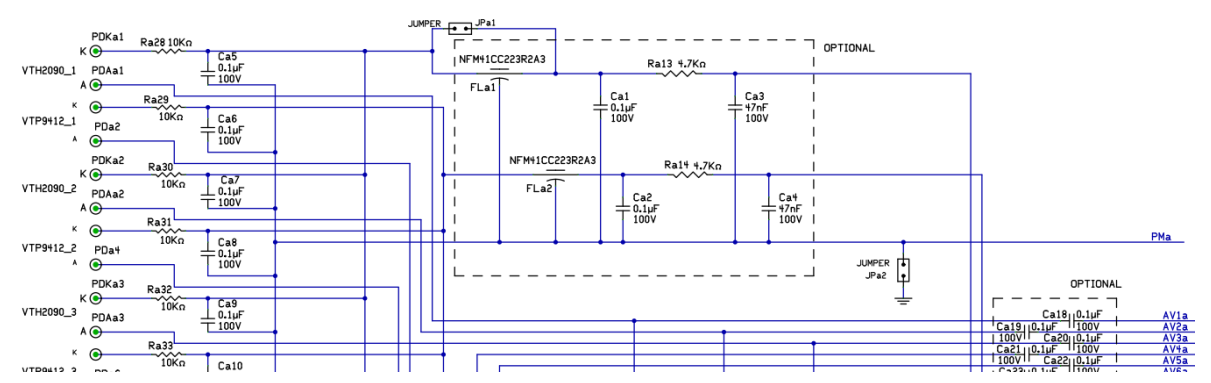

(a)

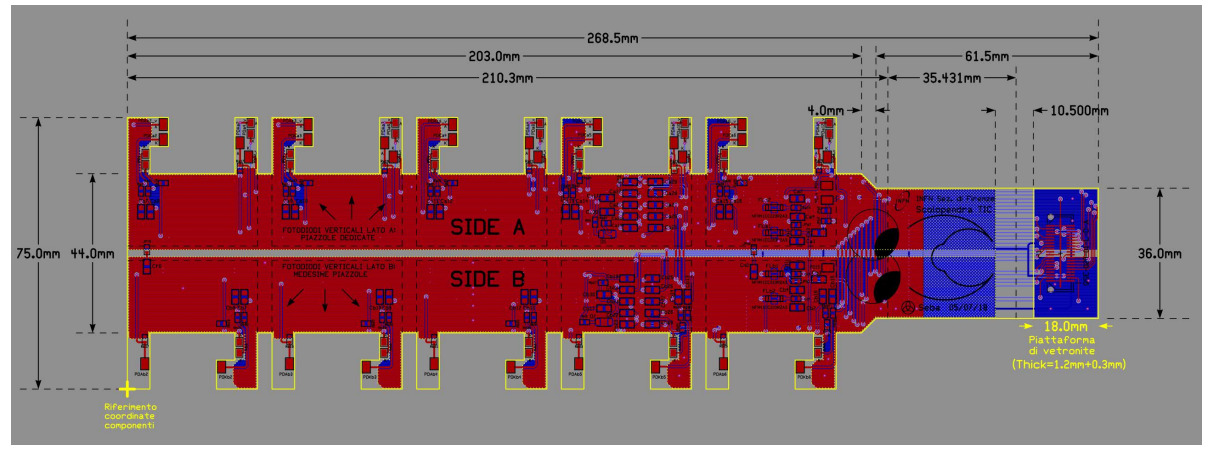

(b)

Figure 44. Partial schematic of the flexible PD circuits (top). PCB layout of the components (below)

$$
A D C_{i}^{j}=S_{i}^{j}+P E D_{i}+C N^{j}
$$

where $i$ is the number of the CASIS cannel $(i=0, \ldots, 15), j$ is the event number $(j=0, \ldots, N$, where $N$ is a total number of acquired events), $S_{i}^{j}$ is a physical signal of the interacting particle, $P E D_{i}$ is the pedestal of the channel $i$ and $C N^{j}$ is the common noise corresponding to the event $j$. Usually $C N^{j}$ is calculated by using channels of the chip with no signal present (i.e. not invested by a shower signal). In a calorimeter this is not always the case and it's the reason we have sacrificed at least one channel of each chip (even in the latest designs) to sample the $C N^{j}$ value. Figure 45 shows the significant improvement obtained by subtracting the CMN on a typical CASIS channel pedestal distribution. 


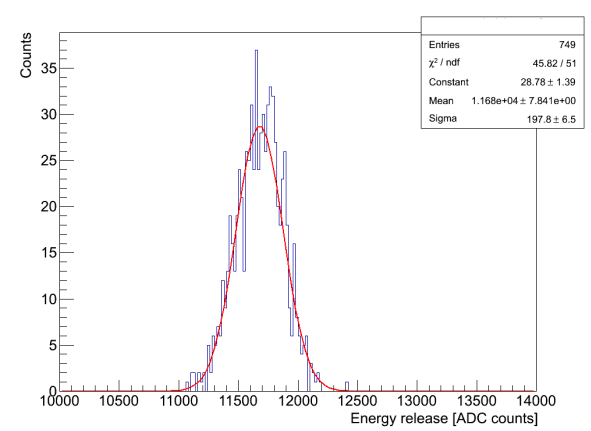

(a)

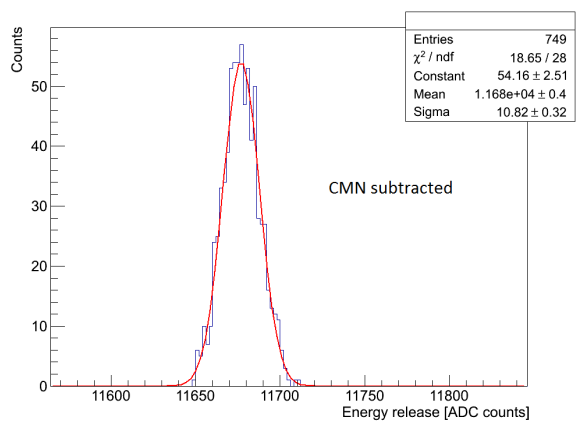

(b)

Figure 45. Pedestal distribution for a typical CASIS channel connected to a PD. Before (a) and after CMN subtraction (b). The horizontal scales are different for the two plots. For comparison, with this setup, a MIP signal peaks at around 180 AC channels.

Since a MIP signal in our CsI(Tl) peaks at roughly 180 ADC channels (see figure 46) this translates into an increase in the signal to noise ratio for MIPs, from 1 to roughly 18, which is more than enough to allow the use of MIPs for calibration purposes. This result also testifies to the excellent dynamic range achieved with this proptotype design and electronics. In fact, by changing CASIS settings and integration time sequences, the actual MIP signal can vary even by $50 \%$, while the signal to noise ratio remains more or less constant. A further improvement was obtained by using the Vikuiti wrapping (as stated previously) with increases in the signal up to $40 \%$.

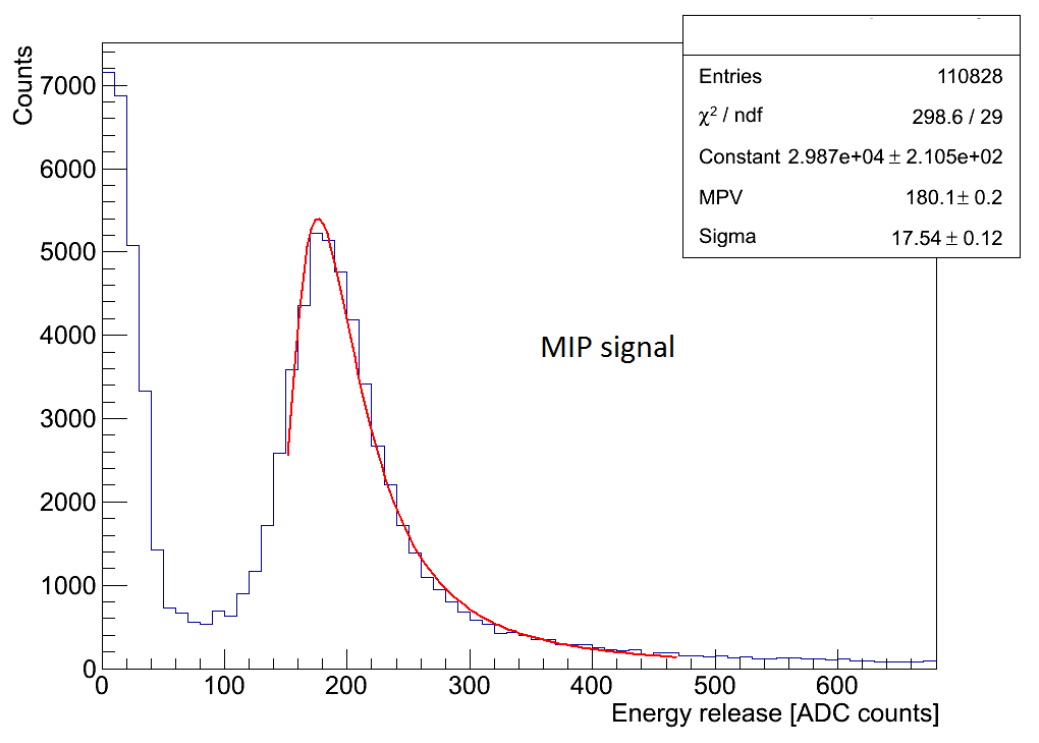

Figure 46. Cosmic ray spectra for one of the crystals inside a tray read out with the CASIS board after pedestal and CMN subtraction. 


\subsection{Prototype Assembly and Readout}

We began a first prototype construction by assembling 14 trays each one with a $3 \times 3$ crystal matrix (see figure 47(a)), for a total of 126 crystals. We used a total of three CASIS boards for a total

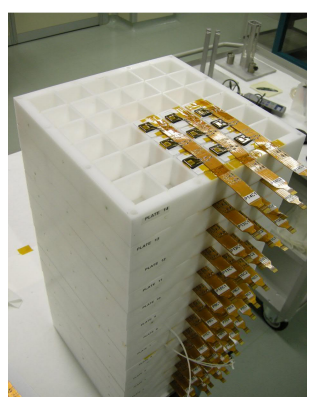

(a)

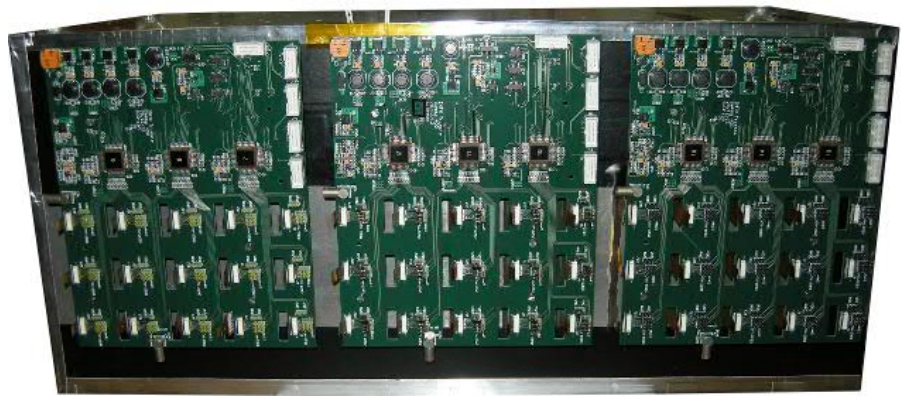

(b)

Figure 47. The stack of trays each with 9 crystals (a). The assembled calorimter (126 crystals) read out by three CASIS boards placed on top of the stacked trays (b).

of nine Front End chips to readout the crystals. The whole was placed in an aluminium box that provided electrical and light shielding from the outside (see figure 47(b).

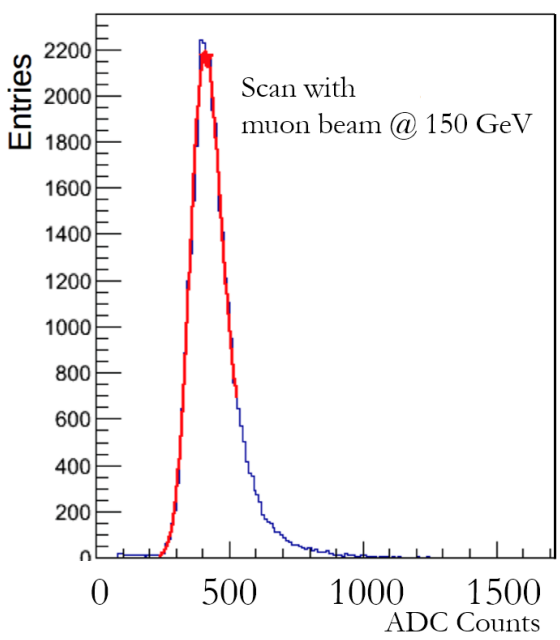

(a)

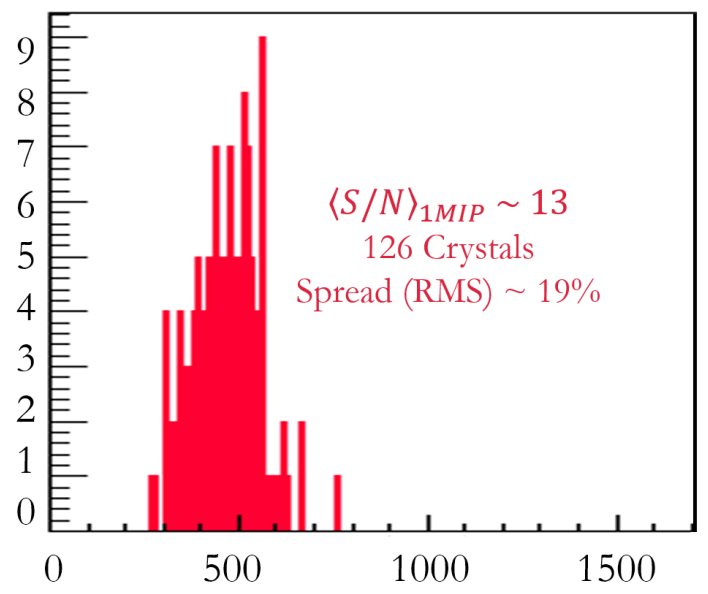

(b)

Figure 48. Typical crystal respons to a $150 \mathrm{GeV}$ muon beam (a). The histogram (b) shows the ditribution of the peak values of the 126 Landau fits.

This first step was used by us to validate the whole assembly procedure and the PDs coupling to the electronics. We checked the response to MIPs with the CASIS Front End (the analogue part has been kept in all subsequent developments, i.e. like the HIDRA chip mentioned later), and the uniformity of response of the wrapped crystals. Figure 48(a) shows the excellent S/N ratio we have managed to obtain for MIPs, without sacrificing the very high dynamic range of the calorimeter, proving that MIPs can be easily identified and used for calibration. Figure 48(b) shows the most 
probable values of the fitted Landau of each of the 126 crystals used in the first assembly. The dispersion is contsined within $19 \%$ showing excellent reproducibility of the wrapping and optical coupling of the crystal to the PDs.

The F.E. electronics was driven by a back end board, the Read Out Controller (ROC) developed by us as a generic board for DAQ applications. This board is based on a Xilinx Virtex 4 FPGA that can be programmed to provide the relevant signals needed by the F.E. and to transfer tha acquired data via fast USB (QuickUSB) or Ethernet links. The board of which a photo is shown in figure 49
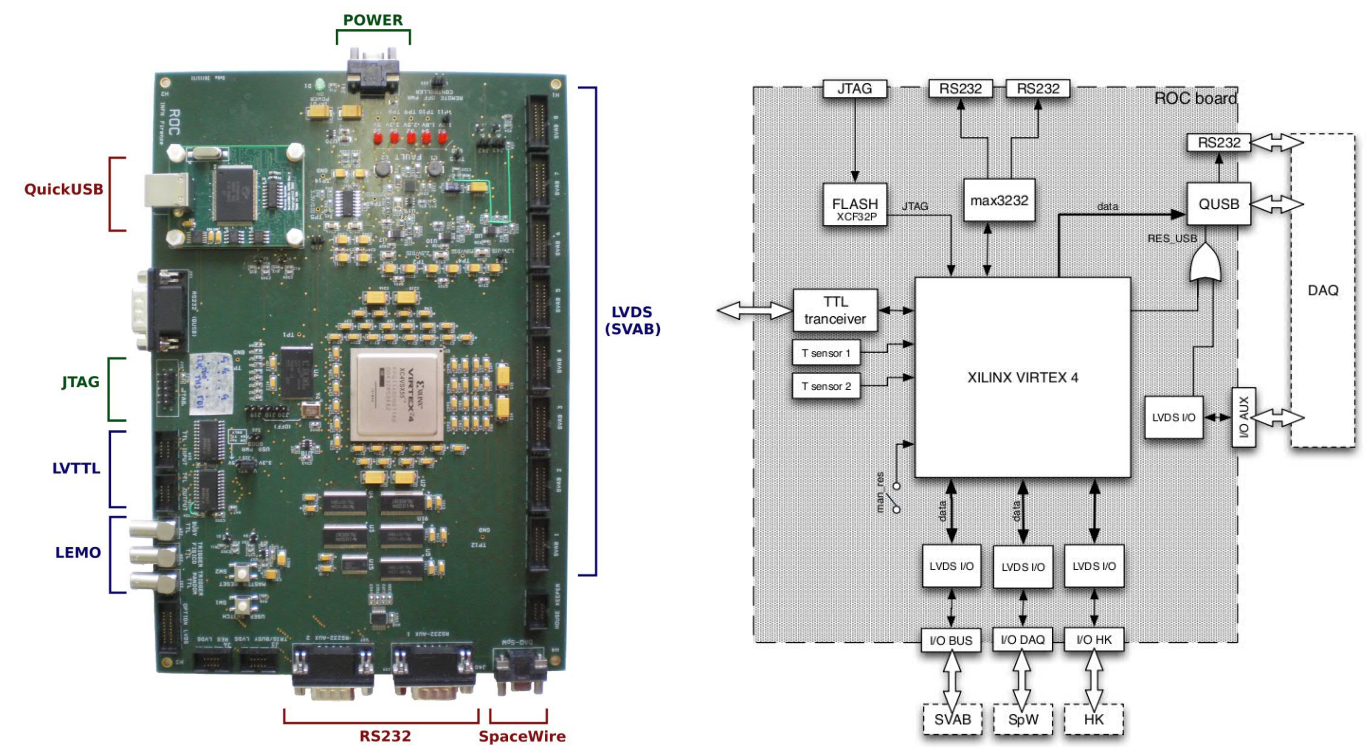

Figure 49. Back end board (ROC) used for readout (left). Block diagram of the ROC (right).

has many LVDS I/O which we used to pilot the CASIS chips while we used a Quick USB piggy back to transfer the data from the CASIS ADCs to an external computer and storage. This board has been used by us for all the data taking and with all the Calocube prototypes including the final one with the HIDRA chips (an evolution of the CASIS design, next section), highlighting its extreme versatility. More information on the ROC board and the Finite State Machine used to drive the CASIS chips can be found in [46].

Finally we show (figure 50(a), etc.) the imaging properties of the calorimeter highlighting some reconstructed events from the various test beams performed at SPS-CERN with Calocube. The pictures underline the ease with which particle discrimination can be performed. The detailed lateral and longitudinal profiles obtained with this detector allow us also to compensate for the energy leakage present in hadronic showers. All figures show the deposited energy for every crystal, with a longitudinal energy profile segmented layer by layer (14 in total, corresponding to $27 \mathrm{X}_{0}$ and $1.3 \lambda_{I}$ ). The beam is aligned with the centre cube. The first figure (50(a)) shows the profile of a muon (a MIP) that produces a relatively low signal in all the centre cubes and only in them. The energy release is more or less the same from one layer to the other, with the small residual fluctuations due to the Landau process and differences in response for the different channels of the chips. Events of this type are in fact chosen for calibration purposes usually by fitting the Landau peak. The next figure (50(b)) shows an event where a MIP (a proton) interacts in the centre 


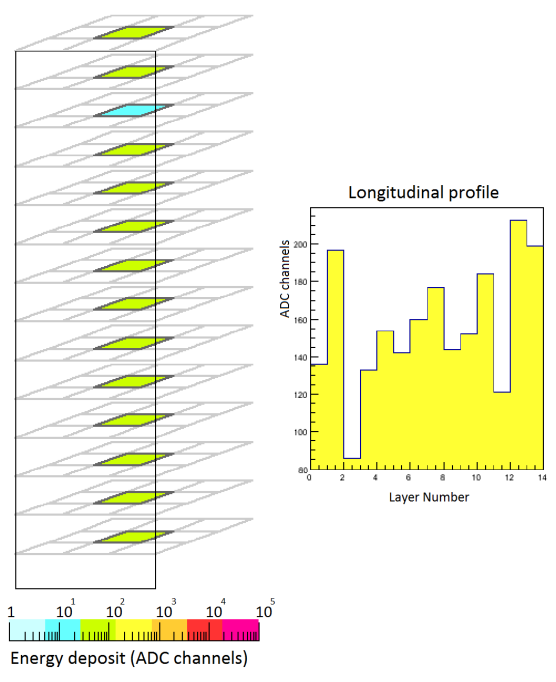

(a)

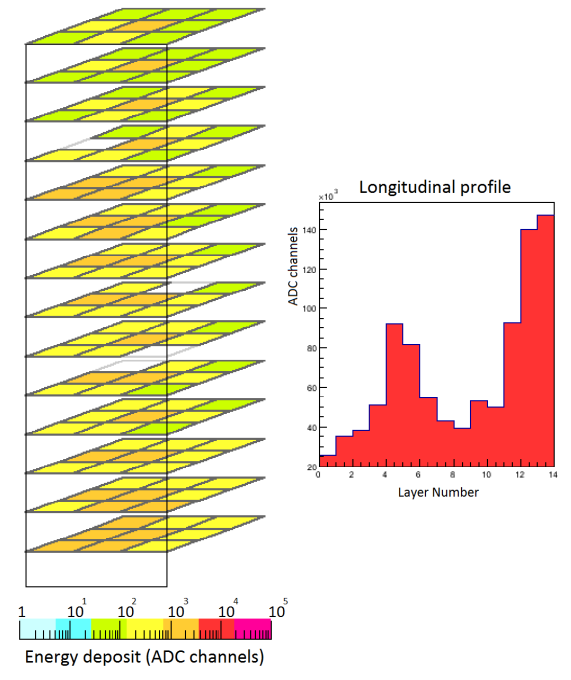

(b)

Figure 50. Graphic visualisation of the reconstructed events in the Calocube prototype. A muon (a) and a proton (b). See text for details.

cube of layer 4, inititating a shower in the next layers. The shower develops rapidly and releases a significant amount of energy in the second part of the calorimeter. The interesting fact is that there is a very visible, high energy release aligned with the centre cubes in layers 4 to 7 . This behaviour is compatible with a formation, within the hadronic shower, of an electromagnetic shower caused by a $\pi^{0}$ decay. The next two images (figures 51(a) and 51(b) ) are of two particles that interact

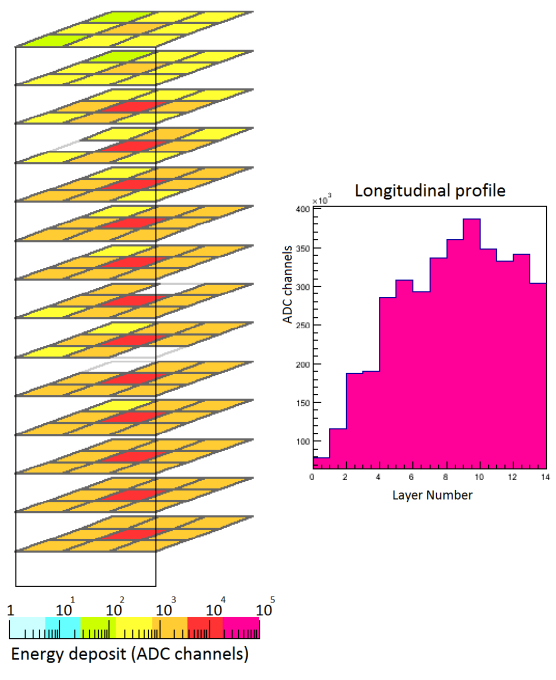

(a)

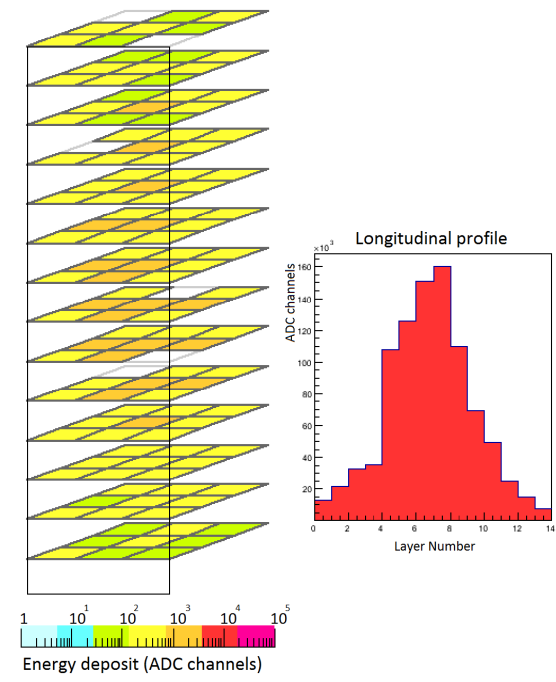

(b)

Figure 51. Graphic visualisation of the reconstructed events in the Calocube prototype. A proton (a) and an electron (b). See text for details. 
straight away in the first layer. The first one has a lateral profile typical of a hadronic shower. The shower development is relatively slow and reaches the maximum between layers 9 and 10, where the maximum deposited energy is observed. An electromagnetic component is distinguishable from the significant energy release in the central row of the crystals between layers 2 and 14. The last one is an electron shower profile that is fully contained.

\subsection{Current and final development}

The CASIS chip itself has now evolved in a new version called HIDRA. The analogue part is the same but we have further improved its behaviour and have added self triggering capabilities and logical output registers for the trigger logic. With this new chip we have realised new boards (see figure 52) and flexible connection circuits for our final Calocube prototype. The final prototype
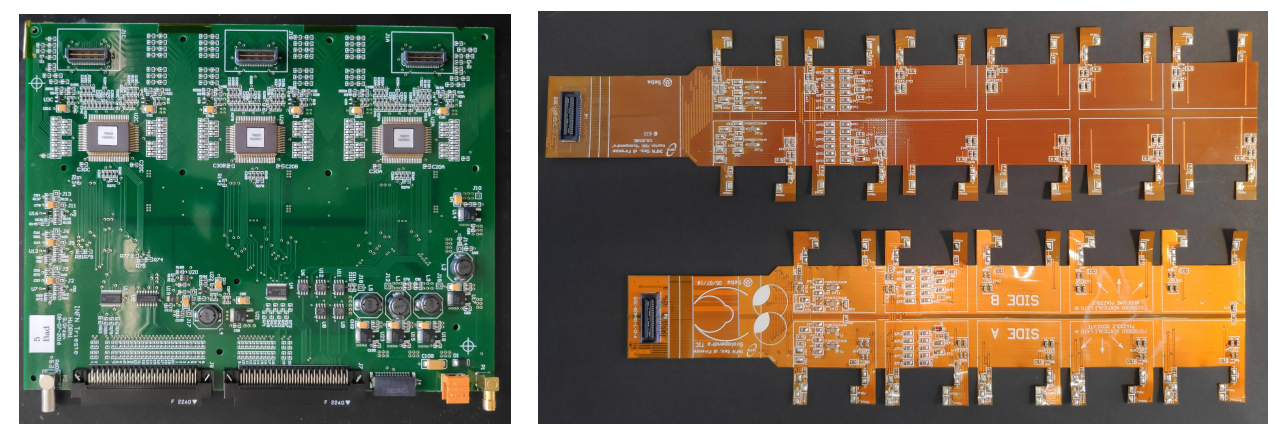

Figure 52. The new HIDRA board (left) and the flexible circuits that connect the crystals to it (right). Each tray as explained in section 3.3 has bolted on an aluminum section to which a HIDRA board is fixed.

design uses the same mechanical trays but has a more modular approach with one F.E. board serving only one tray. This allows easier intervention on the prototype whenever repairs or substitutions need to be done on the crystals in the trays. This is exemplified by the photo of a fully equipped

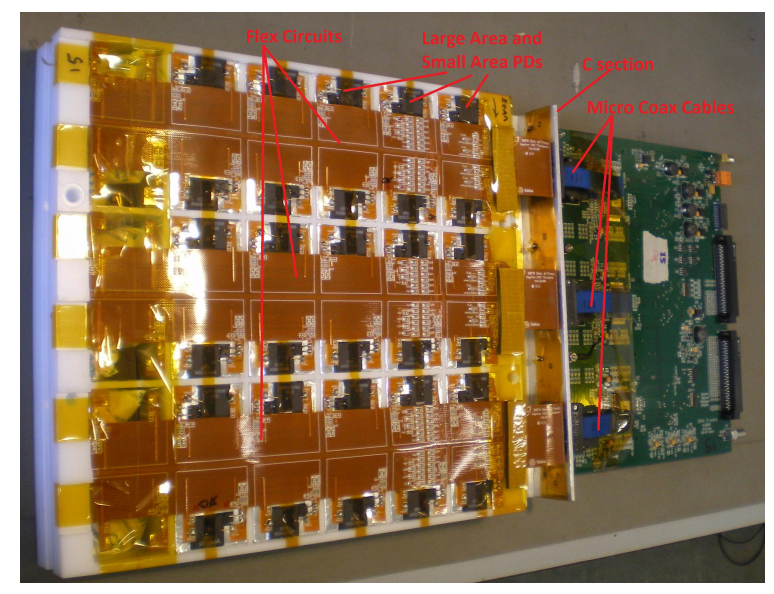

Figure 53. The current tray assembly. A C section aluminium piece holds the HIDRA board to the tray. Final connections between the Flex circuits and the F.E. board are realised with SAMTEK microcoax cables.

tray shown in figure 53. The 18 fully equipped trays (36 crystals each) for a total of $648 \mathrm{CsI}(\mathrm{Tl})$ 
crystals constitute the larger Calocube prototype with which data has been taken with protons, pions, electrons, muons, and above all ions at various test beam facilities. The Calocube prototype (figure 54) even though finished is still an object of R\& D which we modify accordingly to test new ideas or concepts. Currently we are modifying the front part to make place for a few layers of silicon tracking modules to test a gamma ray application evolution (TIC, Tracking In Calorimeter [47]) that would still be capable of performing cosmic ray physics in orbit mantaining the highest performance for charged particles, while allowing accurate directional gamma ray physics down to $1 \mathrm{GeV}$.

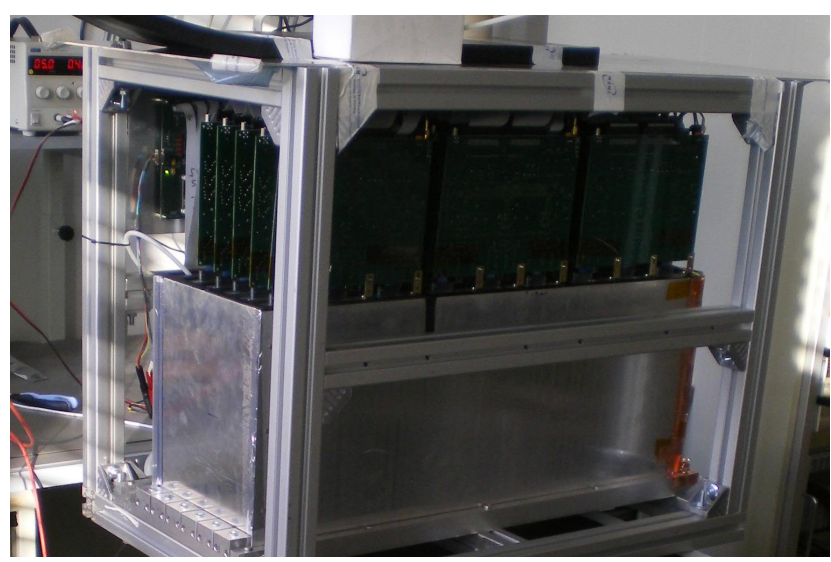

(a)

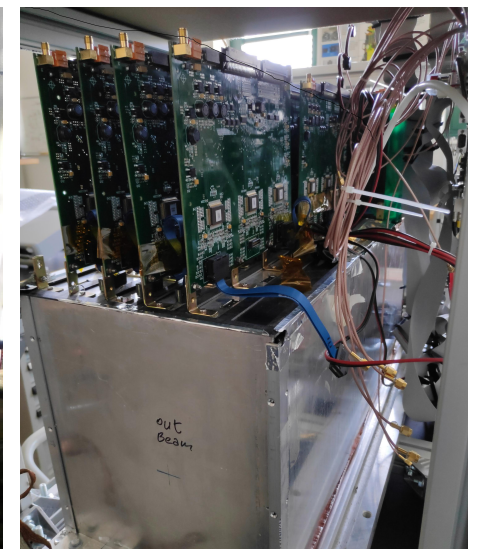

(b)

Figure 54. The final assembled Calocube prototype with the latest F.E. boards (a). A detail showing an intervention taking place and the modular structure of the final prototype (b).

\subsection{Dual readout studies}

Although as stated previously, detailed results from the various test beams we performed belong to a future paper, we end this section reporting on a very interesting calorimetry technique that we have tested with $\mathrm{CsI}(\mathrm{Tl})$ crystals during our prototype development. This is a self contained project within our R\&D that has shown promise but is currently too expensive to pursue further.

One of the main causes of the hadron energy measurement low resolution, are the fluctuations in the fraction of E.M. showers within the hadronic shower. This fraction not only fluctuates but also depends on the energy of the incident hadron. A long standing effort by the DREAM collaboration [48, 49] has shown that a Dual Readout paradigm, where both scintillation and Cherenkov light are read out thorough two independent channels, can be used to correct the calorimeter response on an event basis. This can bring about a dramatic improvement in resolution at the price of a more complex readout scheme. Basically while the scintillation light is sensitive to both hadronic and E.M. shower components, Cherenkov radiation is mainly produced in E.M. showers. Figure 55(a) shows the correlation between the two signals in simulations of $100 \mathrm{GeV}$ and $500 \mathrm{GeV}$ protons. When both signals are available, the overall signal fluctuation (Cherenkov vs Scintillation, given by the projection on the line of slope R, see figure) is much reduced respect to fluctuations present in each signal.

We performed various tests to see if we could tease out the Cherenkov signal from $\mathrm{CsI}(\mathrm{Tl})$ scintillator. Due to the physics process underlying the two light production mechanisms, there 
are important differences in the spectra and time development of the two components that can be exploited to measure each one separately. In fact Cherenkov photons are produced concurrently with the particle passage through the crystal and as such last only a ns or less, also Cherenkov light peaks at short wave lengths in the ultraviolet region and can be easily separated from the green peaking scintillation one. We tested these assumptions with a special setup shown in figure 55(b), where a scintillator bar (CsI(Tl) is read out at its ends with two fast, UV sensitive phototubes. Since

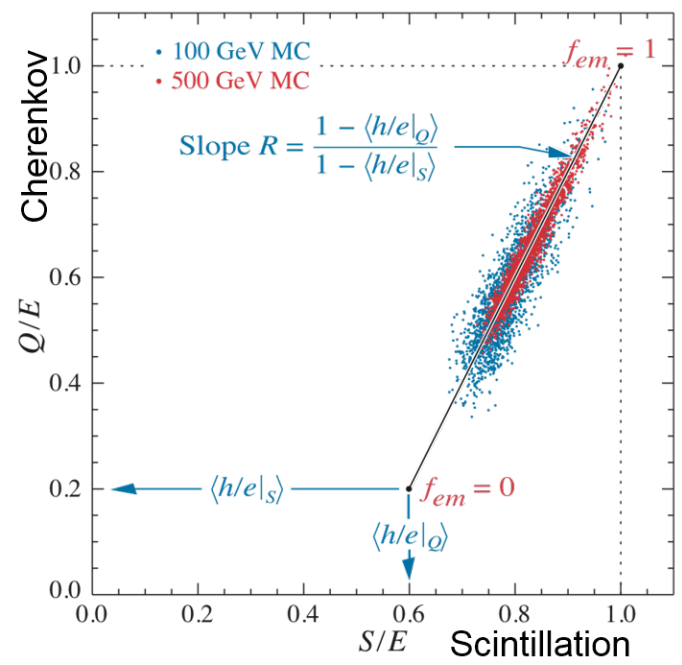

(a)

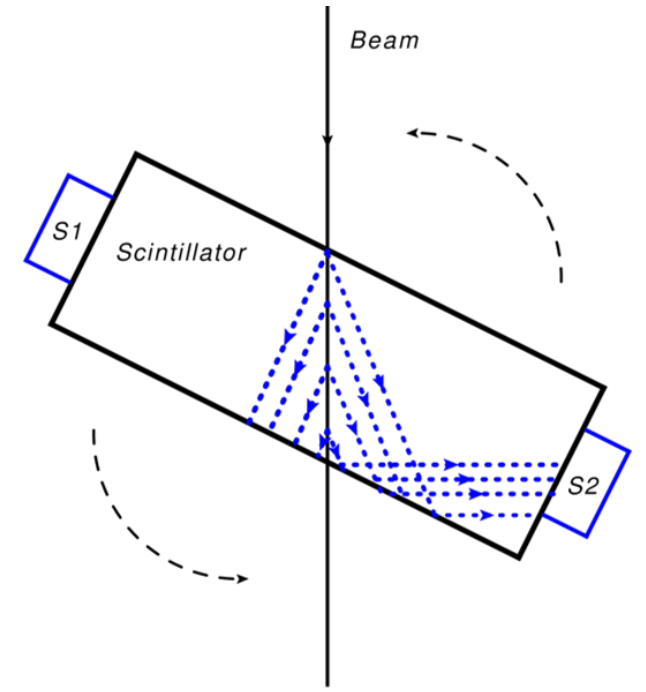

(b)

Figure 55. Simulations of hadronic showers at two different energies. The figure (a) shows the scintillation signal vs Cherenkov signal scatter plot. The extra information provided by the Cherenkov light helps reducing overall fluctuations in the calorimeter signal. Schematic of the scintillator test setup, with a PM at each end for readout, showing the principle of operation for Cherenkov light separation (b).

Cherenkov is directional along the particle path, turning the crystal respect to the beam favours one PM respect to the other. Scintillation light on the other hand is isotropic and as such no dependence on the angle should be seen on the two PMs. As shown in figure 56 we have managed to find clear evidence for Cherenkov light production in CsI(Tl) crystals. The Cherenkov signal is much higher when the crystal is rotated at 30 degrees and is evident only in the first few ns. Unfortunately, even though $\mathrm{CsI}(\mathrm{Tl})$ scintillation light has a time constant of 600ns, its output is so abundant that it anyway swamps the Cherenkov photons. Using interference filters, we have managed to block the visible light (i.e. scintillation) leaving only the UV component. Figure 56(b) shows how the signal behaves exactly as a directional Cherenkok light would behave when the crystal is rotated.

As a final remark on Dual Readout, we would like to point out that while the technique is very promising for a full sized calorimeter, we have shown (with simulations) in [12] that the relative improvement in energy resolution that can be expected for a typical CaloCube depth of $2 \lambda_{I}$ is only $10 \%$. This is to be compared to a $30 \%$ or more improvement that can be obtained for a full containment calorimeter. The reason for such a difference, is to be found in the large longitudinal leakage and its relative fluctuations which become very significant for a thin calorimeter and that dominate the energy resolution performance. 


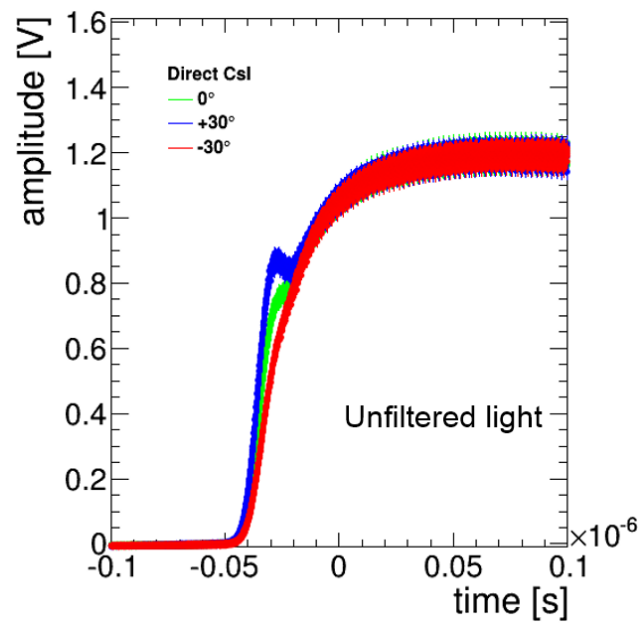

(a)

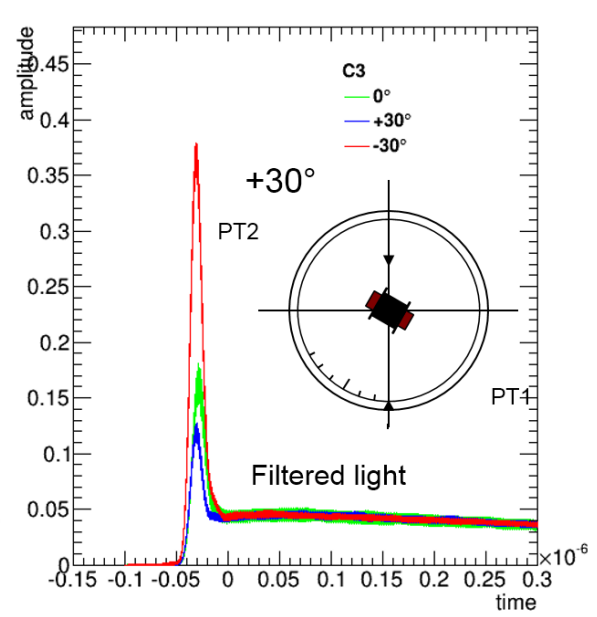

(b)

Figure 56. CsI(tl) signals acquired with a fast digitizer (a). The three angles refer to the crystal rotation respect to the beam axis that can increase or decrease the Cherenkov light on the PM. Cherenkov light is evident in the first few ns but is anyway swamped by the much more abundant scintillation component. If a filter blocking visible light is applied in front of the PM the Cherenkov component becomes prominent (b).

\section{Conclusions}

The Calocube project has lasted more than four years. During this period, we designed and constructed a prototype calorimeter to study its performance in view of a possible future space experiment. We have demonstrated that a highly segmented calorimeter design can be built using inorganic crystal scintillators and a photodiode readout. Our high segmentation design allows for excellent particle ID and energy resolution, while increasing significantly the geometrical acceptance of the detector.

The project is now evolving both as a full scale space proposal (HERD), and as a test bench to experiment novel ideas for space based cosmic ray detectors (LAPUTA and TIC projects financed by INFN). The test beam data has been shown at various meetings and conferences and will be published in the near future by our collaboration.

\section{Acknowledgments}

We wish to acknowledge the financial support of INFN, in the framework of the CSN5 2013 Calls, that funded the Calocube project.

\section{References}

[1] Antoni et al. , Astropart.Phys.24:1-25,2005

[2] Amenomori et al. , Adv.Sp.Res.47:629-639,2011

[3] Bartoli et al. , arXiv:1401.6987 (to be pub. Chinese Phys. C)

[4] Bartoli et al. , arXiv:1502.03164 
[5] ICRC2011, Beijing, Measurements of the Longitudinal Development of Air Showers with the P. Auger Observatory, P. Auger Collaboration.

[6] HiRes Collaboration, PRL 104 (2010) 161101

[7] ICRC2013, Rio De Janeiro, Progress towards understanding the analyses of mass composition made by the Auger and Telescope Array Coll.

[8] ICRC2005, Pune, Chemical composition of ultra-high energy cosmic rays estimated by muon measurement with AGASA

[9] M. Tanabashi et al. (Particle Data Group), Phys. Rev. D 98, 030001 (2018)

[10] O. Adriani et. al., "Development of a 3-D cubic crystal calorimeter for space: CaloCube", proceedings of the 1st Conference on Calorimetry for the High Energy Frontier (CHEF 2013), Paris, 2013

[11] http://herd.ihep.ac.cn/

[12] O. Adriani et al., CaloCube: An isotropic spaceborne calorimeter for high-energy cosmic rays. Optimization of the detector performance for protons and nuclei, Astropart.Phys. 96 (2017) 11-17, DOI: 10.1016/j.astropartphys.2017.10.002

[13] Richard Wigmans 2012 J. Phys.: Conf. Ser. 40401206

[14] E. Vannuccini et al. , Nucl.Instrum.Meth. A845 (2017) 421-424

[15] O. Adriani et al. , Nucl.Instrum.Meth. A845 (2017) 439-442

[16] https://amptek.com/products/a250-charge-sensitive-preamplifier

[17] https://www.excelitas.com/product-category/photodiodes-and-arrays

[18] http://amptek.com/products/px5_digital_pulse_processor

[19] Starodubtsev O., Calocube development of a homogeneous, isotropic and large dynamic range calorimeter for the study of primary cosmic rays in space experiments, Ph.D. thesis; supervisor: Raffaello D’Alessandro ; tutor: Pier Simone Marrocchesi. [Siena, 2015]. FIS/01, BNI 2015-257T

[20] G. Giacomini, L. Bosisio, I. Rashevskaya, O. Starodubtsev. Noise Characterization of Double-Sided Silicon Microstrip Detectors With Punch-Through Biasing, IEEE Transactions on Nuclear Science IEEE TRANS NUCL SCI , vol. 58, no. 2, pp.569-576, 2011

[21] F. Scholze et al., Nucl. Instrum. Methods A439, 208 (2000)

[22] http://www.crystals.saint-gobain.com/CsI(Tl) scintillator.aspx

[23] http://www.crystals.saint-gobain.com/BGO Scintillator.aspx

[24] http://www.crystals.saint-gobain.com/PreLude 420 Scintillator.aspx

[25] Rihua Mao et al., Emission spectra of LSO and LYSO crystals excited by UV light, X-ray and $\gamma$-ray , IEEE Transactions on Nuclear Science 55(3):1759 - 1766, July 2008

[26] http://www.omegapiezo.com/crystal scintillators.html

[27] Junwei Du et. al. Physical Properties of LYSO Scintillator for NN-PET Detectors. Biomedical Engineering and Informatics, 2009. BMEI âĂŹ09. 2nd International Conference on. 2009 / IEEE / 978-1-4244-4132-7

[28] http://www.nist.gov/pml/data/star/ 
[29] ICRU Stopping power and ranges for protons and alpha particles (Report 49) http://www.icru.org/home/reports/stopping-power-and-ranges-for-protons-andalpha-particles-report49

[30] M. Thiel, W. M. Doring, V. Dormenev, P. Drexler, R. W. Novotny, M. Rost, and A. Thomas High-Energy Photon Detection With LYSO Crystals IEEE TRANS-ACTIONS ON NUCLEAR SCIENCE, VOL. 55, N. 3, JUNE 2008, 1425

[31] G.M. Onyshchenko et al. Comparative light yield measurements of oxide and alkali halide scintillators, Nuclear Instruments and Methods in Physics Research A 537 (2005) 394396

[32] Y. K.Gupta et. al. Characteristics of CsI(Tl) crystal coupled with PIN photodiode for $\gamma$-rays and charged particles. Proceedings of the International Symposium on Nuclear Physics (2009) pp.654

[33] Y. Larochelle et al., Nucl. Instr. and Meth. in Phys. Res. A 348 (1994) 167

[34] F. Aharonian et al. (HESS Collab.), Phys. Rev. Lett. 101, 261104 (2008) and arXiv:0905.0105

[35] http://multimedia.3m.com/mws/media/374730O/vikuiti-tm-esr-salesliterature.pdf?fn=ESR\%20ss2.pdf

[36] Hiroyuki Ishibashi, Seikichi Akiyama, Mitsuru Ishii, Effect of Surface Roughness and Crystal Shape on Performance of Bismuth Germanate Scintillators, Ibaraki Research Laboratory, Hitachi Chemical Co., Ltd., 1380 Tarasaki Katsuta, Ibaraki 312

[37] Glenn F. Knoll, Radiation Detection and Measurements, (2010) page 235, John Wiley \& Sons, Inc.

[38] Loson s.r.l., Milan, Italy, https://loson.it/

[39] SERM s.r.l., Terni, Italy, https://www.sermssrl.com/

[40] V. Bonvicini et al., CASIS1.1: a very high dynamic range front- end electronics with integrated Cyclic ADC for calorimetry applications, IEEE Nuclear Science Symposium, Vol. 2, pg.1078 - 1081, (2007)

[41] V. Bonvicini et al., A Double-Gain, Large Dynamic Range Front-end ASIC With A/D Conversion for Silicon Detectors Read-Out, IEEE Trans. Nucl. Sci., Vol. 57, No. 5 (2010)

[42] Marrocchesi et al. , Nucl.Instrum.Meth. A692 (2012) 240-245

[43] Marrocchesi et al. , Nucl.Instrum.Meth. A692 (2012) 240-245

[44] V. Bonvicini et al. A Double-Gain, Large Dynamic Range Front-end ASIC With A/D Conversion for Silicon Detectors Read-Out. Nuclear Science, IEEE Transactions on, 57(5):2963-2970, 2010

[45] http://www.austriamicrosystems.com/

[46] Berti E., Developement and first tests of a calorimeter prototype for the measurement of cosmic rays in orbit: Calocube, Master Thesis; supervisor: R. D’Alessandro, University of Florence (2012)

[47] E. Berti et al., CaloCube: a new concept calorimeter for the detection of high energy cosmic rays in space, Journal of Physics: Conference Series, 1162, (2019), doi:10.1088/1742-6596/1162/1/012042

[48] N. Akchurin, et al., Dual-Readout calorimetry with crystal calorimeters, Nucl.Instrum. and Meth., A598 (2009) 710âĂŞ721. doi:10.1016/j.nima.2008.10.010.

[49] N. Akchurin, et al., Separation of crystal signals into scintillation and Cherenkov components, Nucl. Instrum. and Meth., A595 (2) (2008) 359 âĂŞ 374, doi:http://dx.doi.org/10.1016/j.nima.2008.07.136. 